\title{
MILITARY ECOLOGICAL RISK ASSESSMENT FRAMEWORK (MERAF) FOR ASSESSMENT OF RISKS OF MILITARY TRAINING AND TESTING TO NATURAL RESOURCES
}

January 2002

Glenn W. Suter II

Keturah A. Reinbold

Winifred H. Rose

Manroop K. Chawla

Environmental Sciences Division

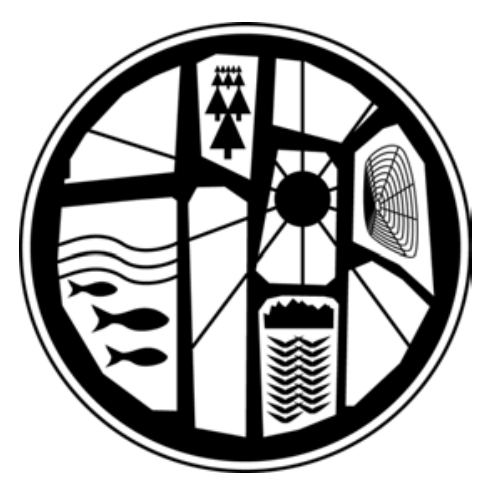





\title{
MILITARY ECOLOGICAL RISK ASSESSMENT FRAMEWORK (MERAF) FOR ASSESSMENT OF RISKS OF MILITARY TRAINING AND TESTING TO NATURAL RESOURCES
}

\author{
Glenn W. Suter II \\ Environmental Sciences Division \\ Oak Ridge National Laboratory \\ (currently at National Center for Environmental Assessment, USEPA)
}

\section{Keturah A. Reinbold \\ Winifred H. Rose \\ Manroop K. Chawla}

U.S. Army Construction Engineering Research Laboratory

January 2002

Prepared for

U.S. Department of Defense

Strategic Research and Development Program

\author{
Prepared by \\ OAK RIDGE NATIONAL LABORATORY
}

Oak Ridge, Tennessee 37831

managed by

UT-BATTELLE, LLC

for the

U.S. DEPARTMENT OF ENERGY

under contract DE-AC05-00OR22725

and

U.S. Army Construction Engineering Research Laboratory

Champaign, IL 61826 


\section{TABLE OF CONTENTS}

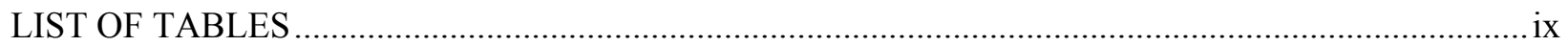

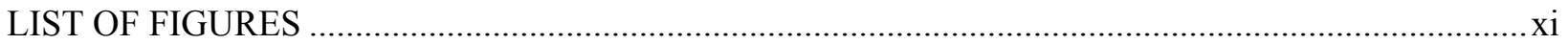

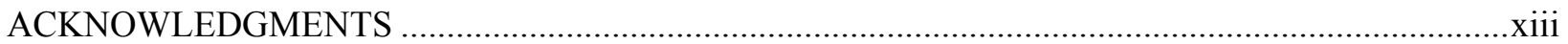

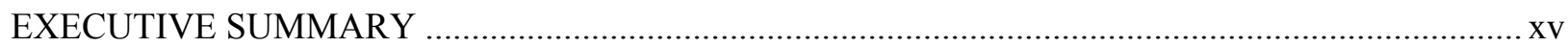

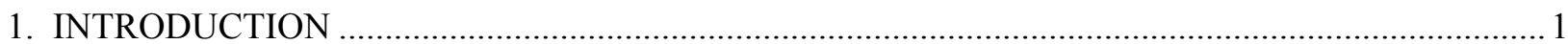

1.1 BACKGROUND AND OBJECTIVE

1.1.1 Background......................................................................................................

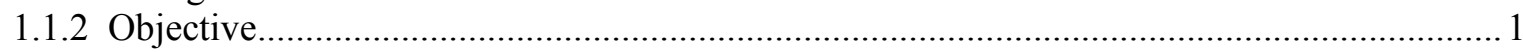

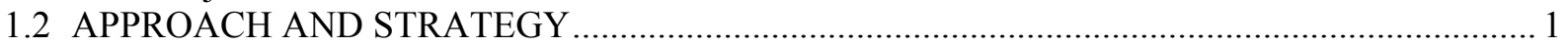

1.2.1 Adaptation for Place-Based and Programmatic Assessments ..............................................2

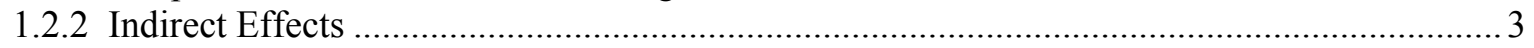

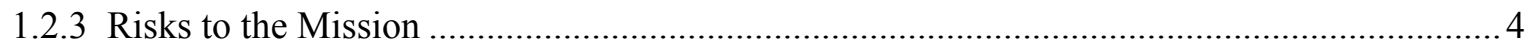

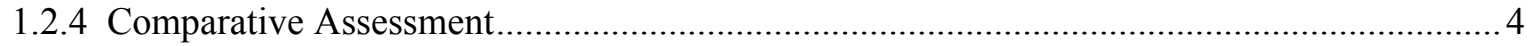

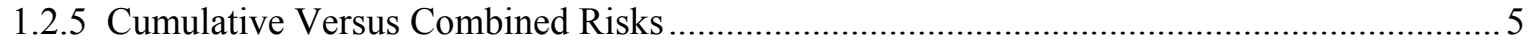

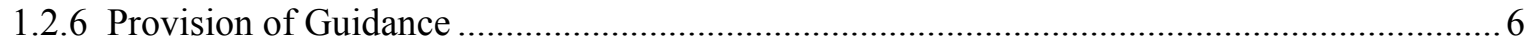

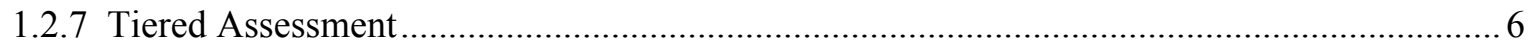

1.2.8 Geographic Information Systems …………………….............................................

1.3 ORGANIZATION OF THE RESEARCH ……………….................................................

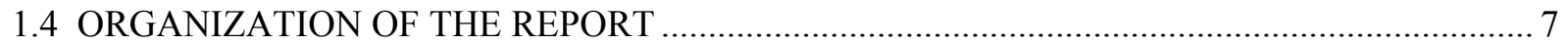

2. RISK MANAGER AND RISK ASSESSOR ............................................................................ 11

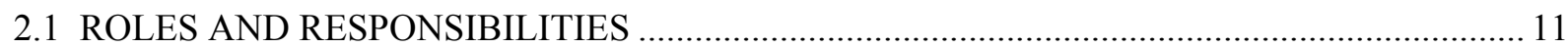

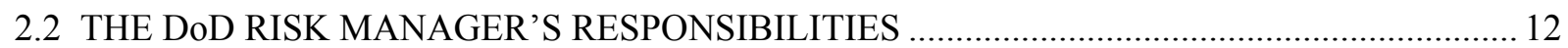

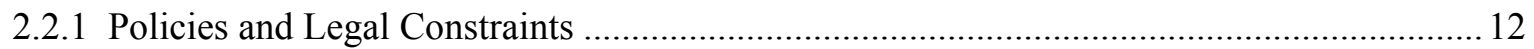

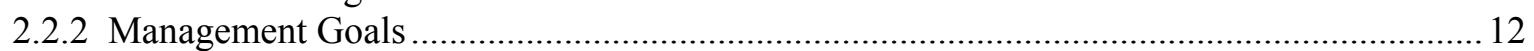

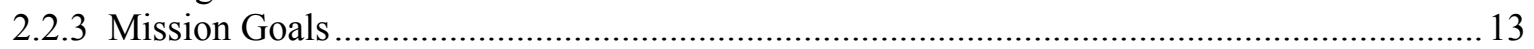

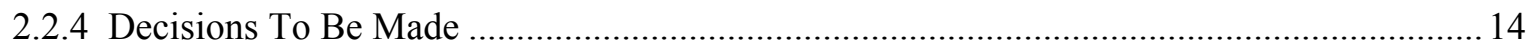

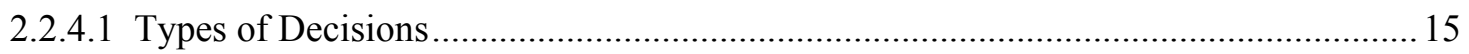

2.2.4.1.1 Comparative Decisions................................................................................ 15

2.2.4.1.2 Noncomparative Decisions........................................................................... 15

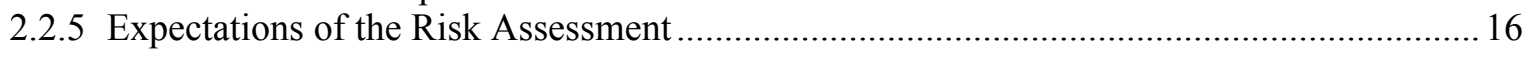

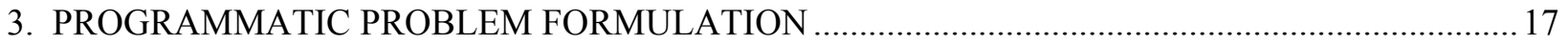

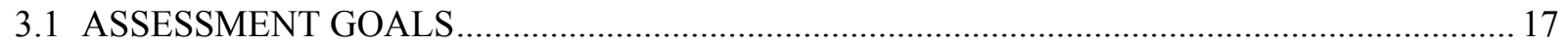

3.2 ACTIVITIES TO BE ASSESSED …………………………………………………. 20

3.2.1 Defining and Describing the Activities …………………………………………......20

3.2.2 Frequency and Intensity of the Activities......................................................................22

3.2.3 Are Activity-Specific Frameworks Available? ………………………………………......2

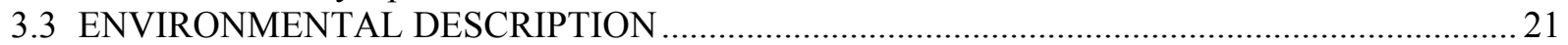

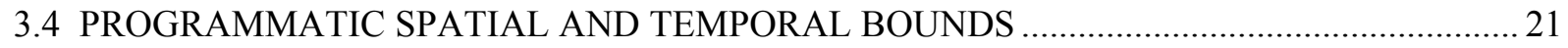

3.4.1 Combine Bounds of Activities ……………………………………………………...... 22

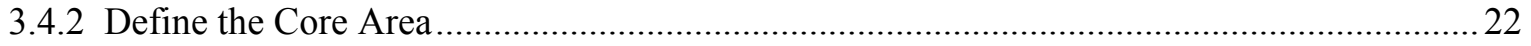

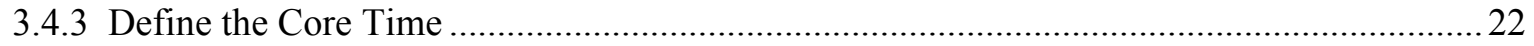

3.4.4 Define the Influence Area....................................................................................22

3.4.5 Define the Influence Time ...........................................................................................22

3.4.6 Does the Core Area Change with Time? ………………………………………….....2

3.4.7 Does the Influence Area Change With Time? ....................................................................2 24 


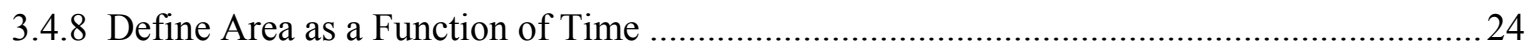

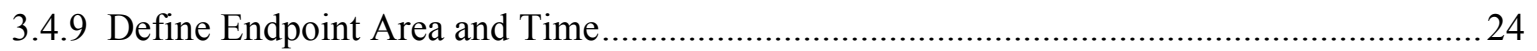

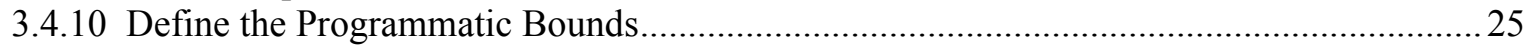

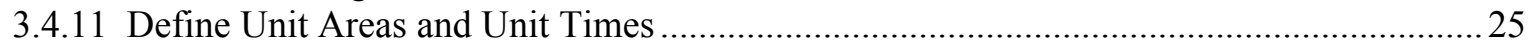

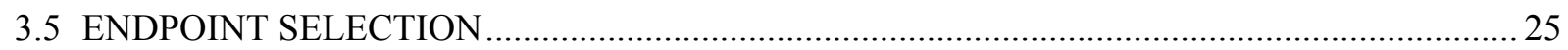

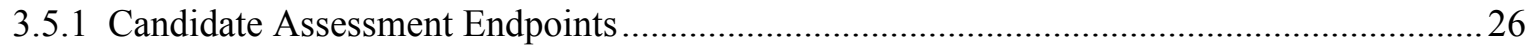

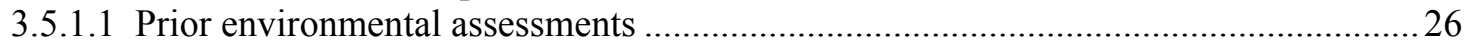

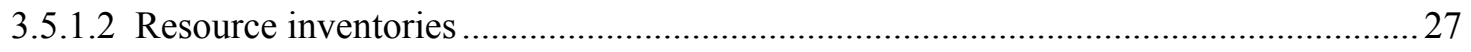

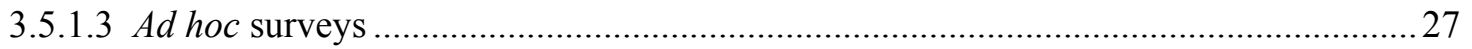

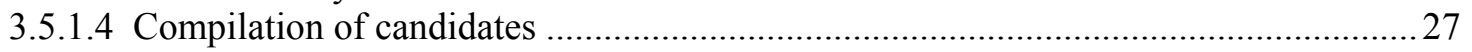

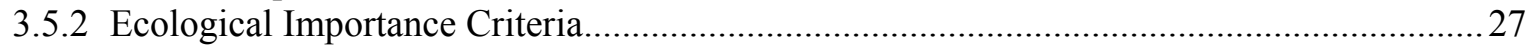

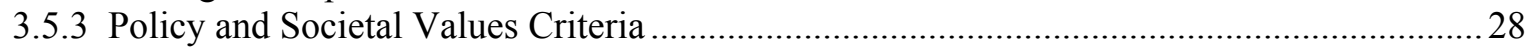

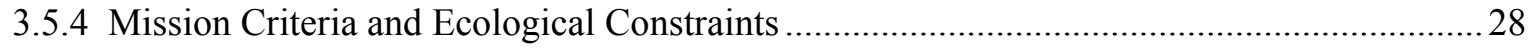

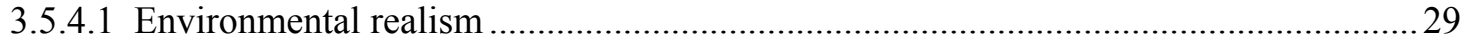

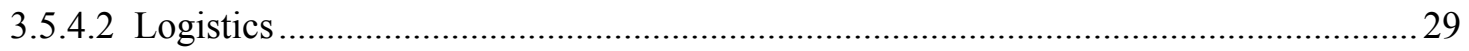

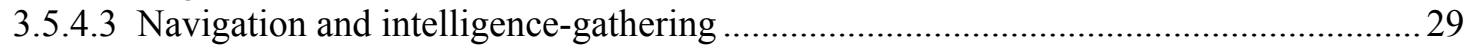

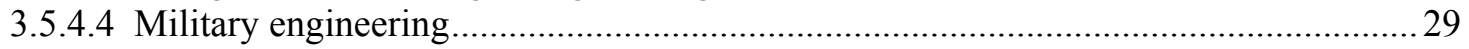

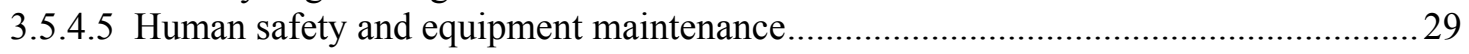

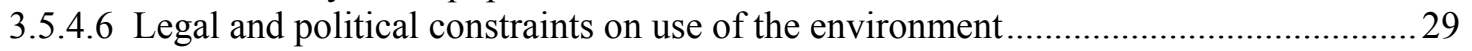

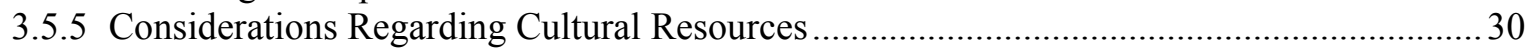

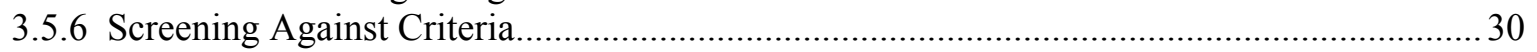

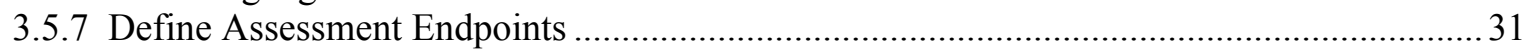

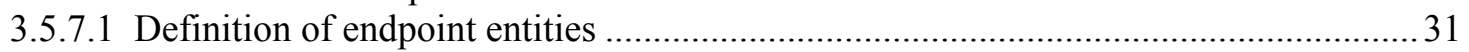

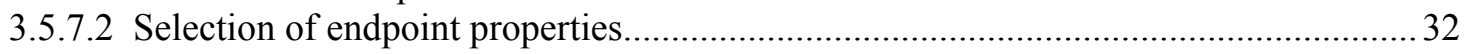

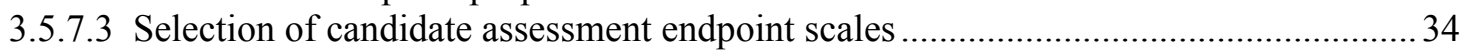

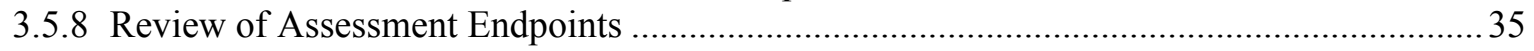

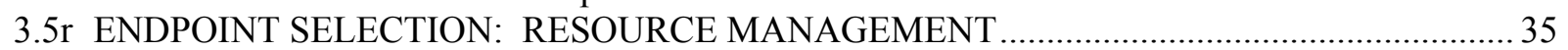

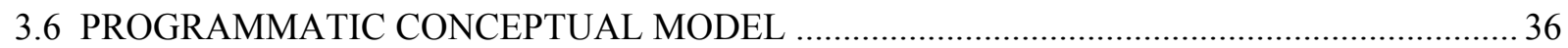

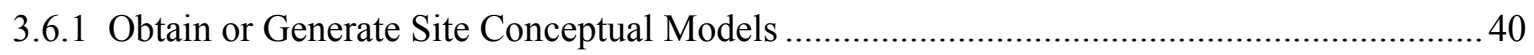

3.6.2 Obtain or Generate Receptor Conceptual Models .................................................... 42

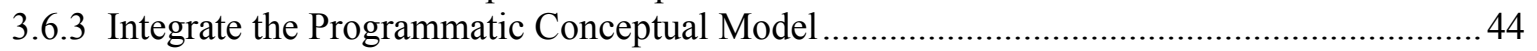

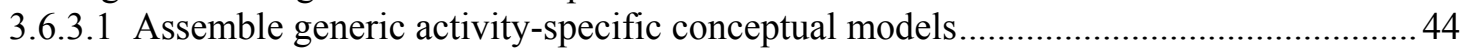

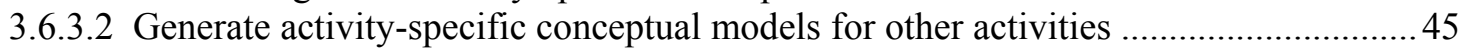

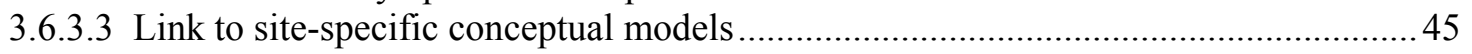

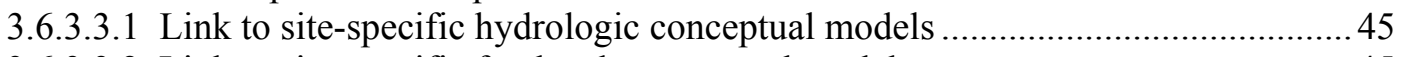

3.6.3.3.2 Link to site-specific food web conceptual models ............................................. 45

3.6.3.4 Determine spatial co-occurrence with relevant communities ................................... 45

3.6.3.5 Determine temporal co-occurrence with endpoint receptors or intermediate entities.. 45

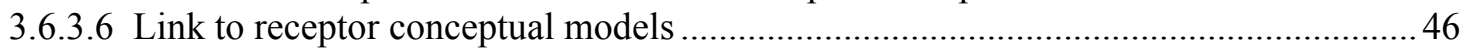

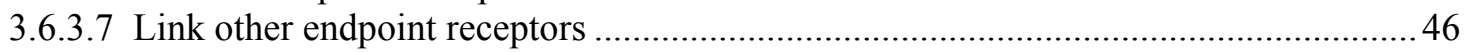

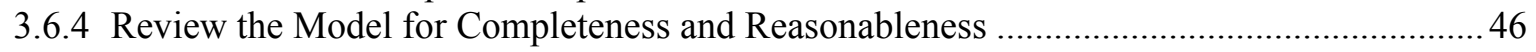

3.6r CONCEPTUAL MODELS: RESOURCE MANAGEMENT ............................................. 46

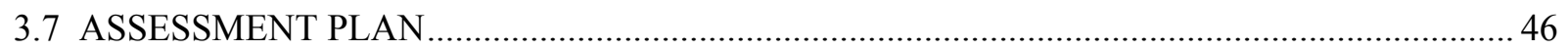

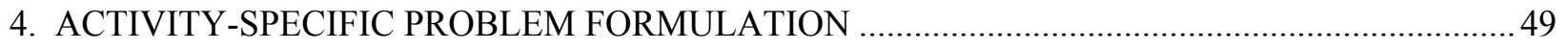

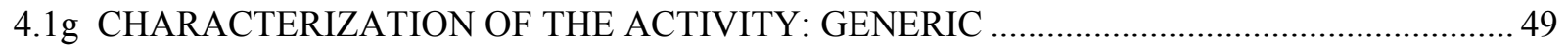

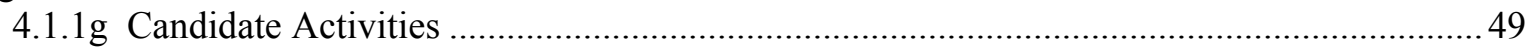

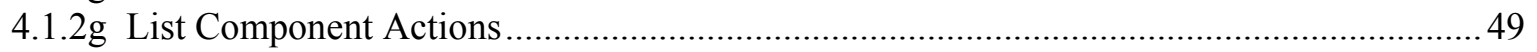

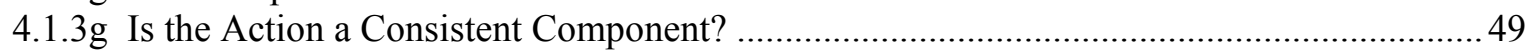

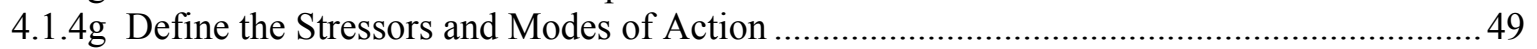

4.1i CHARACTERIZATION OF THE ACTIVITY: IMPLEMENTATION N..................................... 49

4.2g ACTIVITY-SPECIFIC ENDPOINT SELECTION: GENERIC …......................................... 50 


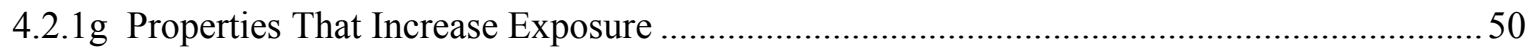

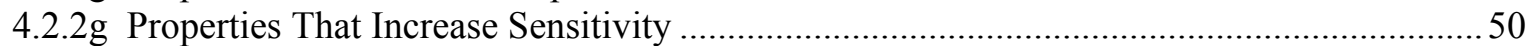

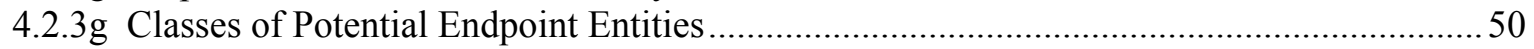

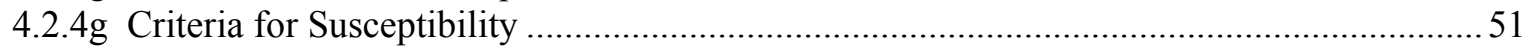

4.2.5g Generic Activity-Specific Endpoints ....................................................................... 51

4.2i ACTIVITY-SPECIFIC ENDPOINT SELECTION: IMPLEMENTATION ..............................5 52

4.3g DEFINITION OF SPATIAL AND TEMPORAL EXTENT: GENERIC .................................. 52

4.3r SPATIAL AND TEMPORAL BOUNDS: RESOURCE MANAGEMENT .............................. 52

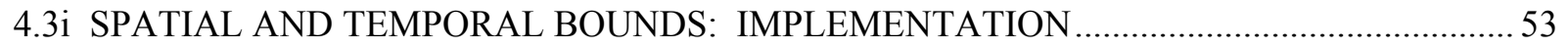

4.4g ACTIVITY-SPECIFIC CONCEPTUAL MODEL: GENERIC …........................................53

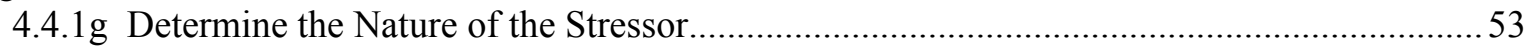

4.4.2g Define the Mode of Propagation of Physical Effects .........................................................53

4.4.3g Define the Mechanisms of Direct Physical Effects .........................................................53

4.4.4g Define the Mode of Reproduction and Spread of Introduced Organisms ...........................55

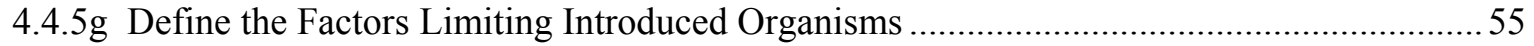

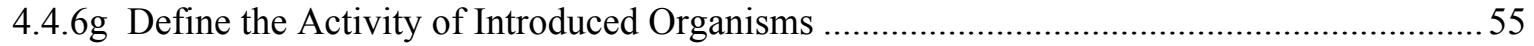

4.4.7g Describe the Transport, Deposition, and Transformation of Materials ...............................55

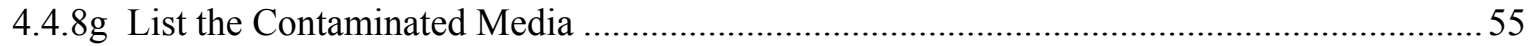

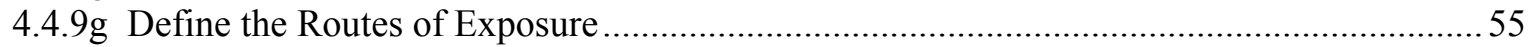

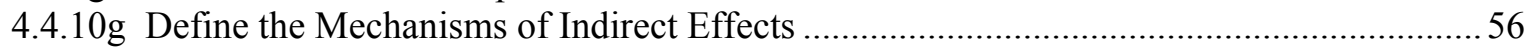

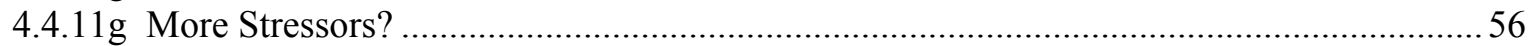

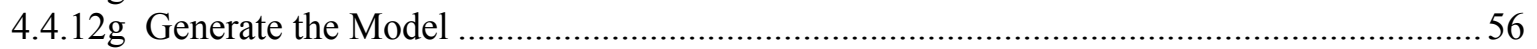

4.4i ACTIVITY-SPECIFIC CONCEPTUAL MODEL: IMPLEMENTATION ...............................56

4.5g SELECTION OF ACTIVITY-SPECIFIC MEASURES OF EXPOSURE: GENERIC ............... 56

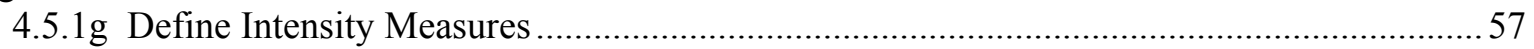

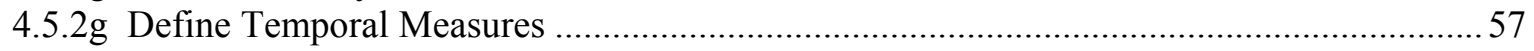

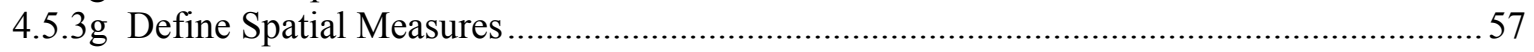

4.5i SELECTION OF ACTIVITY-SPECIFIC MEASURES OF EXPOSURE:

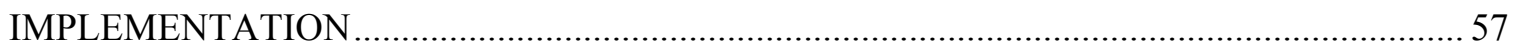

4.6g SELECTION OF ACTIVITY-SPECIFIC MEASURES OF EFFECTS.....................................58

4.6i SELECTION OF ACTIVITY-SPECIFIC MEASURES OF EFFECTS:

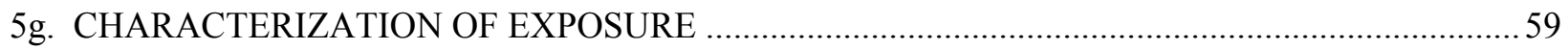

5.1g CHOOSE THE EXPOSURE ESTIMATION METHOD …...................................................... 59

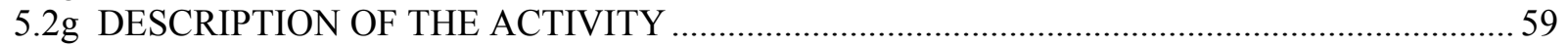

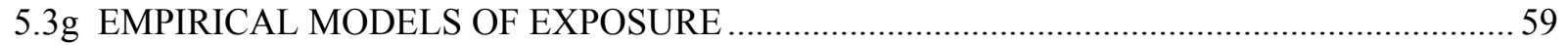

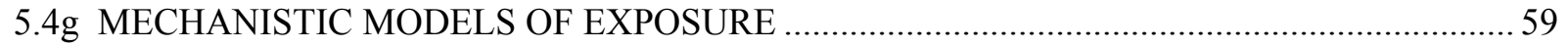

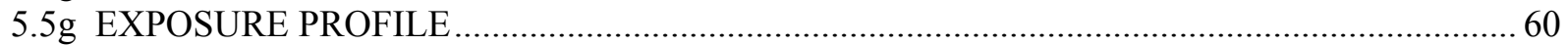

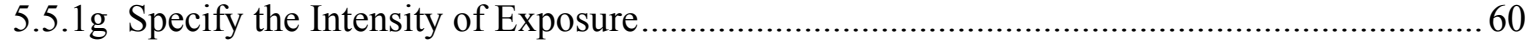

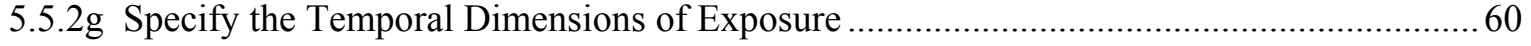

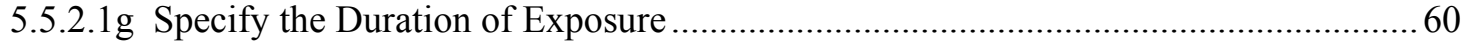

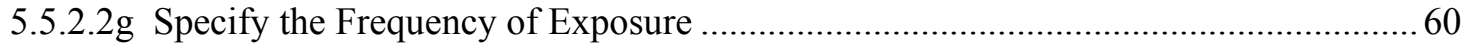

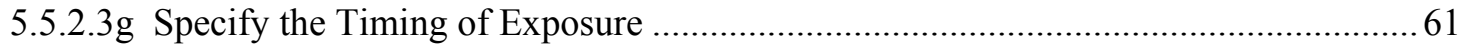

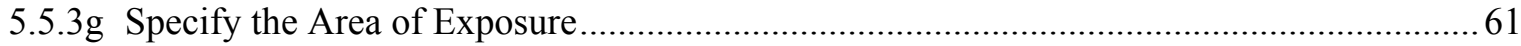

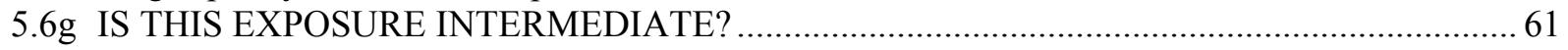

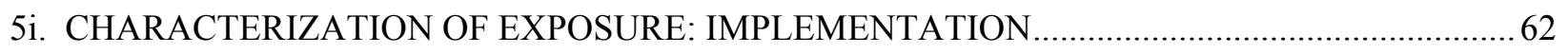

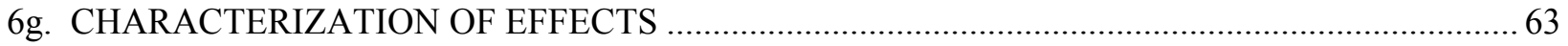

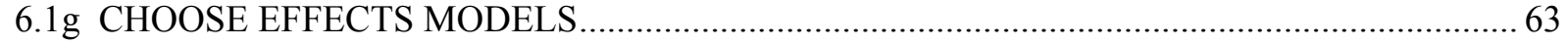

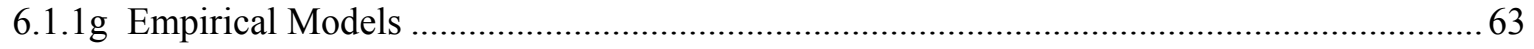

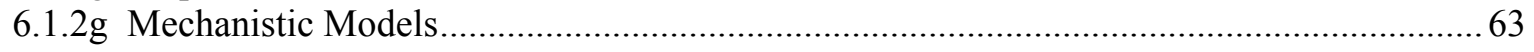


6.1.3g Threshold Models ...................................................................................................... 64

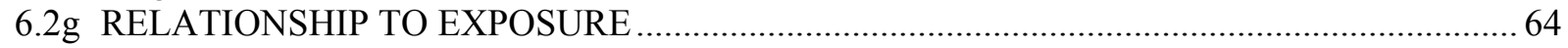

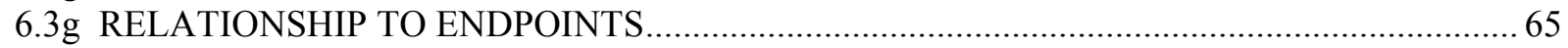

6.3.1g Extrapolation by Expert Judgment ………………………………………………….65

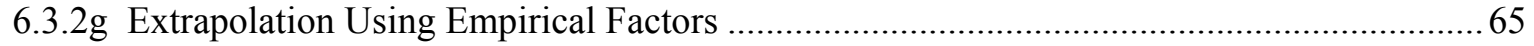

6.3.3g Extrapolation Using Empirical Models ……………………………………………....65

6.3.4g Extrapolation Using Mechanistic Models ………………………………………………...66

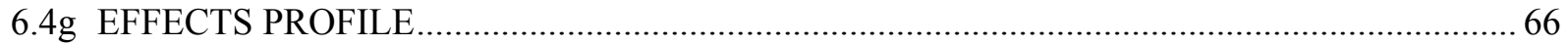

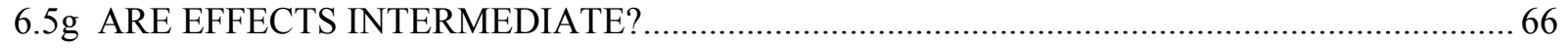

6i. CHARACTERIZATION OF EFFECTS: IMPLEMENTATION ........................................................67

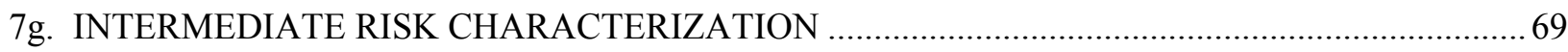

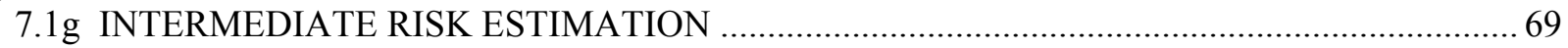

7.2g IS THE AFFECTED ENTITY AN ENDPOINT? ………………………………………......6 69

8g. ACTIVITY-SPECIFIC RISK CHARACTERIZATION ……………………………………....... 71

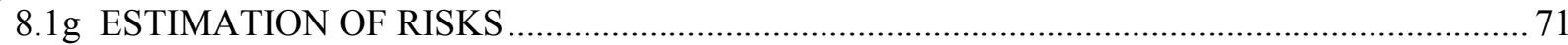

8.1.1g Implementing Exposure-Response Models ………………………………………...... 71

8.1.1.1g Empirical models ................................................................................................ 71

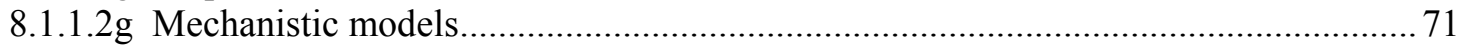

8.1.1.3g Threshold models ............................................................................................. 72

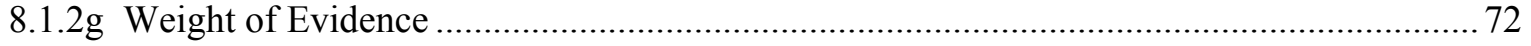

8.1.2.1g Are estimates effectively the same?.................................................................. 72

8.1.2.2g Are the lines of evidence effectively the same quality?.......................................... 72

8.1.2.3g Choose the highest quality estimate ……………………………………………..... 72

8.1.2.4g Must multiple modes of action be integrated? …………………………………..... 72

8.1.2.5g Choose the best estimate for integration ………………………………………..... 72

8.1.2.6g Equivalent estimates ......................................................................................... 73

8.1.3g Multiple Modes of Action .............................................................................................73

8.1.3.1g Are there multiple modes of action? ..................................................................... 73

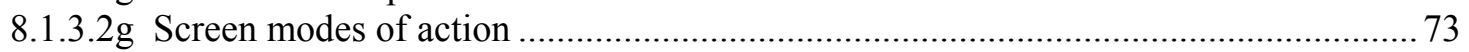

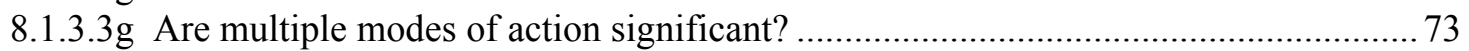

8.1.3.4g Combined Risk Models......................................................................................74

8.2g ESTIMATE UNCERTAINTY AND VARIABILITY ……………………………………..... 74

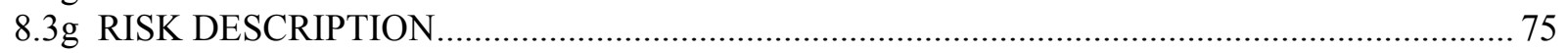

8i. ACTIVITY-SPECIFIC RISK CHARACTERIZATION: IMPLEMENTATION ………………….....76

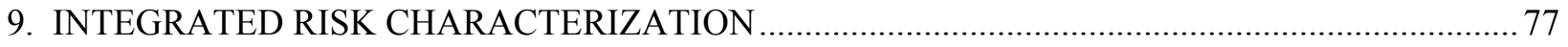

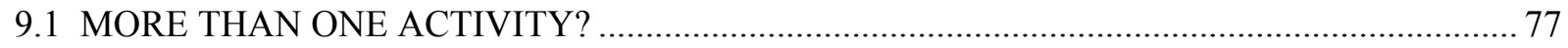

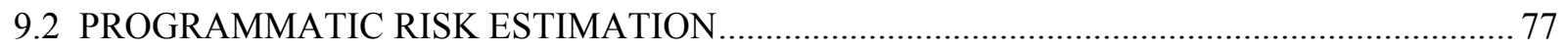

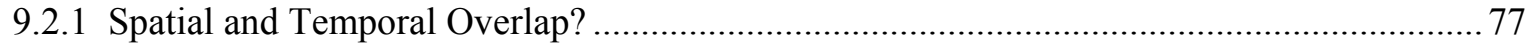

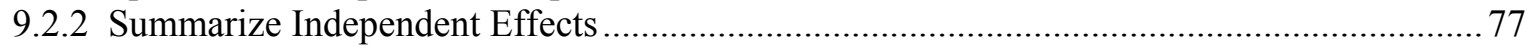

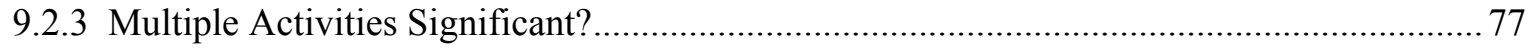

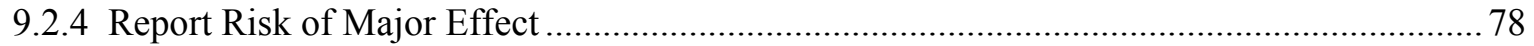

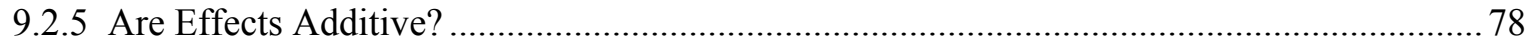

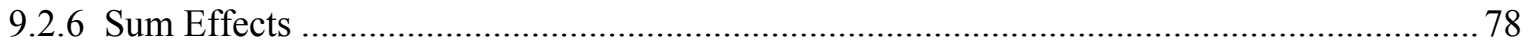

9.2.7 Are Exposures Additive?.......................................................................................... 79

9.2.8 Add Exposures and Estimate Effects..............................................................................79

9.2.9 Mechanistically Model Combined Effects ........................................................................79

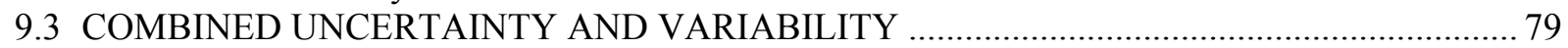

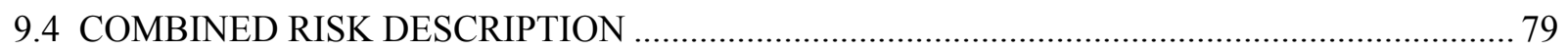




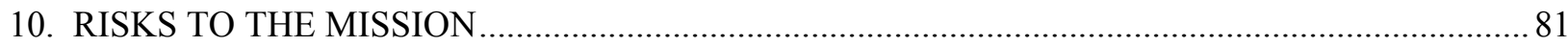

10.1 CATEGORIES OF INFORMATION NEEDED ……………............................................. 81

10.1.1 Environmental Characteristics Needed for the Military Mission ......................................... 81

10.1.1.1 Obtaining data about military environmental needs .............................................. 81

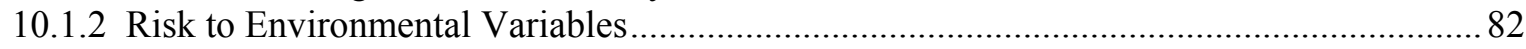

10.1.3 Risks to Mission Due to Social Valuation of Environmental Characteristics ......................82

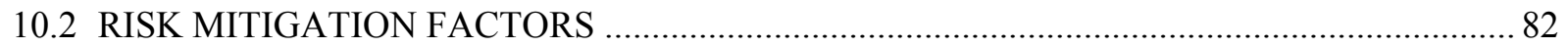

10.3 INCORPORATING ECOLOGICAL RISKS INTO MILITARY PLANNING ………………..... 82

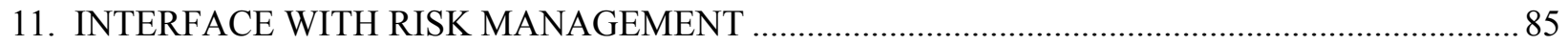

11.1 PRESENTING RESULTS TO THE DoD RISK MANAGER ……………………………....... 85

11.2 USING THE RESULTS IN DECISIONMAKING ………………………………………..... 85

11.3 USING THE RESULTS AS INPUT TO DECISION SUPPORT SYSTEMS ………………...... 85

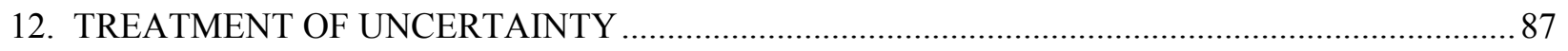

13. GLOSSARY …………………………………………………………………………… 91

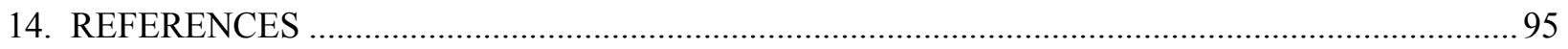

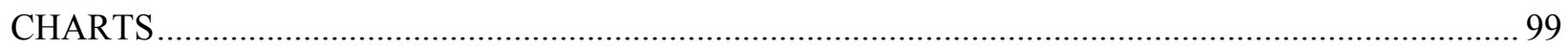

1 Military Ecological Risk Assessment Framework (MERAF)........................................... 100

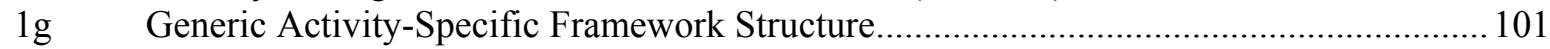

3 Programmatic Problem Formulation................................................................................ 102

3.4 Programmatic Spatial and Temporal Bounds................................................................... 103

3.5 Programmatic Assessment Endpoint Selection............................................................... 104

3.6 Programmatic Conceptual Model Development .............................................................. 105

4 Activity-Specific Problem Formulation ...…………………………………………........ 106

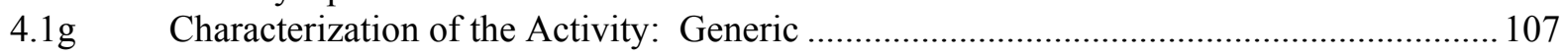

4.2g Generic Activity-Specific Endpoint Selection ................................................................. 108

4.3g Definition of Spatial and Temporal Extent ........................................................................ 109

4.3r Definition of Spatial Extent: Resource Management Activity ........................................ 110

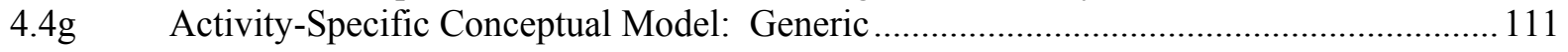

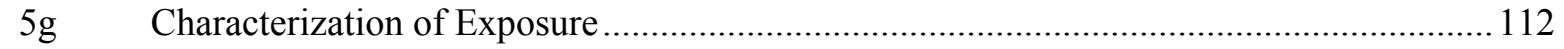

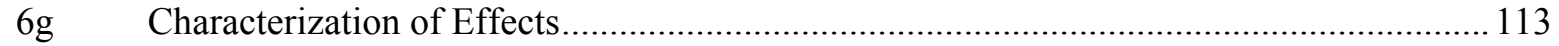

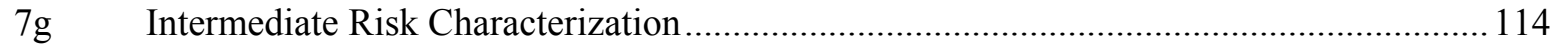

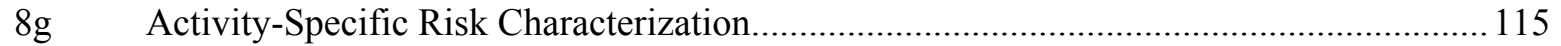

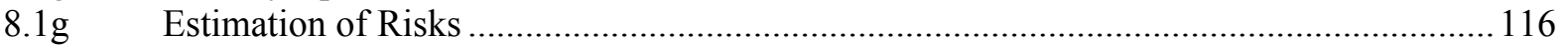

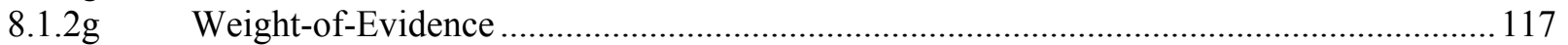

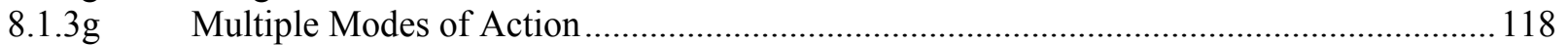

9 Integrated Risk Characterization ........................................................................... 119

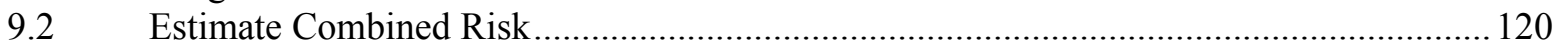




\section{LIST OF TABLES}

Page

$1 \quad$ Example ecological risk assessment goals based on given management goals .................. 18

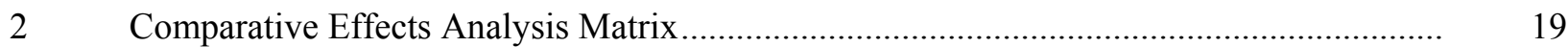

3 How to avoid common problems with assessment endpoint definitions identified

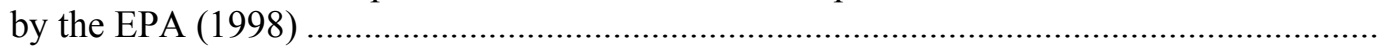




\section{LIST OF FIGURES}

Page

3.4-1 Examples of possible relationships among core, influence and endpoint areas................... 23

3.6-1 Example of a conventional conceptual model for exposure to chemicals.

The source is a chemical weapons incinerator....

3.6-2 Hypothetical activity-specific conceptual model for tank maneuvers .............................. 38

3.6-3 Hypothetical higher-level conceptual model for release of smokes ................................. 40

3.6-4 Hypothetical lower-level conceptual model for release of smokes .................................. 41

3.6-5 Assembly of a programmatic conceptual model from component conceptual models.

3.6-6 Example of a site conceptual model, the hydrology of the Oak Ridge Reservation

3.6-7 Example of a receptor conceptual model: wintering hawks in Michigan, based on research of Craighead and Craighead (1956) and modified from a diagram in Andrewartha and Birch (1984)

4.4-1 A hypothetical generic conceptual model for the activity "tank maneuvers" 


\section{ACKNOWLEDGMENTS}

This research has been funded by the U.S. Department of Defense Strategic Research and Development Program (SERDP) project CS-1054, A Risk Assessment Framework for Natural Resources on Military Training and Testing Lands.

In addition, we would like to thank Rebecca Efroymson, Dick Gebhart, and Brad Sample for comments on previous drafts of this framework. 


\section{EXECUTIVE SUMMARY}

This report provides the Department of Defense (DoD) with a framework based on a systematic, risk-based approach to assess impacts for management of natural resources in an ecosystem context. This risk assessment framework is consistent with, but extends beyond, the U.S. Environmental Protection Agency's (EPA's) ecological risk assessment framework, and specifically addresses DoD activities and management needs. Therefore, it is titled "Military Ecological Risk Assessment Framework" (MERAF) to convey this military focus.

The intended users of this report include two broad categories: 1) DoD managers and 2) technical risk assessment specialists. Depending on the scope and scale of a specific assessment and on available in-house expertise, the technical aspects of a MERAF analysis might be conducted in-house or by contracted experts. In either case, the appropriate DoD managers must be responsible for defining the scope and problem formulation, overseeing the technical analysis, and evaluating and implementing the results.

MERAF is intended to be consistent with existing procedures for environmental assessment and planning with DoD testing and training. The intention is to supplement these procedures rather than to create new procedural requirements. MERAF is suitable for use for training and testing area assessment and management. It does not include human health risks nor does it address specific permitting or compliance requirements, although it may be useful in some of these cases.

Use of MERAF fits into the National Environmental Policy Act (NEPA) process by providing a consistent and rigorous way of organizing and conducting the technical analysis for Environmental Impact Statements (EISs) (Sigal 1993; Carpenter 1995; Canter and Sadler 1997). It neither conflicts with, nor replaces, procedural requirements within the NEPA process or document management processes already in place within DoD.

MERAF is based on the EPA's framework for ecological risk assessment as elaborated in the recent proposed guidelines for ecological risk assessment (EPA 1992; EPA 1998). The terminology used in the EPA framework provides a common basis for communication among ecological risk assessors and between risk managers and risk assessors. A glossary of ecological risk terminology is provided at the end of this report.

MERAF elaborates the EPA ecological risk assessment framework to make it more useful to the DoD training and testing community and to others performing similar types of assessments. The modifications to the EPA ecological risk assessment framework that have been made to develop MERAF are explained below.

First, the modifications enable MERAF to explicitly address risks from the imposition of multiple and diverse stressors on a site. This modification is necessitated by the fact that the framework must address risks from all of the diverse activities involved in a training or testing program, and to conform with DoD policies and regulations concerning ecosystem management and the protection of natural resources (see Impact Assessment for Management of Department of Defense Natural and Cultural Resources: A Survey of Current Methods, Data, Policies and Practices with Recommendations for Future Development, Reinbold et al. 1997). At a minimum, ecosystem management requires that, rather than managing risks from individual actions, one must manage the risks to ecosystems from all of the actions that potentially affect them.

Second, the modifications explicitly address risks resulting from causal chains. That is, ecological risks may result not from the direct action of a stressor on an endpoint receptor, but from indirect effects mediated by trophic relationships, habitat requirements, etc. Although the EPA framework does not preclude the consideration of multiple stressors and indirect effects, it does not 
explicitly incorporate these issues. Therefore, it does not provide a basis for the detailed guidance required by the training and testing community.

Third, the modifications incorporate the fact that risks to natural resources imply other consequent risks. In particular, effects on the environment limit the ability to conduct training and testing activities on a site and alter the context and quality of historical and other cultural resources on a site.

MERAF is designed to address multiple and diverse actions at a particular site that potentially have combined effects on endpoint receptors. First, it provides a separate problem formulation stage for the programmatic assessment, i.e., for the training or testing program as a whole. This provides an opportunity for DoD managers to define the decision to be made, to define the activities to be performed on the site that are relevant to the decision, to characterize the site, to select endpoints, and to develop an overall conceptual model of the relationship between the training and testing activities and the receiving environment. The latter activities may, at the discretion of DoD managers, be performed in whole or in part by their contracted risk assessment specialists.

For each relevant activity, a separate subframework should be developed consisting of an activity-specific problem formulation, analysis of exposure and effects, and risk characterization. The activities addressed by these subframeworks might include operation of tracked vehicles, use of explosive munitions, or public deer hunts. The number and nature of the individual activities assessed will vary from facility to facility, among areas on a facility, and over time. Next, the results of the risk assessments for individual activities will be combined to characterize risks to the endpoints from the combined effects of all activities in the program. Finally, the results of the integrated risk characterization will be evaluated and acted upon by the relevant DoD personnel.

The definitions of the terms program and activity are flexible. The distinction is based on the fact that few decisions involve one discrete activity performed in isolation. Rather, decisions involve multiple activities or a single activity added to a set of activities that are already being performed at a site. The term program is used here to designate $a$ set of activities that are carried out to accomplish a mission and about which a decision must be made. Examples include a training exercise for helicopter reconnaissance or a series of tests for a set of new obscurants. Each activity is a distinct set of actions that make up the program. Examples include driving tanks, firing live ammunition, releasing an obscurant, or monitoring a test. Programs and activities that do not actually involve training or testing may also occur on a facility. An example is a resource management program in which the individual activities might include controlled burns, timber harvests, or public hunts.

A program may be carried out at more than one range, facility, or location. If the number of such sites is small, one can assess the risks of the program by simply assessing the risks at all sites. One example might be the Navy's ship-shock testing, where the risks of environmental impacts must be considered in the same systematic way, programmatically, regardless of where each test may occur. However, in some cases, the number of sites may be large or the set of sites may not have been defined. In such cases, the programmatic and the activity levels of MERAF can be implemented, but the sitespecific elements of MERAF cannot. In their place, default assumptions or ranges of conditions may be used in place of site-specific information.

Military training and testing programs are typically characterized by multiple activities generating multiple agents and by multiple occurrences of those activities. The exposures, effects, and risks associated with multiple activities or agents are referred to as "combined." Those that are associated with multiple occurrences of an activity or agent are referred to as "cumulative." Cumulative risks may or may not require special attention depending on conditions and the goals of the assessment. If the goal is simply to characterize the risks associated with a particular proposed training or testing program, one may take the current state of the site as a baseline and estimate the state following the program. This requires no change in MERAF and is analogous to the estimation of risks to a resource population from increased harvesting rates given a prior rate. MERAF provides instruction on how to perform such cumulative 
assessments. In other cases, the goal may be to assess the risks from a pattern of cumulative exposures and effects because of concerns about the long-term sustainability of both the natural resources and the military mission. In those cases, it is necessary to explicitly model the temporal pattern of exposure, effects, recovery, re-exposure, etc. This particular cumulative risk assessment would be performed by the same process as that presented in MERAF, but the environmental responses to the iteration of an activity would need to be simulated. Assessments of that complexity would need to be planned ad hoc because no reasonable framework could represent an assessment process that would be applicable to the many combinations of activities and sites.

MERAF is intended to apply to risk assessments for resource management plans as well as to training and testing programs. There are potentially significant advantages to including resource management in the same framework as training and testing activities. First, developing a common set of endpoints ensures that the activities do not have conflicting goals and that the risks are judged in a consistent manner. For example, while a training commander and the natural resources managers may agree on the desirability of a "healthy environment," there could be conflicts if the natural resource managers plan to develop an artificial wetland that would block an important training maneuver corridor. In a case such as this, the common endpoint could be the restoration and maintenance of a more trainingresilient dry-land ecosystem along the corridor. Second, the risks to a resource from training and testing activities depend on the resource management activities. That is, activities that might pose an unacceptable risk when considered in isolation might be acceptable given appropriate resource management activities (e.g., if habitat restoration exceeds habitat destruction). On the other hand, training and testing activities may negate the potential benefits of a given resource management plan.

Three types of risks are associated with resource management plans. First, there is a risk that the plan will not achieve the management goal for the resource and may even have unintended negative effects. Second, resource management activities have effects on species and communities other than the resource being managed and, inevitably, some of these are negative. For example, setting fires to enhance populations of a fire-dependent plant will have negative effects on plants and animals that are not firetolerant or that require the habitat that occurs in the absence of fire. Third, resource management activities may modify the environment in ways that place the military mission at risk.

In addition to risks to the environment from training and testing activities, risks to the military mission due to changes in the state of the environment must be considered. Destruction of vegetation, churning of wetland soils, rutting of slopes, etc. can create safety hazards, make a training range unrealistic as a simulated battlefield, and may inhibit or even prevent passage of troops and equipment. Environmental effects of training and testing, real or perceived, can also pose risks to efficient accomplishment of the military mission due to political, legal, and other pressures brought to bear by regulators, interest groups, Native American tribes, and the general public. The risks considered here are risks to future training and testing missions from effects of current training and testing activities. Hence, the logic portrayed in Chart 1 is that training and testing activities have effects on the environment, the integration of those effects results in an estimated state of the environment, which imposes a risk on future missions that may include the same or different activities. MERAF does not include the actual methods for estimating risk to the mission, which would include considerations other than risks to the environment, such as risks to the troops from contamination of the range or availability of alternate facilities or ranges for performing the mission. However, MERAF does include methods for linking the ecological risk assessment to the assessment of risks to the mission (Sec. 10).

MERAF is more prescriptive than the EPA ecological risk assessment framework. That is, it will describe more specifically what should be done and how it should be done at each stage in the process. This is possible because, unlike the EPA framework, MERAF addresses risks from a limited set of activities in a relatively limited set of circumstances.

An algorithmic approach, such as may be used for single-source industrial contaminants, is not feasible for ecological risk assessments. Ecological risk assessments are more complex and less 
standardized because of the large number of potential endpoints, the highly variable spatial and temporal scales, the large number of poorly defined interactions among components of the systems, and the multiple lines of evidence that must often be integrated.

Rather than an algorithm, we believe that the appropriate paradigm for MERAF is the concept of schema, adapted from psychology by M. Gell-Mann (1994). A schema is a set of rules developed by a complex adaptive system for responding to a class of situations that are based on apparent regularities in the class. Examples of schemata are the genetic instructions that determine how an organism responds to the environment and the set of rules that a child develops to decode and generate language. Schemata, unlike algorithms, are incomplete and provisional, and they evolve. The evolution of MERAF through the addition and replacement of modular components is consistent with the concept of a schema. The modular components of MERAF can be replaced like individual genes are replaced without scrapping the entire genome, or modules can be added to refine MERAF like newly learned irregular verb forms are added along with regular verb declensions. In this case, the complex adaptive system developing the schema is the community of assessment scientists and users and managers of the environment working on DoD training and testing facilities.

The MERAF approach has a number of potential advantages. First, its modularity can increase efficiency in risk assessment. If one attempts to integrate the entire risk assessment process, then any change in the proposed activities requires a complete re-analysis of the risks. Using MERAF, however, if any activity is added, deleted, or modified, the assessments of the other activities are retained and only the changed activity and the integrated risk characterization must be modified. In addition, the MERAF structure allows the independent development of risk assessment methods for individual activities so that the capability to assess risks of multiple activities can be developed step-wise. That is, rather than develop a method for integrated assessments of risks of tracked vehicles, smokes, and explosions, one could develop a method for tracked vehicles, a method for smokes (with interactions with the method for tracked vehicles), and a method for explosions (with interactions with the methods for tracked vehicles and smokes).

Similar to the EPA framework for ecological risk assessment, MERAF does not incorporate tiered assessment explicitly. Because of the diversity of assessments to which MERAF must be applied, no one approach to defining tiers is likely to be useful. In addition, the authors believe that tiering is unlikely to be routinely employed in assessments of training or testing activities.

MERAF can be used in tiered assessments when appropriate. Following the risk characterization, it may be decided that the risks are not adequately characterized and additional data collection or analysis is needed. In such cases, the results of the risk characterization are input to the next tier of problem formulation. As activity-specific frameworks are developed, activities for which tiered assessment is appropriate will be noted and incorporated into the frameworks. Those activities would be those for which the assessor would benefit from a narrowing of the issues to be assessed, and for which there are quick and conservative methods appropriate for screening, as well as more realistic methods appropriate for definitive assessments.

This report is organized based on linked charts and text. The charts, which are presented at the end of the report, portray the conceptual relationships among tasks, the order in which they are performed, and the flow of information from one task to another. A common numbering system is used to link the charts and text. The overall conceptual framework of MERAF is illustrated in Chart 1 . The major section numbers of this report correspond to box numbers in Chart 1 , and subsections correspond to boxes in charts that elaborate the components of Chart 1. 


\section{INTRODUCTION}

\subsection{BACKGROUND AND OBJECTIVE}

\subsubsection{Background}

The Department of Defense (DoD) needs a systematic, consistent, risk-based approach to assess impacts for management of natural resources in an ecosystem context. Based on review of current methods, data, policies, and practices for impact assessment used by or relevant to DoD, recommendations were made for development of an ecological risk assessment framework for DoD (Reinbold et al. 1997). It is policy to implement ecosystem management on DoD installations (Deputy Undersecretary of Defense (Environmental Security) [DUSD(ES)] 1994). The 1995 Defense Science Board on Environmental Security recommended an integrated and proactive conservation program within a risk management framework. To meet these objectives, a more specific and detailed approach is required for use by DoD personnel responsible for implementing them.

The intended users of this report include two broad categories: 1) DoD managers and 2) technical risk assessment specialists. Depending on the scope and scale of a specific assessment and on available in-house expertise, the technical aspects of a Military Ecological Risk Assessment Framework (MERAF) analysis might be conducted in-house or by contracted experts. In either case, the appropriate DoD managers must be responsible for defining the scope and problem formulation, overseeing the technical analysis, and evaluating and implementing the results.

\subsubsection{Objective}

The objective of this research is to provide the DoD with a framework based on a systematic, risk-based approach to assess impacts for management of natural resources in an ecosystem context. This risk assessment framework is consistent with, but extends beyond, the EPA's ecological risk assessment framework, and specifically addresses DoD activities and management needs.

MERAF is intended to be consistent with existing procedures for environmental assessment and planning with DoD testing and training. The intention is to supplement these procedures rather than creating new procedural requirements. MERAF is suitable for use for training and testing area assessment and management. It does not include human health risks nor does it address specific permitting or compliance requirements, although it may be useful in some of these cases.

Use of MERAF fits into the National Environmental Policy Act (NEPA) process by providing a consistent and rigorous way of organizing and conducting the technical analysis for Environmental Impact Statements (EISs) (Sigal 1993; Carpenter 1995; Canter and Sadler 1997). It neither conflicts with, nor replaces, procedural requirements within the NEPA process or document management processes already in place within DoD.

\subsection{APPROACH AND STRATEGY}

The framework is based on the EPA's framework for ecological risk assessment as elaborated in the recent proposed guidelines for ecological risk assessment (EPA 1992; EPA 1998). This was done for consistency as well as utility. The EPA framework has become the standard basis for ecological risk assessment in the federal government, including the tri-services guidance for contaminated sites (Wentsel et al. 1996). Its structure is consistent with prior human health and ecological risk assessment paradigms as well as the National Research Council's ecological risk assessment paradigm (NRC 1983; Barnthouse and Suter 1986; NRC 1993). More importantly its structure is an appropriate basis for performing defensible and useful risk assessments for a variety of ecosystems and stressors. Finally, the terminology used in the EPA framework provides a common basis for communication among ecological risk assessors 
and between risk managers and risk assessors. A glossary of ecological risk terminology is provided at the end of this report.

This framework elaborates the EPA ecological risk assessment framework to make it more useful to the DoD training and testing community and to others performing similar types of assessments.

First, it has been modified to explicitly address risks from the imposition of multiple and diverse stressors on a site. This modification is necessitated by the fact that the framework must address risks from all of the diverse activities involved in a training or testing program and to conform with DoD policies and regulations concerning ecosystem management and the protection of natural resources (see Reinbold et al. 1997). Ecosystem management, at a minimum, requires that, rather than managing risks from individual actions, one must manage the risks to ecosystems from all of the actions that potentially affect them. In contrast, the EPA framework and its predecessors were developed for assessing risks of an individual action involving the release of a contaminant such as registering a new pesticide or issuing a permit for an effluent. The EPA is beginning to develop methods for multi-stressor risk assessment, but they are not sufficiently developed for the purpose of this project (Science Policy Council 1997).

Second, it has been modified to explicitly address risks resulting from causal chains. That is, ecological risks may result not from the direct action of a stressor on an endpoint receptor, but from indirect effects mediated by trophic relationships, habitat requirements, etc. The EPA framework does not preclude the consideration of multiple stressors and indirect effects, and the EPA and others have used it in such situations. Because the EPA framework does not explicitly incorporate these issues, however, it does not provide a basis for the detailed guidance required by the training and testing community.

Third, it has been augmented to incorporate the fact that risks to natural resources imply other consequent risks. In particular, effects on the environment limit the ability to conduct training and testing activities on a site and modify the context and quality of historical and other cultural resources on a site.

\subsubsection{Adaptation for Place-Based and Programmatic Assessments}

This conceptual framework of MERAF is designed to address multiple and diverse actions at a particular place that potentially have combined effects on endpoint receptors (Chart 1; charts appear at the end of the report). First, it provides a separate problem formulation stage for the programmatic assessment, i.e., for the training or testing program as a whole. This provides an opportunity for the DoD manager, with support from contracted specialists if needed, to define the decision to be made, define the activities to be performed on the site that are relevant to the decision, characterize the site, select endpoints, and develop an overall conceptual model of the relationship between the training and testing activities and the receiving environment. For each relevant activity, a separate subframework should be developed consisting of an activity-specific problem formulation, analysis of exposure and effects, and risk characterization. The activities addressed by these subframeworks might include operation of tracked vehicles, use of explosive munitions, or public deer hunts. The number and nature of the individual activities assessed will vary from facility to facility, among areas on a facility, and over time. Next, the results of the risk assessments for individual activities will be combined to characterize risks to the endpoints from the combined effects of all activities in the program. In cases where specialists have been contracted to perform a substantial amount of these tasks, the results of the integrated risk characterization will be conveyed to the DoD manager who may make an implementation decision or ask for another iteration of risk assessment to resolve uncertainties.

The definitions of the terms program and activity are flexible. The distinction is based on the fact that few decisions involve one discrete activity performed in isolation. Rather, decisions involve multiple activities or a single activity added to a set of activities that are already being performed at a site. The term program is used here to designate $a$ set of activities that are carried out to accomplish a mission and about which a decision must be made. Examples include a training exercise for helicopter reconnaissance or a series of tests for a set of new obscurants. Each activity is a distinct set of actions that make up the 
program. Examples include driving tanks, firing live ammunition, releasing an obscurant, or monitoring a test. Programs and activities that do not actually involve training or testing may also occur on a facility. An example is a resource management program in which the individual activities might include controlled burns, timber harvests, or public hunts.

A program may be carried out at more than one range, facility, or location. If the number of such sites is small, one can assess the risks of the program by simply assessing the risks at all sites. One example might be the Navy's ship-shock testing, where the risk of environmental impacts must be considered in the same systematic way, programmatically, regardless of where each test may happen to occur. In some cases, however, the number of sites may be large or the set of sites may not have been defined. In such cases, the programmatic and the activity levels of the framework can be implemented, but the site-specific elements of the framework cannot. In their place, default assumptions or ranges of conditions may be used in place of site-specific information.

This approach has a number of potential advantages. First, its modularity can increase efficiency in risk assessment. If one attempts to integrate the entire risk assessment process, then any changes in the proposed activities require a complete reanalysis of the risks. Using MERAF, however, if an activity is added, deleted, or modified, the assessments of the other activities are retained and only the changed activity and the integrated risk characterization must be modified. In addition, this structure allows the independent development of risk assessment methods for individual activities so that the capability to assess risks of multiple activities can be developed step-wise. That is, rather than develop a method for integrated assessments of risks of tracked vehicles, smokes, and explosions, one could develop a method for tracked vehicles, a method for smokes (with interactions with the method for tracked vehicles), and a method for explosions (with interactions with the methods for tracked vehicles and smokes).

This approach depends on an assumption that processes that control the exposure and response to the agents have some degree of independence so that the integrated assessment can be performed by determining the consequences of combined effects that are estimated separately. For example, we can estimate the dose of smoke received by an animal and the toxic effect of that dose without knowing whether tracked vehicles have damaged the habitat. Assessment of the tracked vehicle effects on the habitat can be performed independently of the effects of smoke used during the exercise. The integrated risk characterization can be based on the interaction of effects such as reduced abundance due to effects of smokes and due to habitat destruction by tracked vehicles (taking into consideration spatial and temporal relationships between the effects). However, it is possible that there are fundamental interactions. For example, habitat modification by tracked vehicles may change the behavior of an animal rather than simply changing its abundance. The risk assessments for the two activities would have to be completely integrated if that interaction occurred, if it were important to the results of the assessment, and if there were some scientific basis for estimating the interaction. If such interactions were common and important, this approach would not be feasible. Based on experience, however, we believe that, in many cases, they are not. Determining the appropriateness of this assumption will be part of the research performed by this project.

\subsubsection{Indirect Effects}

Conventional ecological risk assessments develop an exposure profile and an exposure-response relationship, which are brought together in the risk characterization. This approach is appropriate for direct effects such as toxic effects of exposure to a chemical or effects of concussion from an explosion. Risks to endpoint entities, however, are due to indirect effects for many, if not most, training and testing activities. In particular, many risks are due to loss or modification of habitat. Chart $1 g$ shows how the analysis phase of generic ( $\mathrm{g}$ ) activity-specific frameworks has been modified to accommodate assessment of risks due to indirect effects.

The induction of risks through indirect exposures can best be thought of as resulting from a series of cause-effect linkages (i.e., a causal chain). In a risk assessment framework, each cause-effect linkage 
consists of exposure to a stressor (analysis of exposure) and effects of that exposure (risk characterization), which are determined by the relevant exposure-response relationship (analysis of effects). The effect, estimated in a risk characterization step, constitutes a new stressor to which a new set of receptors is exposed. Hence, a causal chain can be thought of as an exposure-response loop, as represented in the analysis phase of Chart $1 g$. The loop terminates when the affected entity is the chosen assessment endpoint.

For example, tracked vehicle traffic (stressor), imposed on a meadow with a certain intensity and frequency (analysis of exposure 1), causes changes in the plant community of the meadow (intermediate risk characterization 1), which is determined by the relationship between exposure to tracked vehicles and growth and survival of plants (analysis of effects 1). That change in the meadow plant community is a stressor to which a wildlife population may be exposed (analysis of exposure 2), which causes changes in the abundance of the population (intermediate risk characterization 2), based on the relationship between habitat quality and population abundance (analysis of effects 2). One could hypothesize a third loop if a predator of the wildlife population were also an endpoint species. When all of the endpoint entities on that particular causal chain are included, the results of the analysis are passed to the risk characterization for the activity.

The representation of indirectly induced ecological risks as causal chains was proposed in the issue paper on exposure assessment prepared for the EPA guidelines development (Suter et al. 1994). MERAF takes the additional steps of generalizing chains of varying length as loops and incorporating them into a risk framework.

\subsubsection{Risks to the Mission}

In addition to risks to the environment from training and testing activities, risks to the military mission due to changes in the state of the environment must be considered. Destruction of vegetation, churning of wetland soils, rutting of slopes, etc. can make a training range unrealistic as a simulated battlefield and may inhibit or even prevent passage of troops and equipment. Environmental effects of training and testing, real or perceived, can also pose risks to efficient accomplishment of the military mission due to political, legal, and other pressures brought to bear by regulators, interest groups, Native American tribes, and the general public. The risks considered here are risks to future training and testing missions from effects of current training and testing activities. Hence, the logic portrayed in Chart 1 is that training and testing activities have effects on the environment, the integration of those effects results in an estimated state of the environment, which imposes a risk on future missions that may include the same or different activities. MERAF does not include the actual methods for estimating risk to the mission, which would include considerations other than risks to the environment such as risks to the troops from contamination of the range or availability of alternate facilities or ranges for performing the mission. However, MERAF does include methods for linking the ecological risk assessment to the assessment of risks to the mission (Sec. 10).

\subsubsection{Comparative Assessment}

NEPA is a major impetus for the assessments performed at DoD training and testing facilities (Reinbold et al. 1997). NEPA assessments emphasize the comparison of alternatives (CEQ 1986). In contrast, human health and ecological risk assessment frameworks have emphasized the determination of the nature, magnitude, and likelihood of effects so as to determine their acceptability. A fundamental problem for comparative assessments is the development of a common scale on which to base the comparison.

A direct, risk-based approach to comparative assessment is to perform a risk assessment on each alternative. That is, one would perform steps two through eight in Chart 1 for each alternative form of the training or testing program including the NEPA-mandated "no action" alternative. This could be done 
efficiently because many of the components of each assessment would be common to most alternatives. Some components, such as the selection of endpoints and the definition of the scope of the assessment, must be common to all alternatives in order to perform the comparison. For example, if an endpoint were the probability of the local extinction of an endangered species on a gunnery range, then all alternatives could be compared based on that common metric. A limited set of clearly defined endpoints allows comparison of apples to apples and oranges to oranges. However, it does not generate a single metric that integrates across endpoints for comparison of alternatives. It is left to the risk management process to decide whether an alternative that creates a risk of local extinction of an endangered species is preferable to one that destroys a wetland. That is a matter of values, not science.

An alternative approach to comparative assessment that has been recommended for DoD facilities is the use of scoring systems (Reinbold et al. 1997). Scoring systems rank alternatives based on qualitative or semi-quantitative scores. That is, for each alternative, the components of the assessment are assigned scores such as low, moderate, and high; those component scores are assigned numeric values such as low $=1$, moderate $=2$, and high $=3$; and the numeric scores are added or otherwise combined to yield a total score. The advantages of scoring systems are that they can be implemented when there is little basis for assessment beyond expert judgment, they can be quickly and relatively easily implemented, and, because they are basically qualitative, they can be used to compare dissimilar effects. The disadvantage of qualitative scoring systems is that although they rank alternatives, they do not indicate the expected nature or magnitude of effects. Therefore, all of the alternatives may be acceptable, none may be acceptable, or there may be a mixture of acceptable and unacceptable alternatives. In addition, the use of such scoring systems to compare alternative actions can subsume value judgments in the technical process of assessment.

This framework will, as far as possible, be risk-based and quantitative. However, because it depends on the current state-of-knowledge and practice, it will need to incorporate qualitative components that are currently used by the DoD.

\subsubsection{Cumulative Versus Combined Risks}

Traditionally, risk assessments have focused on an individual occurrence of a hazardous agent. Military training and testing programs are typically characterized by multiple activities generating multiple agents and by multiple occurrences of those activities. The exposures, effects, and risks associated with multiple activities or agents are referred to as "combined." Those that are associated with multiple occurrences of an activity or agent are referred to as "cumulative." When combined risks occur, they must be estimated, and this report devotes considerable attention to their estimation. Cumulative risks may or may not require special attention depending on conditions and the goals of the assessment. If the goal is to simply characterize the risks associated with a particular proposed training or testing program, one may take the current state of the site as a baseline and estimate the state following the program. This requires no change in MERAF and is analogous to the estimation of risks to a resource population from increased harvesting rates given a prior rate. MERAF provides instruction on how to perform such cumulative assessments. In other cases, the goal may be to assess the risks from a pattern of cumulative exposures and effects because of concerns about the long-term sustainability of both the natural resources and the military mission. In those cases, it is necessary to explicitly model the temporal pattern of exposure, effects, recovery, re-exposure, etc. This particular cumulative risk assessment would be performed by the same process as that presented in MERAF, but the environmental responses to the iteration of an activity would need to be simulated. Assessments of that complexity would need to be planned ad hoc because no reasonable framework could represent an assessment process that would be applicable to the many combinations of activities and sites. 


\subsubsection{Provision of Guidance}

MERAF is more prescriptive than the EPA ecological risk assessment framework. That is, it will describe more specifically what should be done and how it should be done at each stage in the process. This is possible because, unlike the EPA framework, MERAF addresses risks from a limited set of activities in a relatively limited set of circumstances.

Ideally, the framework would be algorithmic. That is, it would prescribe exactly what should be done and how, in order to complete an ecological risk assessment for a training or testing program. Some risk assessments are sufficiently standardized to be reduced to algorithms, including human health risk assessments of contaminated sites under CERCLA, engineering risk assessments for aircraft and nuclear power plants, and financial risk assessments for insurance companies and investors. However, that is not feasible for ecological risk assessments. Ecological risk assessments are more complex and less standardized because of the large number of potential endpoints, the highly variable spatial and temporal scales, the large number of poorly defined interactions among components of the systems, and the multiple lines of evidence that must often be integrated.

Rather than an algorithm, we believe that the appropriate paradigm for this framework is the concept of a schema, adapted from psychology by M. Gell-Mann (1994). A schema is a set of rules developed by a complex adaptive system for responding to a class of situations that are based on apparent regularities in the class. Examples of schemata are the genetic instructions that determine how an organism responds to the environment and the set of rules that a child develops to decode and generate language. Schemata, unlike algorithms, are incomplete and provisional, and they evolve. The evolution of MERAF through the addition and replacement of modular components is consistent with the concept of a schema. The modular components of MERAF can be replaced like individual genes are replaced without scrapping the entire genome, or modules can be added to refine MERAF like newly learned irregular verb forms are added along with regular verb declensions. In this case, the complex adaptive system developing the schema is the community of assessment scientists and users and managers of the environment working on DoD training and testing facilities. The purpose of this project is to develop a structure for the schema and, in the second phase, to develop components of the schema for specific highpriority activities drawing on the experience of the community of assessment scientists and user experience that has not previously been collected and organized.

\subsubsection{Tiered Assessment}

It is common practice to conduct ecological risk assessments iteratively. The advantage of this approach is that simple assessment approaches can be used initially to help narrow the problem so that more data and modeling-intensive approaches can be applied to a small set of stressors, receptors, or areas. This approach is particularly useful in assessments of contaminated sites for which screening assessments can identify the small fraction of chemicals that merit detailed assessment (Wentsel et al. 1996). Similar to the EPA framework for ecological risk assessment, MERAF does not incorporate tiered assessment explicitly. Because of the diversity of assessments to which MERAF must be applied, no one approach to defining tiers is likely to be useful. In addition, the authors believe that tiering is unlikely to be routinely employed in assessments of training or testing activities.

MERAF can be used in tiered assessments when appropriate. Following the risk characterization, it may be decided that the risks are not adequately characterized and additional data collection or analysis is needed. In such cases, the results of the risk characterization are input to the next tier of problem formulation. As activity-specific frameworks are developed, activities for which tiered assessment is appropriate will be noted and incorporated into the frameworks. Those activities would be those for which the assessor would benefit from a narrowing of the issues to be assessed, and for which there are quick and conservative methods appropriate for screening, as well as more realistic methods appropriate for definitive assessments. 


\subsubsection{Geographic Information Systems}

Geographic information systems (GISs) are useful tools for organizing spatial data, implementing models in a spatial context, and presenting the results as maps. They are particularly useful in planning training and testing programs that must be distributed on a facility so as to allow enough space to complete the mission without interfering with other uses of the facility and without using areas that are unsuitable due to mission requirements or risks to natural and cultural resources. In that context, risk models are simply one type of model that may be implemented in the GIS. For that reason, GISs are not discussed further in this report. MERAF may be implemented in a spatially independent manner if the site can be treated as uniform. It may be implemented in a spatially explicit manner but without the use of GIS (e.g., by dividing the site into unit areas that are each treated as uniform), or a GIS may be used.

\subsection{ORGANIZATION OF THE RESEARCH}

This report presents a general conceptual framework of MERAF for assessing the ecological risks from military training and testing programs. MERAF subframeworks are also planned for a set of high priority activities, and an activity-specific subframework has been completed for military aircraft overflights (Efroymson et al. 2000, Efroymson et al. 2001a, Efroymson and Suter 2001). Activityspecific frameworks of MERAF are generic, in the sense that they will explain how to assess the risks from those activities at any place or time. A site-specific framework could also be developed for a specific training or testing facility. It would explain how to assess ecological risks for the set of activities conducted at that site. A site-specific implementation of MERAF has been undertaken for Yuma Proving Ground (Efroymson et al. 2001b). The conceptual framework of MERAF and the overflight framework of MERAF were used.

Users will follow the conceptual framework of MERAF. If they are to perform a risk assessment for a training or testing program at a site, they would determine if a site-specific framework of MERAF has already been developed. If not, they would develop one, as explained in the conceptual framework of MERAF. They would then determine whether activity-specific frameworks of MERAF are available for the activities involved. If some activities do not have activity-specific frameworks of MERAF, they would be developed, once again, following the procedure in the conceptual framework of MERAF. At that point, the assessors would have effectively developed a plan for the assessment and could proceed to perform the assessment using the site-specific and activity-specific frameworks of MERAF. A programmatic assessment that was not associated with a single site would proceed similarly. If the number of sites was small, they could all be assessed as just described for single sites. If the number of sites was large or the sites were unspecified, the assessment could be based on one or more representative sites.

Both site-specific and activity-specific frameworks of MERAF could be developed independently of any specific assessment. In particular, activity-specific frameworks of MERAF for high priority activities should have been developed and disseminated.

\subsection{ORGANIZATION OF THE REPORT}

This report is organized on the basis of linked charts and text. The charts, which are presented at the end of the report, portray the conceptual relationships among tasks, the order in which they are performed, and the flow of information from one task to another. A common numbering system is used to link the charts and text. The overall conceptual framework of MERAF is illustrated in Chart 1 . The major section numbers correspond to box numbers in Chart 1 , and subsections correspond to boxes in charts that elaborate the components of Chart 1.

The components of the activity-specific portion of MERAF (Chart 1, Boxes 4-7) are implemented in two distinct ways. First, for each activity, a generic activity-specific framework of MERAF will be 
developed that contains all of the components necessary to complete the risk assessment for the activity as far as is possible without knowing characteristics of the site or the particular way in which the activity will be performed by a particular training or testing program (Chart $1 \mathrm{~g}$ ). Sections of text and boxes in charts related to the development of a generic framework for an activity have a " $\mathrm{g}$ " following the number. Second, when actually implementing an activity-specific framework of MERAF, the components of the generic framework will be combined with site-specific and program-specific information to actually conduct an assessment. When sections of text and boxes in charts refer to the implementation of the activity-specific frameworks of MERAF, the numbers are followed by an "i."

In addition, MERAF is intended to apply to risk assessments for resource management plans as well as training and testing programs. There are potentially significant advantages to including resource management in the same framework as training and testing activities. First, developing a common set of endpoints ensures that the activities do not have conflicting goals and that the risks are judged in a consistent manner. For example, while a training commander and the natural resources managers may agree on the desirability of a "healthy environment," there could be conflicts if the natural resource managers plan to develop an artificial wetland that would block an important training maneuver corridor. In a case such as this, the common endpoint could be the restoration and maintenance of a more trainingresilient dry-land ecosystem along the corridor. Second, the risks to a resource from training and testing activities depend on the resource management activities. That is, activities that might pose an unacceptable risk when considered in isolation might be acceptable given appropriate resource management activities (e.g., if habitat restoration exceeds habitat destruction). On the other hand, training and testing activities may negate the potential benefits of a given resource management plan.

Three types of risks are associated with resource management plans. First, there is a risk that the plan will not achieve the management goal for the resource and may even have unintended negative effects. Second, resource management activities have effects on species and communities other than the resource being managed and, inevitably, some of these are negative. For example, setting fires to enhance populations of a fire-dependent plant will have negative effects on plants and animals that are not firetolerant or that require the habitat that occurs in the absence of fire. Third, resource management activities may modify the environment in ways that place the military mission at risk. The second and third types of risk are treated the same as a training or testing activity. That is, the fire set for resource management purposes poses risks that are conceptually indistinguishable from fires set by military training or testing. However, risks to the resource from resource management activities are conceptually different at some stages of the assessment process. Where an assessment activity would be performed differently for a resource management assessment, a separate version of the activity is presented in a section with the appropriate number followed by an "r." For example, development of conceptual models is in Sec. 3.6 and the specific instructions for development of a conceptual model for risks to a resource are in Sec. 3.6r.

A few simple conventions are used in these figures and charts. Tasks that are to be performed as part of MERAF and results of those tasks are presented as rectangles. Tasks that are outside the scope of MERAF are presented as rectangles with rounded corners. Decisions are represented as diamonds. Processes are represented as hexagons. All transfers of information are portrayed as an import/export by the downstream chart. Such imports/exports of information from/to a task in another chart are represented by circles. Logical junctions are represented as downward-pointing triangles. 
Organization of the report:

Type of framework

Generic activity-specific framework (does not contain site-specific or program-specific information)

Implementation of actual activity-specific framework tasks (contains site-specific and program-specific information)

Activity-specific framework for resource management purposes
Identification of respective text section

$g$ (e.g., " $4.1 g ")$

$i$ (e.g., "4.3i)

$r$ (e.g., "3.6r")
Identification of respective chart title or section within a chart

$g$ (e.g., "Chart 1g")

$i$ (e.g., " $4 i$ " in Chart 1)

$r$ (e.g., "Chart 4.3r") 


\section{RISK MANAGER AND RISK ASSESSOR}

\subsection{ROLES AND RESPONSIBILITIES}

The primary purpose of performing ecological risk assessments is to provide information needed for a decision. Many of the decisions made in the problem formulation phase involve values rather than facts and, therefore, are policy judgments rather than scientific decisions. These involve questions of what should be protected, what future scenarios are relevant, and what expressions of risk are useful for the decision.

A "risk manager" refers to a function rather than a job title. Within DoD, the risk manager function may reside in a number of places and levels: at the installation or range with the natural or cultural resources managers, with those involved in military operations and logistics activities, with engineers, master planners, and others. Higher-level, more generalized decisions regarding risk must be made at the level of major commands and engineering field divisions, and at the level of the military Services and DoD. In general, the remainder of this report will refer to the "hands-on," onsite risk manager as the "DoD manager." Higher-level DoD personnel, less closely involved with the process, are referred to as "decision makers," simply for convenience of terminology.

The party that conducts the risk assessment is the "risk assessor." The EPA risk assessment framework and the National Academy of Science's guidance upon which it is based, assume that the risk manager and the risk assessor are embodied in different people with different job categories. This may or may not always be the case within the DoD. The actual ecological risk assessment may indeed be performed by a contractor, Service laboratory, or other entity separate from the risk manager. But there may be times when assessment of risk — especially the broader, screening-level of assessment—may be performed by the same person or the same office that is responsible for the decisionmaking. In such cases, the risk manager and risk assessor will be one and the same. The roles or functions themselves are different, however, and the distinction is important for purposes of understanding MERAF. Thus, in this report, a distinction is made between the functions of risk manager and risk assessor, even if these functions may, at times, be embodied in the same person or the same office. One purpose of this report is to help ensure effective communication between DoD personnel and any contractors involved, who may be unfamiliar with each others' work contexts and terminologies.

The risk manager will ascertain which parties are, or need to be, involved in decisions concerning how a training or testing activity will be carried out and to obtain the input of those individuals. Within DoD, broad policy goals are generally set by decisionmakers at the DoD or Services level, and often involve input from specialized organizations, such as laboratories, environmental centers, or policy centers. Often, these decisionmakers are responding to policies set in Congress. The Services generally provide somewhat more detailed guidelines and regulations to facilitate compliance with the policies; however, these are still quite general in scope. The people at the installations, ranges, and other areas where training and testing occur refine and interpret the broad regulations and guidelines into more specific objectives. At this level, the risk manager will provide statements about goals for the particular site or may simply rely on generic policies for site management. The risk manager's function, within DoD, is therefore spread up and down the different levels of management and decisionmaking, and occurs with military, natural/cultural resource management, engineering, master planning, and other domains of activity.

As the process of risk assessment begins, the risk manager, and/or the risk assessors under direction of the manager, should attempt to contact all relevant personnel who are responsible for any aspects of ecological risk management at the site. Risk assessors should obtain as much clarification as possible of the bases for their decisionmaking and the parameters of each of their missions. In the absence of such contracts, risk managers and assessors should look for precedents that would indicate what sorts of ecological issues and evidence have been sufficiently compelling to lead to modification of 
a training or testing program in the past, and which issues and evidence have not. Without input from all decisionmakers involved in the installation/range activities, the assessment process is likely to overlook important aspects of data and context that should be part of the risk assessment.

All parties actively involved should collaborate in the problem formulation. The EPA has developed a procedure for performing this activity at contaminated sites called the Data Quality Objectives (DQO) process, which is the primary operational innovation of their Superfund Accelerated Cleanup Model (SACM) (Blacker and Goodman 1994a,b; EPA 1994). The process involves one or more meetings, each of which may take more than a day. If multiple risk managers are involved, or if non-DoD stakeholders are included in the process, a professional facilitator can be helpful. The DQO process is not directly applicable to the DoD's need for training and testing risk assessments, but the concept of bringing the relevant parties together for a structured process of problem formulation could be quite useful.

\subsection{THE DoD RISK MANAGER'S RESPONSIBILITIES}

This section addresses the following responsibilities of the risk manager in the risk assessment process: policies and legal constraints, management goals, mission goals, decisions to be made, and expectations of the risk assessment.

\subsubsection{Policies and Legal Constraints}

There are many DoD policies that the risk manager may need to apply to the risk assessment process. The most significant of these is NEPA (40 CFR 1500), which forms the basis for protection of the environment. DoD Instruction (DoDI) 4715.3 (1996) prescribes procedures for integrated management of natural and cultural resources on property under DoD control. A 1994 DUSD(ES) memorandum, subject: Implementation of Ecosystem Management in the DoD, states, in part, "Public involvement, communication, and incorporation of public needs and desires into management decisions will be emphasized..." Several implications may follow from such a proclamation: 1) The realistic risk from military mission activities, including newly proposed activities, must be known and, preferably, quantified. 2) The public must accurately perceive this risk in relation to existing activities and other, nonmilitary risks within the adjacent areas. 3) The assessment of risk prepared by the military must be supportable and must be based on principles shared by civilian sector scientists and appropriate regulatory agencies. However, strict compliance with these implications may be prohibitively difficult or impossible for DoD managers to achieve in any realistic way. If these actions are achievable, the cost and time to provide this information is usually considered prohibitive, and only extremely high profile actions usually receive this level of consideration. As history tells us, even this level of detail does not regularly achieve the degree of public consensus suggested by the policy guidance.

\subsubsection{Management Goals}

The setting of management goals occurs in a hierarchical framework. Goals are influenced by policies set at the national level, then the DoD level, the Services, and the command structures below the Services level, down to the level of the installation or field activity, and the organizational levels within the installation. All along the way, military policies and requirements will feed into and affect environmental policies and requirements, and vice-versa. For long-term, overarching goals, the process tends to be more structured, and may include requirements such as an EIS. For more immediate, day-today decisions, the process tends to be less structured and vary considerably by location and organizational level.

Generally speaking, personnel responsible for making and acting on environmental decisions and plans at the military installations cannot do so independently, in a vacuum. Different offices and different personnel are responsible for different aspects of using and managing the environment. For example, the actual "users" are the military personnel who are training or testing in that environment. A number of 
decisionmakers are involved in decisions about this use, potentially including the post commander and the commanders of any offsite troops or other personnel using the site; range and/or flight control officers, operations and logistics personnel, military police, and others. Then, there are decisionmakers among those whose role is to manage and maintain the training or testing facility, including engineers, master planners, and environmental /natural/cultural resources personnel. Finally, there are influences from outside the military facility which can affect decisions about environmental use and management, including federal, state and local policies and influential interests, as well as the interests and needs of neighboring landowners and dwellers.

Each of these parties may have different management goals in mind, at least with respect to shortterm actions. Over the long-term, there is likely to be broader agreement with stated high-level policy goals consisting of "long-term sustainable facilities for military training and testing." The challenge is to achieve the goal of long-term sustainability of both the ecosystem and of options for military use, simultaneously supporting a satisfactory level of training and testing in the short-term, while operating in a resource-constrained environment.

Clearly, this is a tradeoff situation within which all those involved in decisionmaking must operate. None of the goals - environmental or military, short term or long term - can be perfectly satisfied, without unacceptable damage to the other goals. Nor are the resources available to perfectly satisfy any of the goals. To deal effectively with various tradeoffs and constraints, all decisionmaking parties need to be aware of the risks involved in all aspects of the use and management of the training/testing ranges. Ideally, management goals are set through a process of coordination among wellinformed parties representing all decisionmaking "players" among users and managers of the military land, air, and water ranges. It is important that these goals be developed within a context of risk awareness and risk-based management.

Following are management goals that are generally applicable:

Military Mission Goals

- Achieve and sustain military readiness

- Adequately test military equipment and materiel
Environmental Management Goals

- Comply with laws and regulations

- Fulfill stewardship responsibilities

\section{Joint-Interest Management Goals}

- Maintain the environmental infrastructure needed to support military training and testing over the long term

- Find ways to enhance / improve military training and testing by creative environmental management, and to enhance / improve environmental conditions by beneficial adaptations of training and testing procedures.

To achieve these environmental management goals and joint-interest management goals, more concrete and specific goals need to be set. Following are some examples of these:

- Maintain viable populations of threatened / endangered species, based on what is known or theorized about necessary population numbers, habitat sizes and characteristics.

- Decrease accidental take of organisms of concern by a given percentage over a given period of time, using stated methods for doing so.

- Reduce erosion and sedimentation by a given percentage of current levels, and restore vegetation cover on eroded areas.

\subsubsection{Mission Goals}

The basic military mission is very clear: Readiness. All personnel in all Services must be ready to undertake whatever actions are needed, anytime and anywhere. Each serviceman or woman must 
know what to do and how to do it, which means that they must be well-trained and practiced in all their required skills. In addition, personnel must have the necessary equipment and supplies, including weapons and ordnance materiel, which must undergo a rigorous process of development and testing before putting them into operational use. To support mission goals, facilities must be available for individual-level basic training, small-unit training, specialized training, and full-scale force-on-force maneuver training. Realistic maneuver training requires large amounts of space, whether on land, in the air or the sea - and this space must extend not only two-dimensionally across a land or water surface, but three-dimensionally into the air and the depths of the ocean. The same is true of test procedures.

To meet their mission goals, the military services must have at their disposal areas and facilities of a size and nature suitable to support such training and testing. Short-term necessity to accomplish immediate training and testing requirements can often conflict with goals of environmental management. Yet, in the long term, the necessity to sustain the available training and testing areas for ongoing use by the military means that sound environmental management of training and testing areas must be undertaken. Such management is necessary in order to support ranges that are usable, realistic, safe to use, and that comply with legislative and other political and public interest requirements. Thus, military mission goals must take into account both short-term needs for ongoing training and testing, as well as long-term investment in the "infrastructure" - the environment—which supports such training and testing.

\subsubsection{Decisions To Be Made}

The types of decisions about ecosystem management and natural and cultural resources are complex and are made at several different organizational and political levels. Only a few examples of the types of decisions can be given here. One example is the scope of management planning and actions: will the focus be on single (threatened or endangered) species, or on broader aspects of biodiversity and ecosystem management? Will ecological management target only onsite problems, within the boundaries of the installation or range, or will a regional approach be taken, bringing in outside stakeholders and issues of regional concern? What kinds of management actions, and which resources or environmental values will be given priority under constraints of funding, personnel, and time? Since the latter resources are becoming more constrained, such decisions are critical.

Mandates for environmental management are complex, and include local installation mandates, local public ordinances and public pressure, state and federal laws and regulations, and requirements to coordinate with other agencies, Native American tribes, and other organizations. Occasionally, some of these requirements may conflict with each other. In many cases, no formal arrangements are made at any level to ensure that financial and workforce resources are available to meet the requirements. The managers responsible for compliance with these requirements must therefore make difficult decisions about how the requirements can feasibly be achieved.

How can ecosystem management be effectively coordinated with the military mission, at each location: what scientific and military information is needed to do this, what coordination of decision and data management systems is needed, how shall the different organizational units involved in use and management of the ranges and installations communicate and coordinate effectively? The timing and location of ecosystem management actions is often critical, such as timing for prescribed burns, for reseeding, for surveys of biological populations, and so forth. Often the best time to perform a management action for one purpose is the worst time to perform it with respect to a different ecological management goal, and these must be balanced out. Added to the challenge is the need to coordinate the timing of management actions with military use requirements, and with the timing of the government fiscal cycle. All of these involve difficult and complex decisionmaking.

At management levels above the installation, decisions must be made about allocation of financial and workforce resources regarding environmental management at many installations, whose needs must be balanced against each other. Policy decisions about the environment must be made at different organizational levels and levels of detail. The policies must then be resourced, implemented, and 
oversight provided, which require managerial decisions and processes in place. Decisions relating to military doctrine, force structure, and spatial distribution of military units and functions all have effects on military use of the environment, which create new contexts for decisionmaking among environmental managers. Although this is just a brief sampling of the types of decisions that must be made, it is intended to give some indication of the complexity of decisionmaking required on a regular — often daily — basis.

\subsubsection{Types of Decisions}

Management decisions can be made comparatively or noncomparatively. The manager should be aware of the need for comparative decisions in most cases that arise.

\subsection{Comparative Decisions}

The need to compare one goal with another, and decide between them, can come into play under the following kinds of circumstances:

- Constrained resources. Limited funds, personnel, time, equipment, and other resources mean that not all desired management actions can be taken; some must be chosen and others rejected.

- Inherent conflicts between management goals. For example, improving habitat quality for one endangered species may decrease habitat quality for a second endangered species. Managing for a single endangered species may conflict with managing to promote overall biological diversity (species richness). Maximizing income from natural resources through hunting or out-leasing can impact ecosystem balances. The most desired military use of a given space may be strongly detrimental to the most highly valued environmental characteristics of that space, and vice versa.

- Limited opportunities. For example, military users may agree that they can afford to close down one part of a training or testing range for a limited time to allow for rehabilitation. Which part to rehabilitate must be chosen by comparing both environmental parameters and military-use parameters of the candidate sections. Alternatively, there may be several management actions needed on the site, but it can only be closed for long enough to implement one of the actions. Which shall it be?

- Multiple choices are possible. Realignment of a military mission, for example, offers the opportunity to compare types of training or testing activities with respect to their relative impact on the environmental characteristics of the location in question, with the hope that this information will play a role in the realignment decision. Another example is the selection of one among various methods or techniques for accomplishing a chosen management action.

- Possible results or outcomes of proposed decisions are highly variable or uncertain. In such cases, it is valuable to envision several alternatives, including the standard "no action" alternative, and attempt to estimate and compare the degree of risk, costs, and benefits of each alternative.

- Assessment of alternative actions is required. This would be the case where an action falls under NEPA provisions that require an EIS.

The instances given above are likely to occur frequently for DoD decisionmakers; therefore, a method of comparative decisionmaking should be adopted.

\subsection{Noncomparative Decisions}

In some cases, decisions may be made without undertaking a comparative exercise. Following are circumstances where this type of decision could arise.

- Determining whether a specific plan is acceptable according to set criteria. A goal has been set, and the purpose is to determine whether a given course of action will achieve that goal with an 
acceptable degree of risk. The purpose is not to decide between alternative goals or courses of action, but to evaluate the planned action against a given set of criteria.

- A direct requirement may exist to implement a decision that was made outside the purview of the risk assessment process. This can result from military or political orders given within the complex, hierarchical environment of the DoD and the political process, at either high or relatively low levels of the organization. In such a case, there is no need to undertake a comparison to make the decision, since it has already been made. However, a noncomparative risk assessment should still be undertaken if possible, to provide important information for the implementation and ongoing management of the decision. This kind of circumstance can also occur due to stringent regulatory requirements that do not allow room for compared alternatives to given compliance actions.

- An absolute evaluation of a single decision is needed in order to form a basis for deciding whether compared decision making should be pursued. It may be necessary to evaluate potential decisions noncomparatively first to provide a basis for compared decisionmaking as the next step of the process.

\subsubsection{Expectations of the Risk Assessment}

To meet multiple challenges and requirements, DoD managers need better delineation of risks. Understanding effects at an ecosystem level is complex at best. Application of an ecological risk assessment process can assist decisionmakers in several ways. Primarily, the results of the assessment at least should qualitatively describe, and, ideally should quantify the level of risk to the specified resource expected from actions occurring at the predicted extent, intensity, and duration. This specific risk-based information can be balanced with risks to other objectives to assist the manager in making better decisions.

Even if the risk assessment result is not quantitative, the step-by-step identifications and analyses required to conduct the assessment help to extract the most critical elements from the complex ecological problem, thus clarifying, if not simplifying, it. This process begins with identifying specific components of the assessment in the problem formulation step. For example, problem formulation includes clear specification of management goals; specifically what needs to be protected and for what reasons. In addition, it is necessary to identify critical ecological endpoints that can indicate effects on the resource that needs to be protected. This step reduces the ecological complexity of the problem, at least in terms of the number of elements that must be included in the assessment.

Risk assessment ideally takes into account the risks to all of the identified important values. In the cases under discussion here, this means the risks to ecosystems and their components, as well as risks to the military mission. 


\section{PROGRAMMATIC PROBLEM FORMULATION}

The problem formulation phase is a planning process intended to ensure that the risk assessment is defensible and useful. It defines the goals of the assessment, describes the environmental setting, describes the activities to be assessed and their potential environmental consequences, selects endpoints, develops a conceptual model, and develops an analysis plan. This phase covers characteristics of the site and of the overall training or testing program to be assessed. Issues specific to individual activities are in the activity-specific problem formulations (Sec. 4). This section is primarily concerned with assessments of training and testing programs, since those are the most common programmatic assessment problems. In other cases, risk assessments may be performed for natural resource management plans. Such assessments use the same general logic but with different emphases and, in some components, different methods. Those differences are pointed out below.

\subsection{ASSESSMENT GOALS}

Management goals address the desired outcomes of management actions. Assessment goals define the aims of the risk assessment itself, for evaluating risks associated with the current state of the problem and with the actions that might be taken to achieve the management goals. The risk assessment is one among the set of actions needed to reach the management goals, thus it is clear that assessment goals must be based upon management goals. The more specific the goal, the easier it will be to conduct the assessment, and the more useful the results will be in supporting a management decision.

The assessment results should include specific information to support management decisions. This may include quantitative and/or qualitative risk estimates, and explanatory text and graphics geared to the technical proficiency of all decisionmakers involved in the process. Depending on the stated goals for the assessment, the results must include a sufficient analysis of alternatives to support selection of a preferred alternative, such as selection of a particular site or performance of an activity in a certain manner. Table 1 shows example risk assessment goals based on example management goals.

The set of questions that follow are intended to assist in setting the assessment goals.

- What are the specific management goals? Risk assessment goals should match management goals point by point, so the assessment will be a useful and practical tool for developing specific recommendations for achieving management goals. Thus, if management goals include the protection of two endangered species in a given area of land on a training range, then the assessment should provide specific information about risks to those species and that area of land, in a form that can easily be used to make concrete management decisions.

- Who are the parties who must be satisfied with the risk assessment? As far as possible, assessment goals should be formulated with the input of these parties, whether that input is directly and personally expressed, or whether it is contained in regulations, memoranda of agreement, policy statements, or other important guidelines. Examples of interested parties could include:

- military operations personnel whose responsibilities could be affected by the decision

- DoD officials responsible for ensuring adherence to policies

- local public and officials at locations where the decisions will be implemented

- Native American tribal governments

- judicial courts

- regulatory agencies at the federal or state levels, such as the Fish and Wildlife Service or the EPA

- military leaders responsible for actions implemented under their jurisdiction

- environmental advocacy and other interest groups

- scientific experts

- state or federal legislators. 
Table 1. Example ecological risk assessment goals based on given management goals.

\begin{tabular}{|c|c|}
\hline Management Goal & Assessment Goal \\
\hline To protect threatened and endangered species. & $\begin{array}{l}\text { Quantify risks to threatened and endangered } \\
\text { populations from all activities. }\end{array}$ \\
\hline $\begin{array}{l}\text { To choose among alternative flight paths for a } \\
\text { new type of overflight training activity at a base } \\
\text { where multiple overflight activities exist. }\end{array}$ & $\begin{array}{l}\text { Rank alternatives based on mostly qualitative } \\
\text { estimates of risk. } \\
\text { AND/OR } \\
\text { Quantify relative magnitude and probability of } \\
\text { effects of different stressors on various ecological } \\
\text { receptors. } \\
\text { AND/OR } \\
\text { Quantify total risk from current and proposed fly- } \\
\text { over activities, using one or more alternative } \\
\text { assumptions. }\end{array}$ \\
\hline $\begin{array}{l}\text { To define boundaries for a sea range off the coast } \\
\text { of the United States, given a total area x and other } \\
\text { training needs. }\end{array}$ & $\begin{array}{l}\text { Quantify and compare total risks for various } \\
\text { alternatives. }\end{array}$ \\
\hline To close down a base. & $\begin{array}{l}\text { Quantify the risks from activities associated with } \\
\text { the closure. } \\
\text { AND/OR } \\
\text { Compare the total risks associated with the base } \\
\text { closure with those that were associated with } \\
\text { training and testing activities on the base. }\end{array}$ \\
\hline To construct a new heliport. & $\begin{array}{l}\text { Quantify the erosion and other risks associated } \\
\text { with takeoffs and landings of helicopters. } \\
\text { AND/OR } \\
\text { Quantify the risks after mitigation. }\end{array}$ \\
\hline
\end{tabular}

- What questions must the risk assessment address? What are the concerns of the interested parties? The assessment must address the concerns by answering specific questions as well as possible. It can be challenging to elicit and formulate questions and concerns associated with a management decision, since they may not always be clearly stated by those who hold the concerns. If this is not done, however, then the decision may be called into question or even legally challenged, resulting in large costs of time and money, perhaps lost opportunities, and negative impacts on ongoing relations between DoD, other agencies, and the public.

- Are the management decisions comparative or noncomparative in nature? (These types of decisions are discussed in Section 2.2.4.1.)

- If the decisions are comparative, is it sufficient to rank the alternatives? Or is it necessary to estimate the relative magnitudes and probabilities of the risk or the risk reduction associated with each alternative? This will depend on the issues related to the decisions, and the persons and interests that must be satisfied by the decisions. One of the management goals may involve satisfying a regulatory requirement with quantitative compliance metrics, such as maintaining a given number of mated pairs of a threatened species, or achieving a measurable degree of water quality in a heavily sedimented stream. In such cases, it may be necessary to estimate quantitatively the magnitudes and probabilities of the risk associated with each alternative so as to compare them with the requirements. For other, more qualitatively measured management goals, it may be sufficient simply to rank the alternatives. An example of a comparison matrix for ranked alternatives is given in Table 2. 
Table 2. Comparative Effects Analysis Matrix ${ }^{a}$

\begin{tabular}{||c|c|c|c|c|c|c|c||}
\hline Alternative & Land Use & Air & Water & Noise & Soil & Economic & Etc. \\
\hline 1 & $\mathrm{H}$ & $\mathrm{M}$ & $\mathrm{M}$ & $\mathrm{M}$ & $\mathrm{H}$ & $\mathrm{L}$ & - \\
\hline 2 & $\mathrm{M}$ & $\mathrm{M}$ & $\mathrm{L}$ & $\mathrm{M}$ & $\mathrm{M}$ & $\mathrm{H}$ & - \\
\hline 3 & $\mathrm{~L}$ & $\mathrm{M}$ & $\mathrm{M}$ & $\mathrm{L}$ & $\mathrm{L}$ & $\mathrm{L}$ & - \\
\hline 4 & $\mathrm{~L}$ & $\mathrm{M}$ & $\mathrm{H}$ & $\mathrm{H}$ & $\mathrm{H}$ & $\mathrm{M}$ & - \\
\hline
\end{tabular}

${ }^{\mathrm{a}} \mathrm{H}=$ High impact, $\mathrm{M}=$ Medium impact, $\mathrm{L}=$ Low impact

- If the decision is noncomparative, is it sufficient to estimate a qualitative degree of risk, or is there a need to establish more precisely the magnitude and probability of the risks? This depends, again, on the circumstances of the decision. What are the criteria for deciding "go/no-go" decisions? Is it acceptable to decide on the basis of "high or low" risk? Or are there set criteria or metrics against which the results of management decisions must be measured? For example, a memorandum of agreement might have been established with the Fish and Wildlife Service that committed to increasing the habitat of an endangered species to a certain acreage, or the commitment might involve reducing accidental take of marine mammals during testing and training by a certain percentage. Is it desirable to try to predict the likely risks of an action so that mitigation measures can be planned for? DoD budget cycles often require that expenditures be planned several years in advance, so it is advisable to anticipate risks and plan for remedial measures needed as the result of management actions. These considerations should be factored in while the goals of the assessment are being laid out.

- Are the risks of the training or testing activities to be assessed alone, or will the risk and risk reduction of mitigation measures or remedial actions also be factored in? It may be advisable to evaluate risks associated with remedial actions. Different remedial actions will mitigate risks from the training and testing activities to different degrees. In addition, remedial actions may present unintended new risks. Hence, it may be necessary to identify a combination of training or testing activities and remedial actions that result in the least net risk to the environment.

- How will the assessment deal with matters such as uncertainty, lack of data, lack of metrics and standards? What degree of accuracy and certainty is acceptable in the assessment? Uncertainty should be explicitly addressed as part of assessment goal formulation. How much data can or must be collected for this specific assessment, as opposed to relying on existing data and estimates? What restrictions are constraints of time, funding, personnel, information, and other resources likely to place on the risk assessment, and how will these be handled to make the best use of available resources? Data required to generate a quantitative result may not always be available. Depending upon the extent of data required, the criticality of the quantification of the decision, the time requirements, and financial resources available, it may or may not be possible to collect those data for the risk assessment. The quality of the result may thus be affected.

- What is a realistic timeframe for achieving the stated goals of the assessment? If the assessment must be completed in less than a realistic amount of time, then the goals will need to be reformulated to take this time-constraint into account. Particularly for programmatic-level decisions, time allowable to generate results from a risk assessment often may be short. In such cases, it is necessary to provide the best possible risk assessment within the required time. A qualitative result as input to the decision is better than no assessment. But, decisionmakers must be made aware up front, during the problem formulation and goal-setting stages, that severe time constraints will result in less than an ideal risk assessment process, and the assessment goals need to reflect this. 
- The risk assessment, including the selection of goals, must be well-documented. The assessment process and documentation must be able to stand up to scrutiny by judicial courts and other parties that may inquire about the adequacy of the risk assessment process.

The assessment goals and parameters should be clearly formulated to give practical guidance for conducting the risk assessment. Where applicable, the criteria, standards, or metrics that must be met should be delineated. Anticipated constraints on the assessment process should be identified, and ways of addressing these constraints should be built into the assessment goals and parameters.

\subsection{ACTIVITIES TO BE ASSESSED}

Military training and testing programs are designed to fulfill identified requirements of the military mission. These programs are generally comprised of specific activities that interact to accomplish the identified mission. As an example, a training program in land-based countermine operations would include activities such as intelligence, detection, breaching, and passage of minefields. To formulate the problem and conduct a risk assessment at the programmatic level, the component activities of the program and their key characteristics must be identified.

\subsubsection{Defining and Describing the Activities}

An activity is defined as a standard procedure for accomplishing a discrete task. The procedure is repeated over time and at different places to accomplish the training or testing task with different military units. Activities comprise elements described in field manuals and training circulars, and may be components of mission-essential task lists (METLs). Alternatively, an assessment may be conducted on a nonmilitary program, such as soil erosion control. An erosion control program could include activities such as leveling, terracing, constructing checkdams, revegetation, and constructing sediment traps or settling ponds.

To begin the programmatic level assessment, both the program and the component activities need to be clearly identified and defined. For military activities, training doctrine, field manuals, and documentation of standard training and testing procedures will be helpful resources for defining and describing programs and their component activities.

\subsubsection{Frequency and Intensity of the Activities}

At the programmatic level, certain features of the activities must be identified as part of the assessment. Of particular importance is a description of the general frequency of each activity and the intensity of the activities. Intensity is measured as units of activity per unit space per unit time. For example, the intensity of aircraft overflights along a specific Military Training Route (MTR) would be described by stating the number of aircraft flying during a day or other period of time, along with the area of the MTR. More detailed guidance for activity description and analysis is presented in Sec. 4 .

\subsubsection{Are Activity-Specific Frameworks Available?}

As part of programmatic problem formulation, the risk assessor must identify whether activityspecific risk assessment frameworks are already available for each of the component activities of the program. The development of activity-specific frameworks is described in Section 4. If activity-specific frameworks are not available, they will need to be developed or estimated in order to conduct a complete assessment. 


\subsection{ENVIRONMENTAL DESCRIPTION}

Risk assessment must be based, among other things, on knowledge of the baseline conditions existing at a site. The $\mathrm{DoD}$ is in the process of developing baseline data for its installations, ranges, and other areas of use concerning natural and cultural resources, environmental contamination, and other aspects of the environment that are relevant to natural and cultural resources management. Many DoD installations and ranges have at least basic information about these parameters, such as data about soils, elevation, vegetation, water quality, endangered species, and archaeological and historical sites. In many cases, these databases are computerized in a GIS or other computerized database. However, these data may vary considerably in quality, geographic scale, completeness of coverage, level of detail, and year of collection. Thus, the degree of additional data collection needed for ecological risk assessment at a given DoD location could vary from "very little" to "extensive," depending on the site, the activity, and the types of risk and risk endpoints being examined. Data availability and sources for environmental descriptions are discussed in Chapter 5 and several Appendices of Reinbold et al. (1997).

Several sources are particularly useful in providing information on the environmental setting. Data and inventories on natural resources can be obtained from the U.S. Geological Survey (USGS), U.S. Forest Service (USFS), U.S. Natural Resources Conservation Service (NRCS), U.S. and State Fish and Wildlife Service (FWS), among others. The USGS has information on land surfaces, precipitation measurements, and water quality. Topographic maps for the project site areas at the 1:24,000 (7.5 minute) scale can also be obtained from USGS. Other pertinent data available from USGS include Land Use and Land Cover maps, Landsat Satellite Imagery, energy source maps, water flow and water quality data from streams and rivers, land ownership maps, and aerial photographs. The National Oceanic and Atmospheric Administration (NOAA) and National Air and Space Administration (NASA) provide additional data about ocean water and subsurface land characteristics, and climatic factors. The USFS has information on forested areas, logging practices, and timber sales. The FWS, the National Marine Fisheries Service, and the State Heritage databases developed by The Nature Conservancy can provide information on listed species, habitat requirements, and guidelines for protection of threatened or endangered species. The NRCS can provide soil surveys, information on soil characteristics, and soil series classification. Through the National Park Service, one can access the National Archaeological Database and the National Historic Sites database to obtain information on sites and maps of cultural value. State Historic Preservation Offices also have information about cultural resources, as may individual Native American tribal governments. Appendix H of the report by Reinbold et al. (1997) provides World Wide Web (WWW) addresses of many of the agencies mentioned above, as well as additional federal and state agencies. City, county, and regional planning agencies should be able to provide land-use plans and maps as well as demographic and economic information.

\subsection{PROGRAMMATIC SPATIAL AND TEMPORAL BOUNDS}

The scope of the assessment must be specified on spatial and temporal scales. That is, one must determine what area during what time period will be the subject of the assessment. Three types of bounds are defined:

Type of bounds

Core area and time

Influence area and time

Endpoint area and time

\section{$\underline{\text { Description }}$}

Areas and time periods in which direct exposure to training and testing activities occur.

Areas and time periods in which indirect consequences of training and testing activities occur.

Areas and time periods in which the endpoint species or communities are present. 
It is also important to consider whether prior training or testing programs need to be considered along with the proposed program. In many cases, the environment will not have recovered from prior training or testing activities before a new program begins. In such cases, the simplest approach is to treat the condition of the environment at the beginning of the program as baseline and estimate risks of incremental effects. In that case, bounding of the assessment is not affected by prior activities. In some cases, however, it may be desirable to assess the cumulative risks in an integrated manner. This is usually the case when risks result from complex or nonlinear processes of recovery and succession. In those cases, temporal bounds may extend back to the beginning of use of the site for a particular type of training or testing activity. In effect, the definition of the program is expanded to include the historic use of the range for training and testing.

Note that, in addition to these areas and times in which risks must be assessed, other areas and times may need to be considered in the assessment. In some cases, forcing functions or drivers of the risk models may be derived from conditions outside of the area or time being assessed. For example, when assessing risks to freshwater fish living within a military facility, areas in the upper watershed determine water quantity and quality even though these areas may not lie in the training or testing range, and may not be influenced by training or testing activities.

\subsubsection{Combine Bounds of Activities}

The spatial and temporal bounds for the programmatic problem formulation are defined by combining the spatial and temporal bounds for all of the individual activities (Sec. 4.3). That is, the area assessed is the area: 1) directly exposed to any of the constituent activities (the core area), 2) indirectly exposed to the activities (influence area), or 3 ) in which an endpoint occurs that overlaps with the core or influence areas (endpoint area). These areas may bear different relationships to each other depending on their relative size and arrangement in space (Fig. 3.4-1). The time period assessed is the time from the beginning of the earliest activity to the end of the last activity (core time) and the time for the receptors to recover from all direct and indirect effects (influence time). In some cases, as with migratory species, periods of time when endpoints are present or absent must be included in the formulation (endpoint time).

\subsubsection{Define the Core Area}

The core area is simply the area encompassing all of the activities of the program. It can be defined by map or GIS overlays. If the program includes spatially distinct activities, then the core area will include multiple unit areas. If the assessment addresses the risks to a particular endpoint (e.g., redcockaded woodpeckers or desert tortoises), then multiple types of core and influence areas may exist (e.g., one for the training ranges and one for areas subject to habitat enhancement) (Fig. 3.4-1a).

\subsubsection{Define the Core Time}

The core time is simply the time from the beginning of the first activity of a program to completion of the last activity. It is defined by a programmatic time line.

\subsubsection{Define the Influence Area}

The area influenced by a program is the sum of the areas outside the core area that are indirectly affected by the activities. It can be defined by map or GIS overlays.

\subsubsection{Define the Influence Time}

The time influenced by a program is the time from the end of program activities to the recovery of all endpoint receptors. 


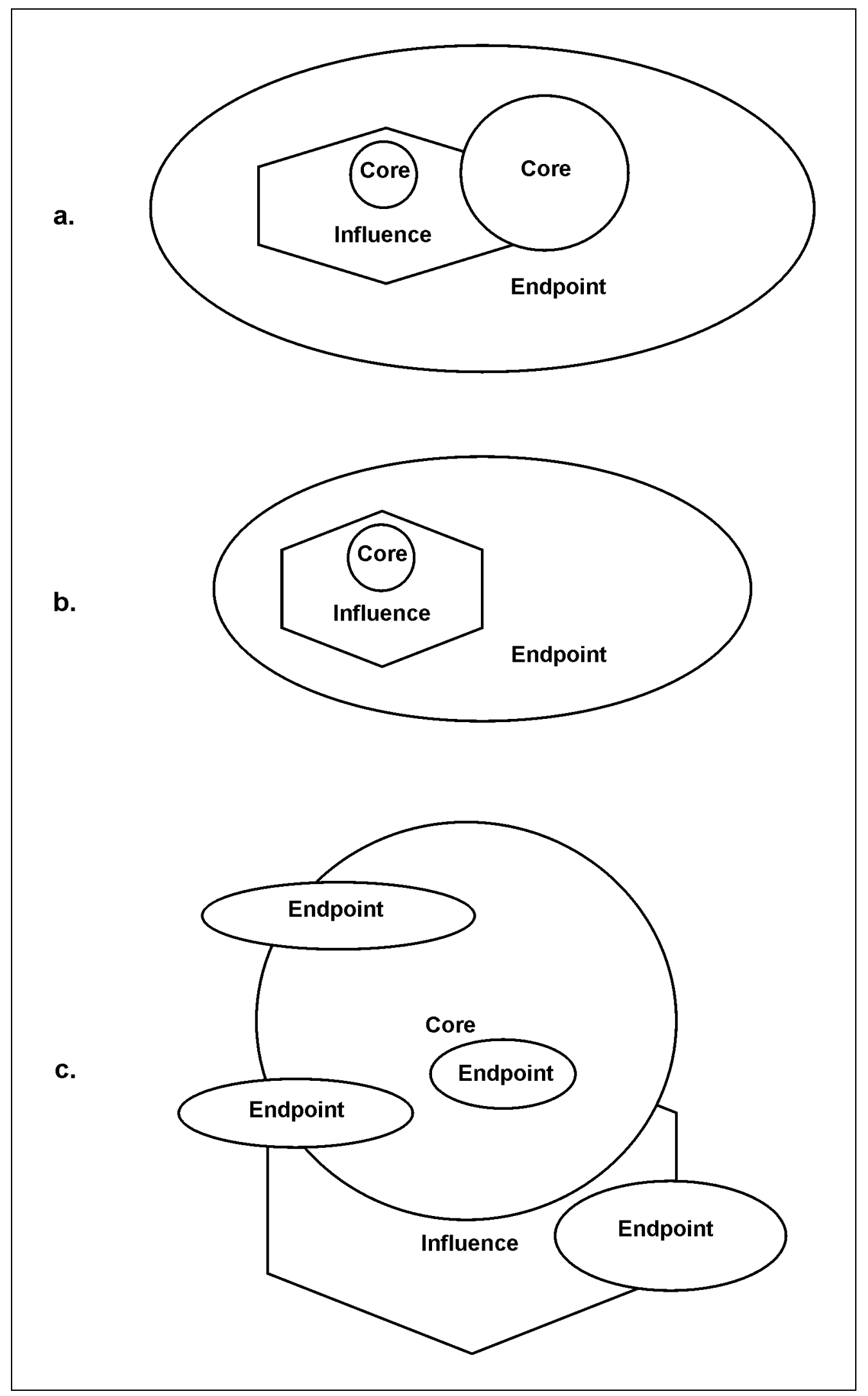

Figure 3.4-1. Examples of possible relationships among core, influence, and endpoint areas. 


\subsubsection{Does the Core Area Change with Time?}

During a brief program, the entire core area is effectively in use for the entire time. That is, although the entire area is not literally in use for the entire time, the movement of activities within the area does not have important implications for the risks to natural resources. For example, wildlife populations cannot shift from one habitat to another, and the phenology of the plant communities does not change from one area to another during the time that the program is in operation. However, if a program will be carried out over a long time (e.g., multiple seasons) and the use of different areas will shift over those long time periods, then that change in area with time must be defined and incorporated into the assessment. The definition of "long time" as used in the preceding sentence depends on the temporal dynamics of the processes involved.

Considerations include the rate of recovery, the rate of dispersal, and the rates of seasonal changes in the natural and physical characteristics, such as soil moisture. Finally, if recovery processes are important and if recovery proceeds at significantly different rates in different areas, that may cause the core area to change over time.

\subsubsection{Does the Influence Area Change With Time?}

Influence areas change with time primarily due to dispersal of contaminants, degradation processes, and recovery processes. For example, silt from erosion of devegetated areas will contaminate an increasingly large area of streambed. Following recovery of vegetation, the input of silt will decline and periods of high flow will remove silt from the stream faster than it is being added. Eventually, the silt is diluted into a large enough volume of water (e.g., Fort Knox silt in the Ohio River) that the influence can be assumed to be negligible. As with the core area, the assessor must judge whether the time dimension is significant, based on the temporal dynamics of exposure relative to the rate of response of the endpoint receptors.

\subsubsection{Define Area as a Function of Time}

The simplest function relating area to time is binary; either the activity is occurring in an area or it is not. Binary functions are applicable to many direct effects for those cases in which activities are rotated among areas. Continuous functions are applicable to cases such as the spread of a contaminant into new areas or an increase in the portion of the area used. For example, if vehicles use a route through an area until it becomes impassible, then use a new route until it becomes impassible, etc., then the area used increases with time. Note that, while some of these functions can be quantified during the problem formulation (e.g., changes in the areas used), others cannot. For example, the change in area disturbed by tanks in the example above would not be quantified until the risk characterization is completed. However, it is important during the problem formulation to specify that there is a relationship between space and time that will be addressed by the assessment.

\subsubsection{Define Endpoint Area and Time}

The bounds identified thus far are bounds on the areas either directly or indirectly influenced by the activities of the program. However, depending on the definitions of the endpoints (Sec. 3.5), the area and time that must be considered may extend to areas that are not affected by the activities, but are part of the definition of an assessment endpoint. For example, if an assessment is concerned with the effects of tank maneuvers on an endangered lizard, the direct effects are limited to areas in which the tanks maneuver, and the indirect effects extend to areas in which the lizard may be behaviorally affected by the noise and vibrations or areas in which habitat is damaged by erosion, fires, etc. However, if the endpoint is "probability of extinction of the lizard" or some other property of the species, the area assessed must include the range of the species (Fig. 3.4-1b). Similarly, the time assessed must be extended from the duration of the program and its influence on the environment to the entire planning horizon of the 
recovery program for the species. Alternatively, the endpoint entity may appear in multiple small areas (e.g., individual wetlands) within the core and influence areas (Fig. 3.4-1c). The spatial and temporal scope of each assessment endpoint is defined in Sec. 3.5.7.3. This step integrates spatial and temporal scope with the military activity and the geography of the site to define the overall scope of the assessment.

Note that many endpoints are not defined in a way that requires consideration of larger areas or times. In particular, for abundant and widely distributed species or communities, training and testing activities will not influence the properties of the species or community type as a whole. In those cases, the endpoint will typically be defined in terms of the population or community on the facility and there is no need to consider areas other than the core and influence areas for the training or testing activities.

The other relevant aspect of the endpoint scale is the minimum area and time that must be affected in order to be potentially significant (Sec. 3.5.7.3). If the areas in which the endpoint occurs are identified, then the criterion can be refined as follows. If the area of overlap between the endpoint area and the core area or the core area plus influence area (if the endpoint is susceptible to the indirect exposures) is smaller than the minimum area for the endpoint, the endpoint should be dropped from the assessment. Similarly, if the temporal overlap between the occurrence of an endpoint and the core time or time of influence (if the endpoint is susceptible to the indirect exposures) is shorter than the minimum time for the endpoint, then that endpoint should be dropped from the assessment.

\subsubsection{Define the Programmatic Bounds}

The spatial and temporal bounds of the program include the areas and times in which activities are carried out (core areas and times), areas and times in which the activities indirectly affect the environment (influence areas and times), and areas and times defined by the assessment endpoint (endpoint areas and times). These bounds should not be simply combined because they are treated differently in the analysis due to their different exposures.

\subsubsection{Define Unit Areas and Unit Times}

If the area to be assessed is large and either the environment or the activities are spatially heterogeneous, it is appropriate to divide the area into units within which risks are estimated. The purpose is to create areas within which the exposure-response dynamics are sufficiently uniform that one can characterize the risks. For example, forests and grasslands may be assessed separately and each community type might be further divided into impact areas and non-impact areas. For upland ecosystems, units would simply be areas of land with the prescribed characteristics. For streams and floodplains, the units would be reaches. Reaches are units that are bounded by tributaries, structures such as dams, modifications such as rip-rap or channelization, or by changes in use, such as reaches that are exposed to vehicular crossings.

Unit times are defined when different activities are conducted in different periods or when important environmental characteristics change over the time period being assessed. As with spatial units, the temporal units should be sufficiently uniform that data can be averaged over the time period and the risks estimated. For example, a two- or four-seasonal division of the year is often a useful time-unit when dealing with biological organisms. However, the schedule of training/testing activities might, for example, change on a monthly basis. A common time-unit must be chosen to best facilitate the goals of the analysis.

\subsection{ENDPOINT SELECTION}

In the programmatic problem formulation, assessment endpoints are selected that are appropriate to the site, the specific activities to be assessed, and the service's policy goals. Assessment endpoints are 
explicit expressions of the environmental values that are to be protected (Suter 1989; EPA 1992). Endpoint selection identifies which environmental entities are sufficiently valued to potentially change a management decision, are particularly ecologically important and susceptible to the proposed activities, and are practical for assessment. It then defines the endpoint in terms of a specific entity (species, assemblage, etc.), a property of that entity (abundance, number of species, etc.), and a scale (area and time).

Criteria for selection of assessment endpoints have been published in the EPA framework. They are:

Environmental policy goals and societal values - Because the risk to assessment endpoints is the basis for decision-making, it is important that the endpoints reflect the policy goals and societal values that the DoD and its sub-units are expected to protect or enhance.

Ecological relevance (importance) - Entities and properties that are significant determinants of ecosystemic properties are more worthy of consideration than those that could be added or removed without significant system-level consequences. Examples include a keystone predator species and the process of primary production. to the exposure.

Susceptibility - Susceptible entities are those that are potentially highly exposed and responsive

In addition, two other criteria must be added to the MERAF structure:

Mission relevance - Properties of the environment that are needed for performance of a training and testing mission must be included as assessment endpoints. In general, these relate to providing an environment that is realistic and not hazardous to the training and testing personnel.

Cultural relevance - Preservation of cultural resources, including historic places, is an important goal of the DoD. In many cases, the natural context of a cultural resource is an important component of its cultural value. In such cases, these characteristics of the natural resources should be identified as potential assessment endpoints.

The endpoint selection process for the programmatic problem formulation is diagramed in Chart 3.5. The input to this process consists of the site description and the $\mathrm{DoD}$ manager's value judgments. The environmental description should provide a list of candidate assessment endpoints that should be filtered through management's criteria for assigning societal value.

\subsubsection{Candidate Assessment Endpoints}

To define assessment endpoints using the criteria, it is necessary to develop a list of candidate environmental and cultural entities. Sources of such candidates include prior environmental assessments, resource inventories, and ad hoc environmental and cultural surveys.

\subsubsection{Prior environmental assessments}

The first source of such entities is precedents from prior assessments. If prior ecological risk assessments have been performed for the facility, these endpoints are obvious candidates for new assessments. Other sorts of assessments are not likely to have endpoints as clearly defined as those in risk assessments. A review of prior NEPA EISs and assessments, however, can provide a list of environmental and cultural resources that have been important to prior decisions. In a few cases, Natural Resource Damage Assessments will have been performed for the site. Natural resources defined as injured in those assessments should also be candidate assessment endpoints. 


\subsubsection{Resource inventories}

A number of data inventories may be available at DoD sites. Installations generally have Master Plans, available from the Master Planning Office within the Directorate of Public Works (DPW) or from departments of engineering and housing or real property. Range Control can provide information on training activities, schedules, rotations (annual and weekend training dates), type of units, number of vehicles, kind of equipment, etc. The Natural Resource office can provide data, inventories, and maps on natural resources, often including forestry inventories. This office will sometimes have floristic inventories, and generally has a list of threatened and endangered species. Most Army installations have Land Condition Trend Analysis plot data. Often, useful data can be obtained from the State Heritage databases of species developed by The Nature Conservancy. The National Marine Fisheries Service may have relevant species data and inventories for coastal and marine areas. Many of the newer inventories and databases are available in GIS format. Overall, there may be considerable variation among DoD installations and ranges concerning the type of inventories available, their quality, geographic scale, completeness of coverage, level of detail, and their recency. The Services are actively pursuing the improvement of their natural and cultural resources inventories, and one may expect to see ongoing improvement in the quality and coverage of these inventories in the relatively near future.

\subsubsection{Ad hoc surveys}

When natural and cultural resources have not been inventoried for a range or facility, then the general data obtained as described in Sec. 3.3 should be used to identify candidate assessment endpoints. In general, it is appropriate to divide the resources into terrestrial, aquatic, wetland, and cultural. The natural resource entities should then be identified as candidate species, candidate assemblages (e.g., small mammals, fishes, benthic invertebrates), and candidate ecosystems. The cultural resources should be classified as archeological, architectural, and other.

\subsubsection{Compilation of candidates}

This step is a simple collation and elimination of duplicates. Near duplicates (e.g., fish and game fish) should not be eliminated at this point, because they may have different susceptibilities, different societal values, etc.

\subsubsection{Ecological Importance Criteria}

Entities that should be considered when selecting assessment endpoints because of their ecological relevance include:

Major contributors to energy or nutrient dynamics - For example, the dominant plants are important to both energy and nutrient dynamics in all ecosystems.

Providers of important habitat structure - Some organisms, because of their physical structure, increase the physical diversity of habitats or provide shelter or cover where it would not otherwise be available (e.g., trees and sea grasses).

Modifiers of the physical structure of the environment - Some organisms, such as beavers by building dams, earthworms by mixing the surface soil, and alligators by excavating ponds, modify the physical structure of the environment in ways that are important to many other species.

Regulators of physical or biogeochemical processes - Some species play important roles in controlling ecological processes. For example, wild cherries rapidly sprouted on clear-cut sites in the Hubbard Brook Experimental Forest, NH, and played an important role in retaining nutrients on the site. 
Consumers that regulate the relative abundance of their prey species - Some species, termed keystone species, control the relative abundance of species in a community through selective predation (either carnivory or herbivory).

In addition to these generic criteria, some site-specific criteria may be applied. These would be based on knowledge of the ecological structure and function of the site. In general, these criteria are the most difficult to apply. There is often little information available to judge the importance of species beyond relative abundance and broad generalizations.

\subsubsection{Policy and Societal Values Criteria}

Although the selection of endpoints on the basis of policy and societal values criteria may be done $a d h o c$, for the sake of consistency and transparency, criteria should be developed for selecting valued components. Possible criteria for selection include:

Threatened and endangered species - Species listed by the federal or state governments as threatened or endangered are legally protected from any "taking." This protection is prima facie evidence of societal value. This value is based on the goals of preserving biodiversity and preventing extinction, as well as public concern for particular species.

Other rare species - Other species that are rare nationally or locally have been listed by government agencies and private entities. These include candidate threatened and endangered species. Although these species are not legally protected, they may be considered to have value in terms of preservation of biodiversity, as well as public concern for particular species.

Game and commercial species - The societal value of species that are harvested for commercial, recreational, or subsistence purposes is manifest.

Species with local significance - Species with particular religious, aesthetic, or cultural value should be included.

Wetlands - Wetlands have been afforded legal protections based on their habitat value to wetland-dependent species and their role in hydrologic regulation and nutrient cycling.

Rare ecosystem types - Rare ecosystem types, such as barrens and old-growth forests, have value in terms of preservation of biodiversity, as well as public concern for particular ecosystems.

Because of the nature of these criteria, it is particularly important to involve other agencies, and stakeholders, as appropriate, and to consult DoD and Service policies during this step in the process.

\subsubsection{Mission Criteria and Ecological Constraints}

There are several primary considerations concerning the interrelationships between natural resources/ecosystems and the military training and testing missions. One is the provision of a realistic environment, which applies especially for training, but also for testing. Not only do military activities affect the environment; environmental features, conditions, and constraints have important impacts on the quality of military training and testing. Furthermore, a good understanding of the environment-its hazards and its opportunities - is a major and essential aspect of military tactics. The relation between mission criteria on the one hand, and how the natural environment can enhance or detract from meeting these criteria on the other, is an area of knowledge that still needs considerable advancement, through cooperative study and mutual communication by both military experts and natural scientists. 


\subsubsection{Environmental realism}

Effective military training and testing requires that such activities take place in a real-world environment, one which provides as realistic a training or testing experience as possible. Servicemen and women must be trained to understand and use the tactical aspects of the environment, such as concealment, elevation, line of sight, and natural obstacles or protective features of the landscape or the underwater environment.

\subsubsection{Logistics}

Logistical considerations such as trafficability of different soils and landforms are important; vehicle drivers must learn their capabilities and limitations with regard to slopes, mud, sand, snow, inundated areas, visibility and blind spots, and other transportation challenges. Surface and subsurface features of marine and coastal environments also offer logistical challenges, including obstacles, hazards, and visibility affected by water quality. Constraints due to sensitive environments and habitats, on land or water, can create logistical challenges because of the way vehicles and troops must move around and avoid such areas. Damaged environments and environmental constraints can cause increased logistical difficulties for military activities.

\subsubsection{Navigation and intelligence-gathering}

Navigation and intelligence-gathering at sea, on land, and in the air depends, in part, on a good understanding of the natural characteristics of the landscape, and coastal and marine areas. One example is the need to understand the infrared, radar, and sonar signatures of different natural features. Also, visible/measurable features of the landscape and water environment give clues about important, but lessperceptible, characteristics. For example, slight changes in surface water patterns can show an underwater obstacle, and landscape clues can indicate the presence of a dangerous sinkhole or potential land-slip area. Intelligence personnel must gather information not only about human activities in warfare, but also about the environment, so that good tactics can be applied and hazards avoided.

\subsubsection{Military engineering}

Military engineers must be able to recognize and use natural features to provide barriers, bivouacs, assembly points, take-off and landing strips, routes of access, and other services to the warfighter. This must be accomplished in an efficient and problem-free manner, without having problems such as poor drainage, land slippage or subsidence, or many other difficulties that could result from poor understanding and use of the natural landscape.

\subsubsection{Human safety and equipment maintenance}

Other considerations include human safety, and damage, wear and tear on equipment. Badly damaged training areas develop gullies, landslips, unstable slopes, deep ruts, waterlogged areas, sinkholes, precariously balanced trees and boulders, airborne dust and sand, and many other safety hazards. These hazards can and have caused human injuries and deaths, as well as large costs for damage and unnecessary wear and tear on military equipment. Such hazards also detract from the quality of the training exercises by forcing the warfighters to deal with the hazards of the landscape - such as one's vehicle becoming stuck or otherwise disabled - instead of taking part in the actual military exercise.

\subsubsection{Legal and political constraints on use of the environment}

An area where the military mission intersects with ecological considerations concerns the constraints placed on military use of a range — land, air, or sea — because of legal or other sociopolitical sanctions against a given type of use or impact on the environment. The protection of endangered species 
has particularly wide repercussions in terms of constraints it places on military use of land, sea, or airspace. Certain military activities and areas have been delayed, halted, or closed down entirely due to such constraints. Taking a proactive stance with regard to these constraints is far more efficient than trying to undo or live with situations that have reached a precarious or intolerable status. By looking ahead, by developing a good understanding of, and working with, ecosystems and their components on the one hand, and military needs and mission criteria that relate to use of the environment on the other hand, it is often possible to significantly reduce constraints placed on military activities for ecological reasons.

The more a training area is altered from its natural state through ongoing damage and abuse, the less able it is to provide warfighters and support personnel with the opportunity to learn how to interpret and make use of natural landscapes, and how to avoid natural dangers and pitfalls.

\subsubsection{Considerations Regarding Cultural Resources}

This category does not refer to cultural resources per se, which are subject to their own assessments, or to natural resources that have cultural importance in themselves, which are included directly as societally valued resources (Sec. 3.5.3). Rather, it refers to changes in natural resources that could influence the value of cultural resources. For example, a historic site may have greater cultural value if the surrounding ecosystem type is the same as when the historic events occurred. Types of cultural resources that should be considered include:

Archeological sites and entities - Ruins or other artifacts or locations with known or suspected archeological features should be considered.

Architectural resources - Structures that have received national landmark designation or some equivalent designation should be considered.

Natural sites with local cultural significance - Natural sites with particular religious or cultural value should be considered.

Criteria for selection of an ecological endpoint based on cultural values include:

Aesthetic influence - The natural environment visible from the cultural resource site may influence the cultural value of the site.

Historic authenticity - The resemblance of the site to its state at the time the historic events for which it is valued, or at the time when the cultural artifacts were created, may influence its cultural value.

Species associated with the cultural resource - In some cases, particular species are associated with cultural resources. Their presence and abundance may influence the value of the cultural resource.

The collaboration of experts on cultural resources and consultation with representatives of the culture involved (e.g., Native American tribes) must be sought when cultural values are at risk.

\subsubsection{Screening Against Criteria}

The selection of valued candidate endpoints may be performed in two ways. If the criteria for endpoint selection have been developed (Sec. 3.5.2 through 3.5.5), this step consists of applying the criteria to each of the entities identified in Sec. 3.5.1. Otherwise, the selection must be performed ad hoc by the DoD manager, staff, and any stakeholders that the manager chooses to include in the process. If endpoint selection is performed ad hoc, it is important for participants to specify why something is societally valued, ecologically important, or relevant to the mission. This information is critical to the endpoint definition (Sec. 3.5.7). 
Susceptibility is a function of the degree to which a receptor is exposed and its inherent sensitivity. Hence, it is specific to the stressor imposed by an activity. In MERAF, the susceptibility of potential receptors is reviewed for each distinct activity (Sec. 4.2). Hence, at this stage in the programmatic problem formulation, the assessors would refer to the framework for the activity to determine what is known about susceptibility. For example, if the activity is tank training, one might find that wetland plants are susceptible, and, if the activity is testing torpedoes, one might find that cetaceans are susceptible. One would then apply these generalizations to the list of candidate endpoints to determine which of the valued or important candidate entities are also susceptible.

If criteria exist, the task consists of 1) evaluating the candidate assessment endpoints (Sec. 3.5.1) with respect to each set of criteria, resulting in lists of susceptible, ecologically important, societally valued, or mission-relevant candidates, and 2) collating the lists and eliminating redundancies. Because the lists will have been developed using different criteria, the same entities may be identified in different terms or aggregated to different degrees. For example, the list may include aquatic vertebrates, the fish community, and individual fish species. The assessors must determine whether these differences reflect real distinctions that must be preserved in the endpoint selection process or whether they are inconsequential and the candidates should, therefore, be combined.

In the authors' experience, ecological risk assessments generally have too many endpoints rather than too few. Large numbers of endpoints result in less effort devoted to accurately and fully assessing the risks to any one. In addition, the reports of assessments with large numbers of endpoints are voluminous and confusing. Hence, the endpoint selection process should result in a short list of endpoints that are truly important to the decision.

\subsubsection{Define Assessment Endpoints}

The assessment endpoints must be defined with sufficient specificity that they can be measured or modeled. This includes defining the entities in terms of their level of organization and scale, and the properties of the entities. These components of the definition are presented sequentially, but they are interdependent.

\subsubsection{Definition of endpoint entities}

The specification of entities provided by the screening against societal values and ecosystem importance is often somewhat vague, and the same entity may be specified in different ways. In particular, it is important to specify the level of biological organization for each endpoint. The level selected should be the level that displays the properties that are to be protected (Sec. 3.5.7.2), but may also be constrained by the spatial and temporal scales. In addition, tradition and precedent play a role. For example, freshwater fishes are usually sampled as an assemblage by netting or electrofishing, and then community properties are calculated (Karr et al. 1986; Plafkin et al. 1989). In contrast, wildlife and marine fishes are traditionally evaluated as individual populations.

Organism Level - In general, protection of individual organisms is appropriate only for threatened and endangered species. However, individuals of other highly valued species may be protected as well.

Population Level - Resource species (e.g., game fish, birds, and mammals; timber trees; etc.) are most appropriately assessed at the population level, as are species that have a particular cultural value or ecological importance specific to the species (e.g., keystone species). Most wildlife endpoints are defined at this level, but there is no reason other than methodological tradition why one might not treat small mammals or birds as a community if none of the component species are particularly valued.

Community Level - Some assemblages are valued as a group, particularly where no one species is more valued than others. Examples include benthic macroinvertebrates and fish. Community-level 
endpoints are preferable when biodiversity concerns are dominant, but are not focused on specific endangered species.

Ecosystem Level - Some ecosystems, such as wetlands, are valued for their properties as ecosystems rather than their composition as communities. Some components of ecosystems are clearly ecologically relevant for their role in ecosystem processes, but have not been valued in the United States for their population or community properties. The soil heterotrophic community is a prominent example. In addition, certain natural environments are valued for training and testing missions because their resemblance to potential battlefields provides realistic tactical training.

\subsubsection{Selection of endpoint properties}

The appropriate properties of the valued and ecologically important entities depend on the ecological level of organization of the entity and the criteria that led to their selection, as described below. These general properties should be selected from, modified, or supplemented for activity-specific assessments as appropriate, based on properties of the stressors associated with the activity.

Organism Level - Individual survivorship and reproductive success are appropriate endpoint properties for endpoints defined at this level. Behavioral properties, particularly avoidance of potential habitat, may also be appropriate.

Population Level - In general, the appropriate endpoint properties for populations of endpoint species are abundance, production, or range.

Community Level - In general, the appropriate properties for endpoint communities are species richness and abundance. The measure of abundance will vary among communities. For example, the abundance of the fish community is determined as numbers of all component species, whereas herbaceous plant community abundance may be expressed as biomass per unit area. Various diversity indices have been used, but they are no more sensitive to toxic effects than species richness, and are less understandable by decision managers and stakeholders.

Ecosystem Level - Specifically protected properties of wetlands in the United States are provision of habitat for wetland dependent species, regulation of hydrology, and retention or cycling of nutrients.

Properties of specific classes of receptors that might be endpoint properties are discussed below.

Soil ecosystem properties - Given the importance of soil as a biogeochemical system supporting all terrestrial life, it would seem obvious that assessment endpoints for contaminated soils should include appropriate soil properties. However, it is not self-evident which properties are appropriate. Many of the properties, such as reduced nutrient availability and changes in the relative abundance of microbial taxa that change in soils following contamination with organic contaminants (e.g., petroleum), result from biodegradation, a desirable process. In other words, many of the changes occur because the contaminant acts as an organic substrate as well as a toxicant. As a result, many of the soil processes and properties that have been proposed as test endpoints would not be appropriate (Health Council of the Netherlands 1991). For example, soil respiration increases as organic chemicals degrade and net nitrogen mineralization is reduced due to immobilization. These effects can mask any toxic effects on mineralization of native organic carbon and nitrogen. In addition, to most decisionmakers and stakeholders, the soil is a black box that is acceptable if it supports plants and animals. Therefore, soil properties have seldom been drivers for decisionmaking relative to release or remediation of contaminants. The effects of physical disturbance of soil are more readily evaluated and the physical form of the soil including ruts, gullies, mud, and dust has obvious effects on the performance of training and testing missions. Soil retention (the inverse of erosion rate), density (affected by compaction), and macro and microstructure (lost by churning, compaction, and erosion) are all potential endpoint properties. 
Plant properties - Plant production is one of the clearest and most generally accepted assessment endpoints for ecological risk assessment. The biological and societal importance of plant production is clear. Also, plants have a scale of exposure that is appropriate to disturbed or contaminated sites because plants do not wander out of the area, and many sites are large enough to encompass a population of herbaceous plants. This immobility makes plants particularly susceptible to physical disturbances. Although plants generally do not appear to be particularly sensitive to soil contaminants, their sensitivity is not well predicted by other receptors, and they are highly sensitive to some chemicals. Although various other properties might be used for the assessment endpoint (e.g., mortality or species richness), the common use of tests of plant growth suggests that production is the appropriate endpoint property for exposure to contaminants. Other properties, such as percent cover or biomass, may be more appropriate for physical disturbances. Plant cover and structure are usually important to training and testing missions.

Properties of soil fauna - Soil invertebrates are ecologically important in terms of soil structure and nutrient cycling, and as food for wildlife. They are potentially sensitive to soil contaminants due to their intimate contact with, and consumption of, the contaminated soil. Because of their low mobility, they have an appropriate scale for many assessments. Their societal significance is less clear. A review of the bases for regulatory decisions by the EPA found that aquatic and benthic invertebrates, fish, birds, mammals, reptiles, amphibians, and plants were considered, but soil invertebrates and microorganisms were not (Troyer and Brody 1994). If the DoD manager chooses to make remedial decisions on the basis of effects on soil invertebrates, they are appropriate assessment endpoint organisms. The appropriate property is less clear. The common use in the United States of earthworm survival, growth, and reproduction as toxicity test endpoints suggests that the assessment endpoint is population abundance or production of earthworms, or of all invertebrates as represented by earthworms. The Dutch have used protection of $95 \%$ of species of soil invertebrates as an endpoint (Van Straalen and Denneman 1989) as well as survival, production, and abundance of earthworms and collembola (Health Council of the Netherlands 1991).

Properties of terrestrial vertebrates - Mammals and birds are commonly used as assessment endpoints for contaminated or disturbed terrestrial sites. Vertebrates in general are less ecologically important than plants, invertebrates, and microbes, however, and their ranges are often inappropriate for the scale of the assessment. That is, all bird populations and many other vertebrate populations have much larger ranges than typical contaminated or disturbed sites. Even individual vertebrates often have ranges much larger than contaminated areas. As a result, the susceptibility of vertebrates is often low if risks are realistically assessed because the exposure is diluted over the entire range of organisms and the effects are diluted over the population range. Shrews and moles are potentially important exceptions because they have relatively small ranges, and they have high dietary and direct exposures to contaminants. Terrestrial salamanders and burrowing anurans and reptiles are also potentially highly sensitive, but their responses to chemical exposures and habitat disturbance are relatively unknown and there are no standard toxicity tests for them. Commonly used endpoint properties for terrestrial vertebrates include the abundance or production of populations.

Properties of aquatic vertebrates - Fish are commonly used as assessment endpoints. Where a particular species is selected because of its commercial or recreational value, production or abundance are appropriate properties. In risk assessments for contaminants, properties of the fish assemblage are commonly used, including number of species and number of individuals. Other aquatic vertebrates including reptiles, amphibians, pinnipeds, and cetaceans have been seldom used in risk assessments, so there is little precedent for selecting properties. Aquatic reptiles and amphibians are generally less abundant than fish, less valued, and more difficult to survey or test. Marine mammals are also less abundant than fish and much more difficult to survey or test, but they have high societal value, due to public concerns. Injury and avoidance behavior of cetaceans have been important endpoint properties for the Navy's assessments of risks from explosions and noise. Properties of those taxa should be selected $a d$ hoc based on the nature of the activity that places them at risk. 
Properties of aquatic invertebrates - Planktonic invertebrates have seldom been used as endpoint entities in ecological risk assessments, but benthic invertebrates are commonly used. Generally, the entity is the entire benthic macroinvertebrate assemblage and the properties are the number of species, number of individuals, or various diversity indices.

Properties of aquatic plants - Aquatic plants are seldom used as endpoint entities, despite their obvious importance. The most commonly used property is production, although community properties have also been used. In estuaries, the abundance of submerged aquatic vegetation has also been a concern because of its importance as habitat structure and as a trophic resource. In freshwater, introduced aquatic plants have been important system stressors and interfere with boat operation, wading, and swimming. For assessments of these risks, biomass and the area occupied by exotic plants are appropriate properties.

\subsubsection{Selection of candidate assessment endpoint scales}

The scale of effects is an important component of a full endpoint definition that is often neglected. The scales of the endpoints, along with the scales of the activities, determine the spatial and temporal bounds on the assessment (Sec. 3.4). In addition, the scales of the endpoints determine the spatial and temporal units within which effects must be estimated by the assessment and detected by any monitoring that is conducted. The following questions must be answered:

What is the total area occupied by the endpoint entity? The answer to this question depends on the exact definition of the entity. For example, if the entity is a species, the area may be the entire range of the species (kit fox), the range of a distinct recognized population of the species (San Joaquin kit fox), the range of a population defined ad hoc on the basis of biology (San Joaquin kit foxes on the Elk Hills and adjoining habitat areas), or the range of a population defined ad hoc on the basis of stewardship responsibilities (San Joaquin kit foxes on the Elk Hills Navel Petroleum Reserve).

For what time is the endpoint present? Most endpoint entities occur continuously on a site, but some are migratory or otherwise transient. For the latter entities, the times when they are present must be identified.

Over what area must the effect occur to be potentially significant? Effects in very small areas are generally inconsequential. On the other hand, defining an effect over too large an area will result in dilution of an important local effect within a large, relatively undisturbed area. This question is closely related to the determination of the area within which the endpoint property is expressed. Variations in the spatial scales of potential endpoint entities can be seen in the following example. 1) The death of an individual of an endangered plant may occur in $1 \mathrm{~m}^{2}$. 2) The abundance of an herbaceous plant population, the plant species richness of the meadow community in which it occurs, and the abundance of a vole population in that meadow may be defined within a hectare. 3) However, the abundance of the red-tailed hawk population that uses that meadow must be defined in an area of at least several square kilometers. Questions to be answered in determining the spatial scale of the endpoint include:

- Over what areas do distinct populations or communities of the endpoint occur (see above)?

- What area is required for individual units of the entity (e.g., territory or home range size)?

- What area, what number of unit areas, or what proportion of the total area occupied by the endpoint must be damaged to potentially affect the valued properties of the endpoint population or community?

- What area of the endpoint must be damaged to potentially affect a mission?

- What area of the endpoint must be damaged to potentially affect a cultural resource?

For what time must an effect occur to be potentially significant? A transient effect will not be sufficient to cause a change in training or testing plans, but the definition of transient is not self-evident. At the other extreme, averaging effects over geological time scales is inappropriate, because all effects are likely to be unimportant at a sufficiently large scale. Hence, a time period must be defined that matters with respect to ecological, cultural, or mission goals. 
The results of this exercise in bounding the endpoint should include:

1. The area occupied by the endpoint entity.

2. A minimum area or proportion of the total area within which an effect is potentially significant to serve as the unit area.

3. The time period during which the endpoint entity is present.

4. A minimum time over which the effect must occur to be potentially significant.

\subsubsection{Review of Assessment Endpoints}

Because of the importance of assessment endpoint selection to the success of an ecological risk assessment, it is important to review the endpoint list before proceeding. Although the criteria for endpoint selection have been in use for several years (Suter 1989), it has become apparent that they have been subject to misunderstanding in practice. Table 3 contains guidance on avoiding common problems in defining assessment endpoints. These rules should be applied to the endpoint list, and the endpoints should be modified to avoid the identified problems.

\section{5r ENDPOINT SELECTION: RESOURCE MANAGEMENT}

Endpoint selection should not be difficult for assessments of risks associated with resource management activities. The resource to be managed (e.g., a deer population) is the endpoint entity, and the management goals define the endpoint properties (e.g., abundance) and scales (e.g., the bounds of the facility). If the management goals are not well defined, however, it may be necessary to clarify them in order to perform the assessment.

Table 3. How to avoid common problems with assessment endpoint definitions identified by the EPA (1998).

1. Ensure that the endpoint is a property rather than a goal (e.g., not "maintain and restore endemic populations").

2. Ensure that the endpoint is clear and operationally definable, not vague (e.g., eelgrass abundance and distribution rather than estuarine integrity).

3. Ensure that the endpoint is a valued property, not simply something related to a valued property (e.g., midge emergence, rather than the production of fish which partially depends on midge production).

4. Ensure that the endpoint is directly or indirectly exposed to the contaminant or disturbance (e.g., do not use fish community as an endpoint when there is no surface water contamination).

5. Ensure that the endpoint is relevant to the site (e.g., the site must offer habitat for the species or community).

6. Ensure that the endpoint has an appropriate scale for the site (e.g., not golden eagles on a $1000 \mathrm{~m}^{2}$ site).

7. Ensure that the value of all entities are sufficiently considered (e.g., do not reject benthic invertebrates at a site where crayfish are harvested).

8. Ensure that the chosen property includes the value of the endpoint entity (e.g., when the community is valued for game fish production, number of fish species is not a sufficient endpoint property).

9. Ensure that the chosen property is sufficiently sensitive to protect the value of the endpoint entity (e.g., when the entity is valued for its production, survival of game fish is not sufficient to protect game fish production). 


\subsection{PROGRAMMATIC CONCEPTUAL MODEL}

Conceptual models are representations of the hypothesis about how effects may be induced as a result of an activity or set of activities. They summarize the results of the problem formulation in terms of cause and effect relationships. Guidance is available for development of conceptual models for ecological risk assessment (Barnthouse and Brown 1994; Suter 1996a). However, that guidance is not adequate for MERAF because it does not adequately account for the potential complexity of training and testing programs, and because it does not provide a means for efficiently generating models.

This introduction presents five components of a strategy for conceptual model development that allows for complexity and is efficient. 1) Make the models explicitly mechanistic. 2) Define the compartments as functional groups. 3) Include the exposure-response relationships. 4) Create hierarchies of detail so that all important processes can be included without creating massive and confusing charts. 5) Create modular components of the model representing the activities to be assessed, influences on the endpoint receptors, and site-specific entities and processes that link activities and receptors. Sections 3.6.1 - 3.6.4 describe the steps in generating a conceptual model for a training or testing program.

\section{STEP 1: MECHANISTIC CONCEPTUAL MODELS}

Representation of mechanisms has not been an important issue for most ecological conceptual models that deal with contaminants. Those conceptual models represent the release of the contaminant, its transport through the environment, and its contact with receptors. Hence, the flow charts used in these conceptual models literally represent the flows of the contaminant from the source to the receptors. This makes the models relatively simple and easy to read (e.g., Fig. 3.6-1). For assessments of training or testing, the conceptual modeling approach must be able to represent contaminant flows, plus a variety of other interactions, including the induction of secondary effects. An approach developed for the EPA is potentially useful (Suter et al. 1994). Rather than showing contaminant flows, the conceptual model diagrams represent cascades of processes, states that result from those processes, processes that result from those states, etc. (Fig. 3.6-2).

Presentation of the conceptual model as cascades of processes and states has three advantages. First, it makes clear to the reader how the components of the system are assumed to be linked. Otherwise, an arrow linking a vegetation box to a tortoise box without an intermediate process might represent a trophic relationship, a habitat relationship, or both. Second, the construction of conceptual models in this manner imparts a useful discipline by forcing the assessors to think through the mechanisms behind the model more clearly than models that simply string compartments together. In addition, creating a mechanistic conceptual model lays the groundwork for the integrated risk characterization that must be based on the mechanisms by which the various agents act on the endpoint receptors (Sec. 9). Finally, a conceptual model in this form provides a basis for quantitatively modeling the system. For the mathematical modeler, the conceptual model shows how the stated variables and forcing functions are to be assembled (Jorgensen 1994).

\section{STEP 2: DEFINITION OF COMPARTMENTS}

Like parameters in a mathematical model, the compartments in a conceptual model may be identified in more than one way. In particular, they may be based on different methods of classification and may be more or less aggregated. For example, a vole may be classified trophically as an herbivore, taxonomically as a rodent, functionally as a burrowing animal, or by a hybrid classification such as the physically and taxonomically defined class, small mammal. Voles may be a compartment, or they may be a member of any of those possible aggregated compartments.

In general, because the conceptual model is a representation of processes that lead to risks, the definition of compartments should be based on functional groups. Functional groups may be defined in two ways (Catovsky 1998). First, they may be defined in terms of the functional response of the group to a prior process. For example, highly mobile animals are functionally distinct in their response to wildfires 
from less mobile organisms that cannot avoid being burned. Second, they may be defined in terms of their functional effect on a subsequent process. For example, herbaceous plants, but not trees or algae, function as input to herbivory by voles. These types of functional groups have been termed functional response groups and functional effects groups (Catovsky 1998).

The position of a compartment in the model determines its type of functional group. An endpoint compartment is clearly a functional response group. In Fig. 3.6-1, for example, the function of insectivorous wildlife is a response to exposures resulting from consumption of herbivorous insects. (Note in Fig. 3.6-1 the useful convention of defining the compartment as a functional group and listing specific endpoint species belonging to the group.) A source compartment is clearly a functional effects group. That is, only sources that affect the receiving system in the same way should be aggregated.

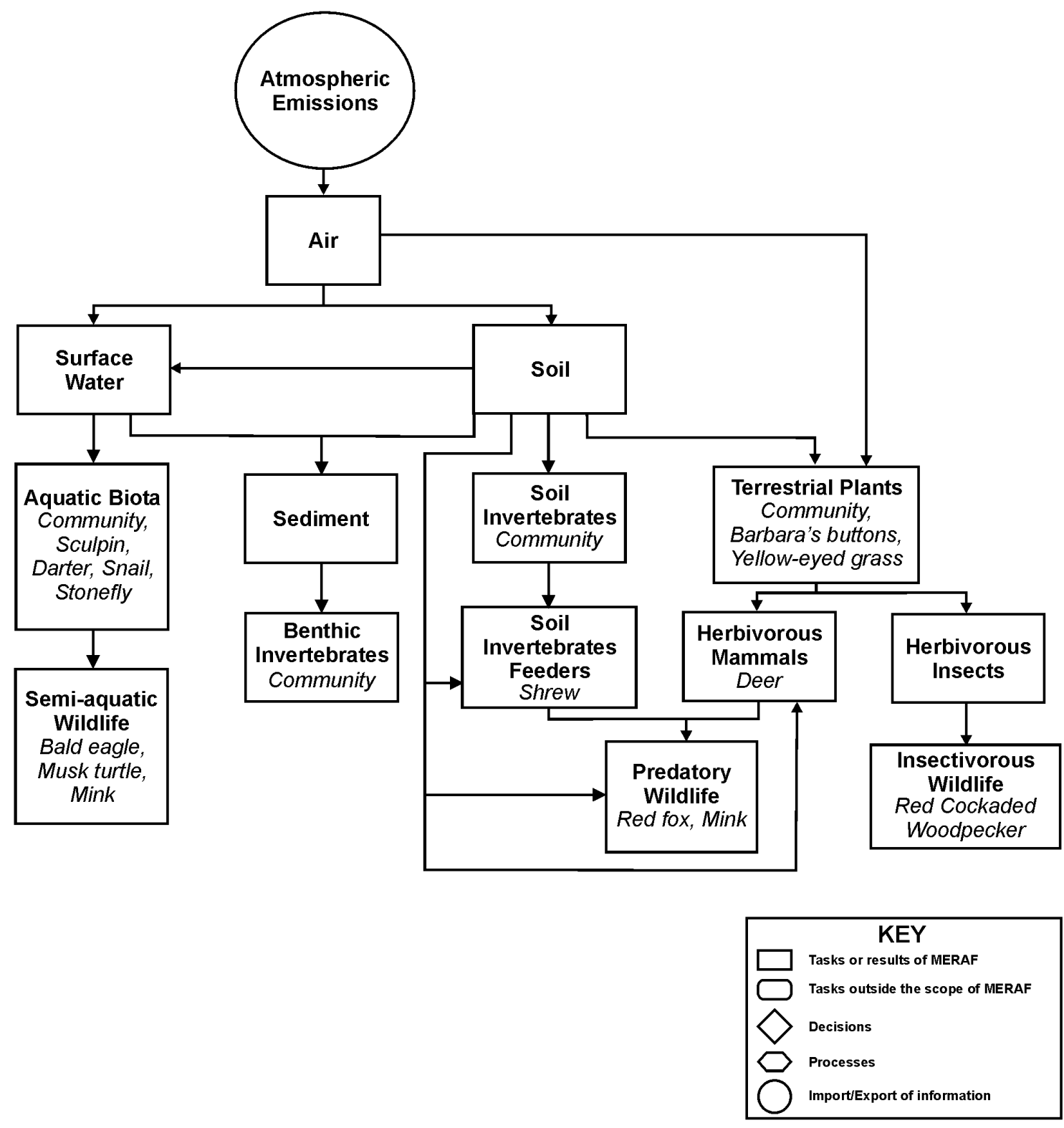

Figure 3.6-1. Example of a conventional conceptual model for exposure to chemicals. The source is a chemical weapons incinerator. 
However, all compartments that are causally intermediate must be both functional response and functional effects groups. That is, they must respond similarly to prior processes that influence them and must affect subsequent processes and states in the same way. In Fig. 3.6-1, for example, a property of the soil invertebrate community is an assessment endpoint so that compartment is a functional response group. In addition, it is a functional effects group with respect to soil invertebrate feeders. Hence, intermediate functional groups must be uniform with respect to both types of functions.

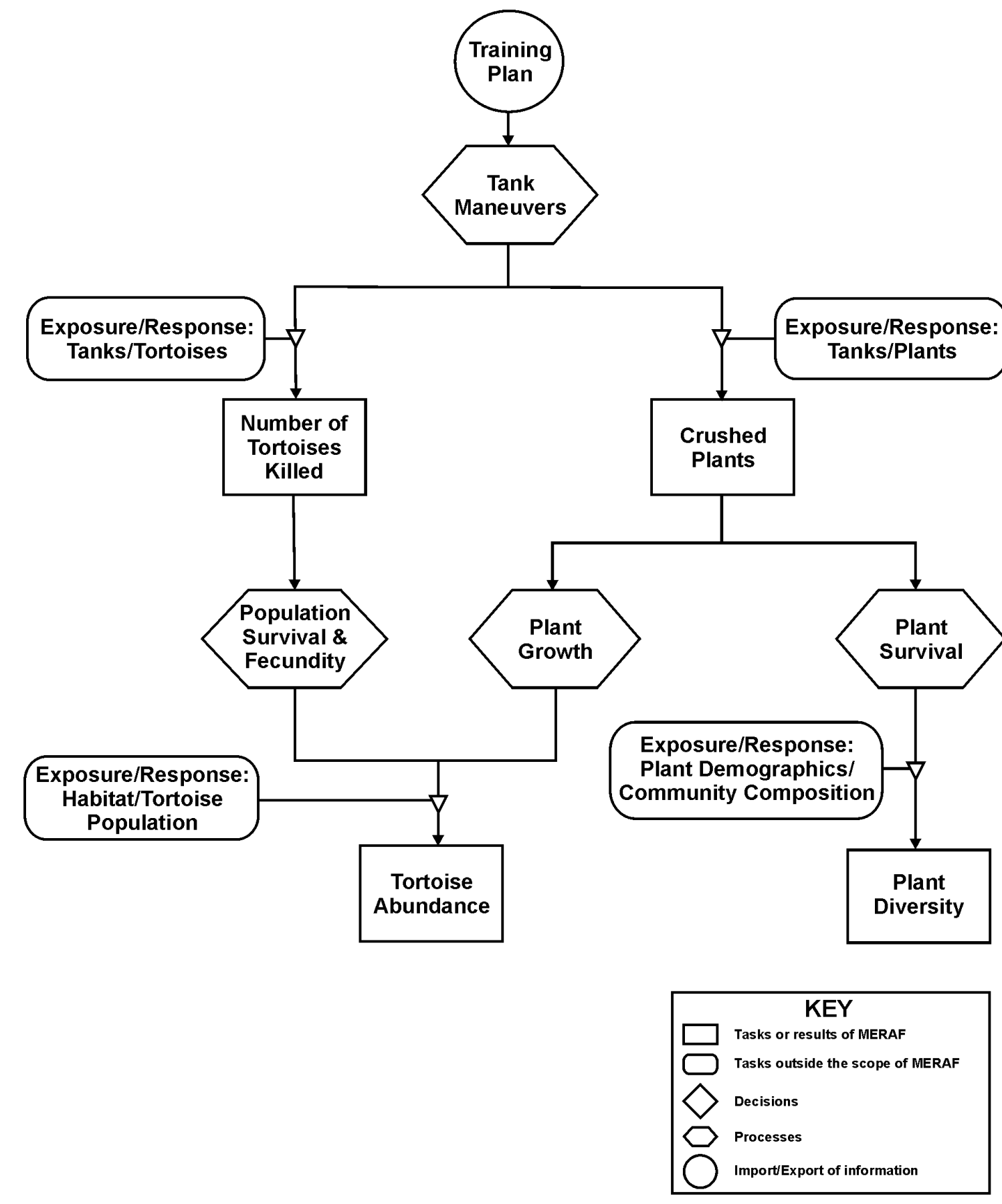

Figure 3.6-2. Hypothetical activity-specific conceptual model for tank maneuvers. 
If compartments are defined as groups with common response or effects functions, the problems of choosing a classification system and level of aggregation can be logically resolved. That is, the correct classification is the highest one in which all members share the pertinent function and the correct level of aggregation.

\section{STEP 3: EXPOSURE-RESPONSE RELATIONSHIPS}

Conventionally, conceptual models are representations of processes by which a release of a contaminant results in exposure of an endpoint receptor. The induction of effects is not included because it is implicitly assumed to occur after the exposure process. When indirect effects are assessed, however, that assumption is inappropriate. This may be because effects on one environmental component affect others (e.g., loss of plant production affects herbivores) or because effects at one organizational level affect others (e.g., death of individuals affects population production). As a result, effects may be induced at multiple points in the conceptual model. To avoid confusion and to clarify the assessment structure for reviewers, it is valuable to incorporate the exposure-response relationships into the conceptual model. One convention for this is shown in Fig. 3.6-2. Exposure-response relationships are portrayed as functions that regulate the influence of an exposure process on a receptor state. This inclusion of exposure-response processes, along with the exposure processes discussed above, makes the conceptual model fully mechanistic.

\section{STEP 4：HIERARCHICAL MODELS}

Conceptual models for assessments that involve multiple activities and endpoint receptors may become so complicated as to be useless as communication tools. While conceptual models that resemble a microprocessor's logic diagram are more confusing than enlightening, it is also inappropriate for the conceptual model not to represent all of the complexity that occurs in the assessment models. The solution to this problem is to create hierarchical sets of conceptual models with each lower level containing representations of the states and processes aggregated at the next higher level.

Consider a relatively simple activity, such as release of smokes. The subsequent state is smoke in the air; the subsequent process is exposure of organisms to the smoke; and the final state is the affected receptor organism (Fig. 3.6-3). This is the most aggregated and simplest form of the conceptual model for risks to wildlife from smoke releases. This model can be elaborated by breaking down any step into a series of more detailed processes and states. For example, the state "smoke in air" could be elaborated as a series of transfers among, losses from, and concentrations of, the smoke's constituents in atmospheric compartments due to advective, convective, and depositional processes. Similarly, the exposure of a receptor, such as a herbivorous mammal to smoke constituents, could be disaggregated into direct inhalation, deposition on vegetation and dietary uptake, and deposition on fur and grooming (Fig. 3.6-4).

\section{STEP 5: MODULAR CONCEPTUAL MODELS}

The modularity of conceptual models results from creating and then combining three types of component conceptual models. First, component models may be created for a site or class of sites at which multiple assessments will be performed. This could be a military training or testing facility or range that will be the site of multiple training or testing programs. The second type of component models is the receptor conceptual model. These are models that represent the influences on standard (common) endpoint receptors. The third type of component models is the generic activity conceptual model for each activity at a site or class of sites that may be assessed. The three types of models are described below. A conceptual model for a program would be created by linking these three types of component models (Fig. 3.6-5).

This modular technique has two potential advantages. First, it provides a means of organizing the generation of conceptual models to reduce the likelihood of missing important aspects of the situation. That is, assessors are less likely to ignore important aspects of any components if they create the overall conceptual model after first individually conceptualizing the activity, site, and receptors. Second, once a 
component conceptual model has been developed, it is available for use as a module in overall conceptual models for other programs at a site, other sites with the same activities, other activities affecting the same receptor, etc. For example, a standard food web model can be a component of all conceptual models for a site or region.

\subsubsection{Obtain or Generate Site Conceptual Models}

General site models show relevant site features of all programs performed on that site. The most obvious example is a hydrologic flow model that shows how dissolved and suspended materials move through and out of the site. An example for the Oak Ridge Reservation is shown in Fig. 3.6-6. An equivalent model for a training and testing facility would show relationships among specific ranges and other use areas. Another example would be a food web that shows how materials move through the biotic components of the site by trophic transfers.

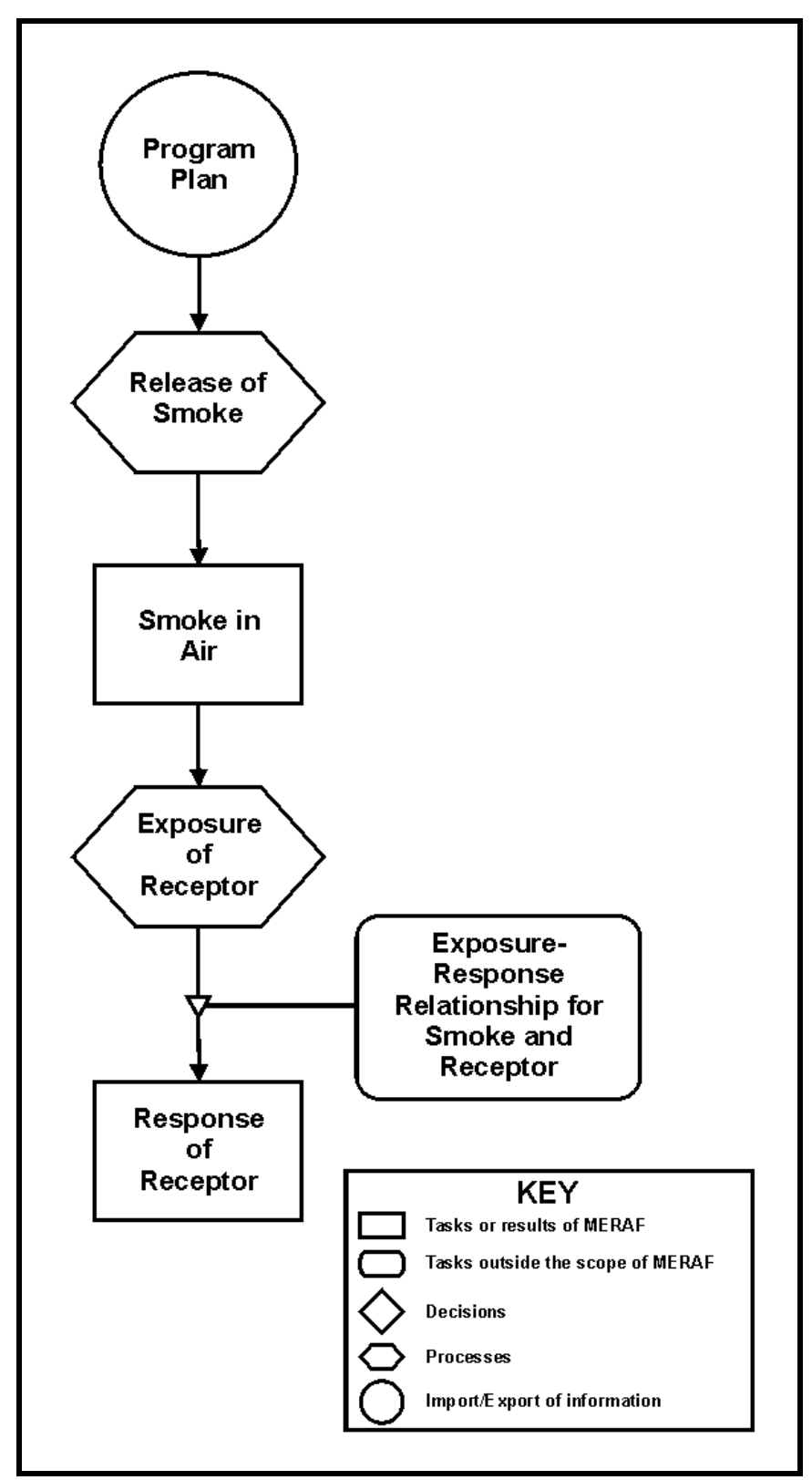

Figure 3.6-3. Hypothetical higher-level conceptual model for release of smokes. 


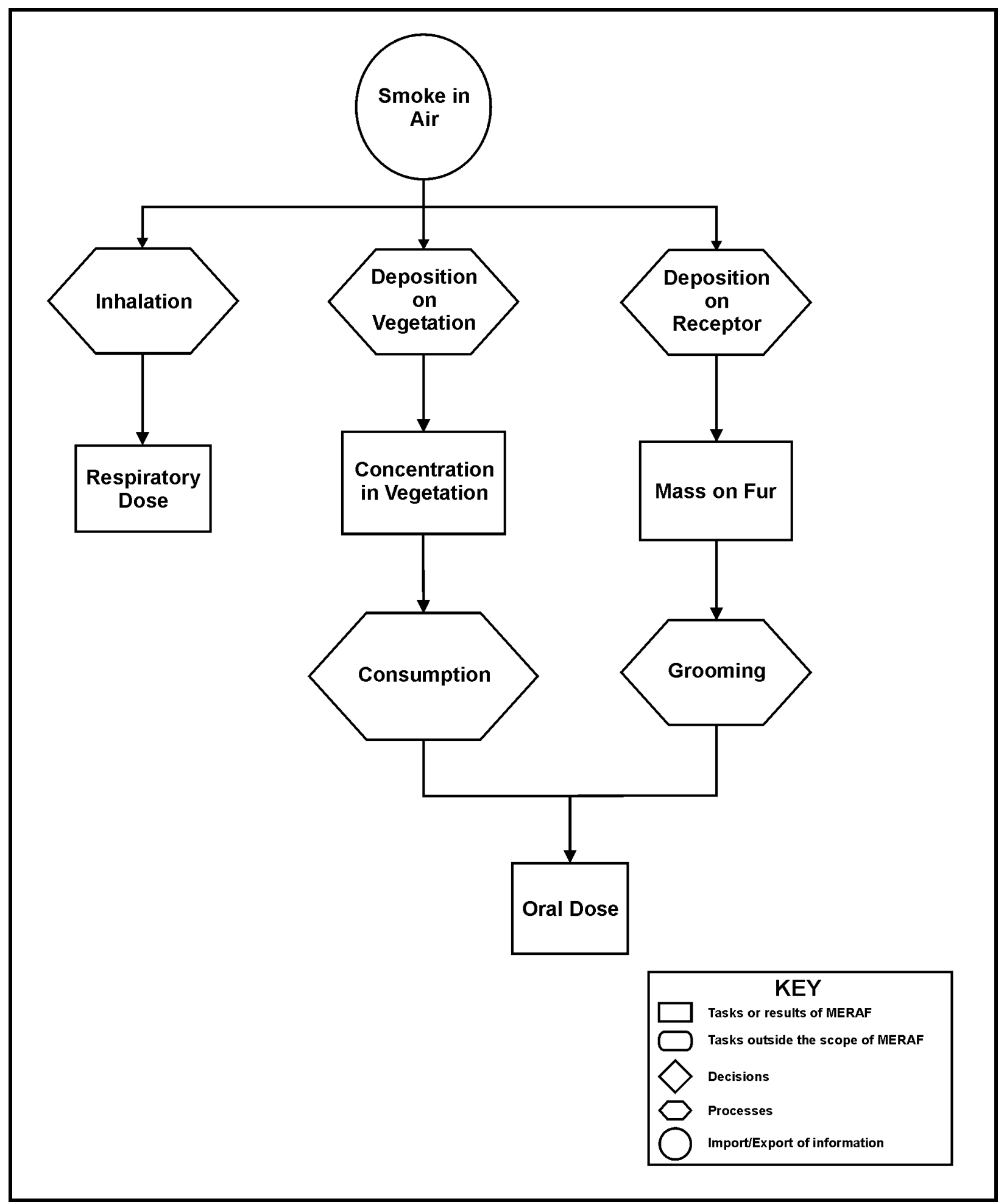

Figure 3.6-4. Hypothetical lower-level conceptual model for release of smokes. 

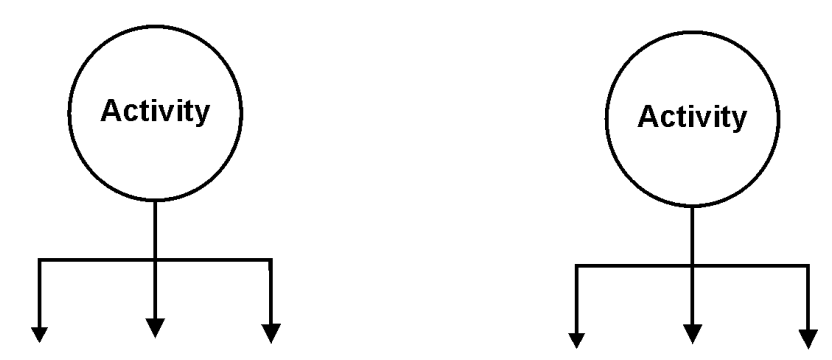

Activity-Specific

Conceptual Models

Consequences

Sources
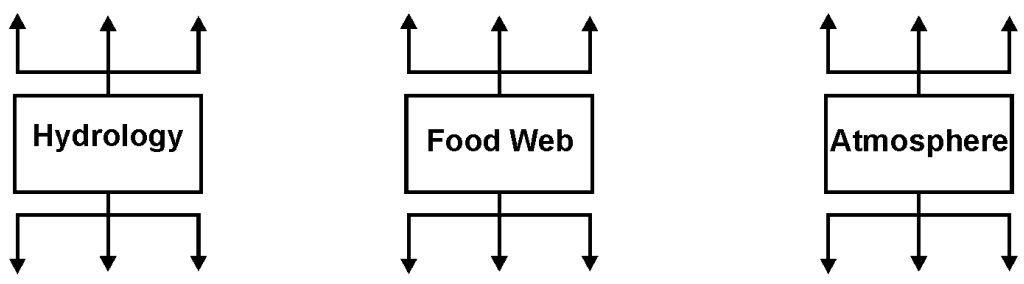

Site-Specific

Conceptual Models

Transport and Consequences

\section{Exposure Routes}
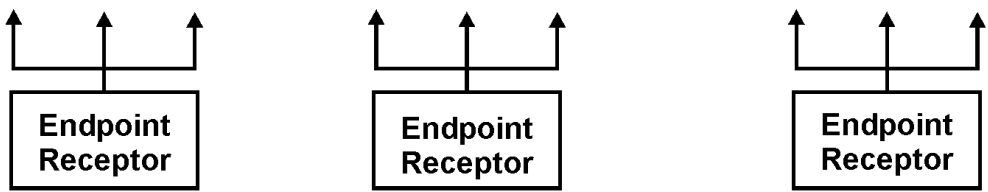

Receptor-Specific Conceptual Models

\begin{tabular}{|l|}
\hline \multicolumn{1}{|c|}{ KEY } \\
\hline Tasks or results of MERAF \\
Tasks outside the scope of MERAF \\
\hline Import/Export of information
\end{tabular}

Figure 3.6-5. Assembly of a programmatic conceptual model from component conceptual models.

\subsubsection{Obtain or Generate Receptor Conceptual Models}

Receptor models show the influences on the properties of endpoint receptors. These would be produced for receptors that are expected to be endpoints for most ecological risk assessments on the site. For example, one would expect that, if red-cockaded woodpeckers were widely distributed on a site, the abundance of that species would be an endpoint for nearly all programmatic risk assessments. In that case, it would be efficient to develop a model of how features of the environment influence red-cockaded woodpecker abundance. Such a model is under development. 


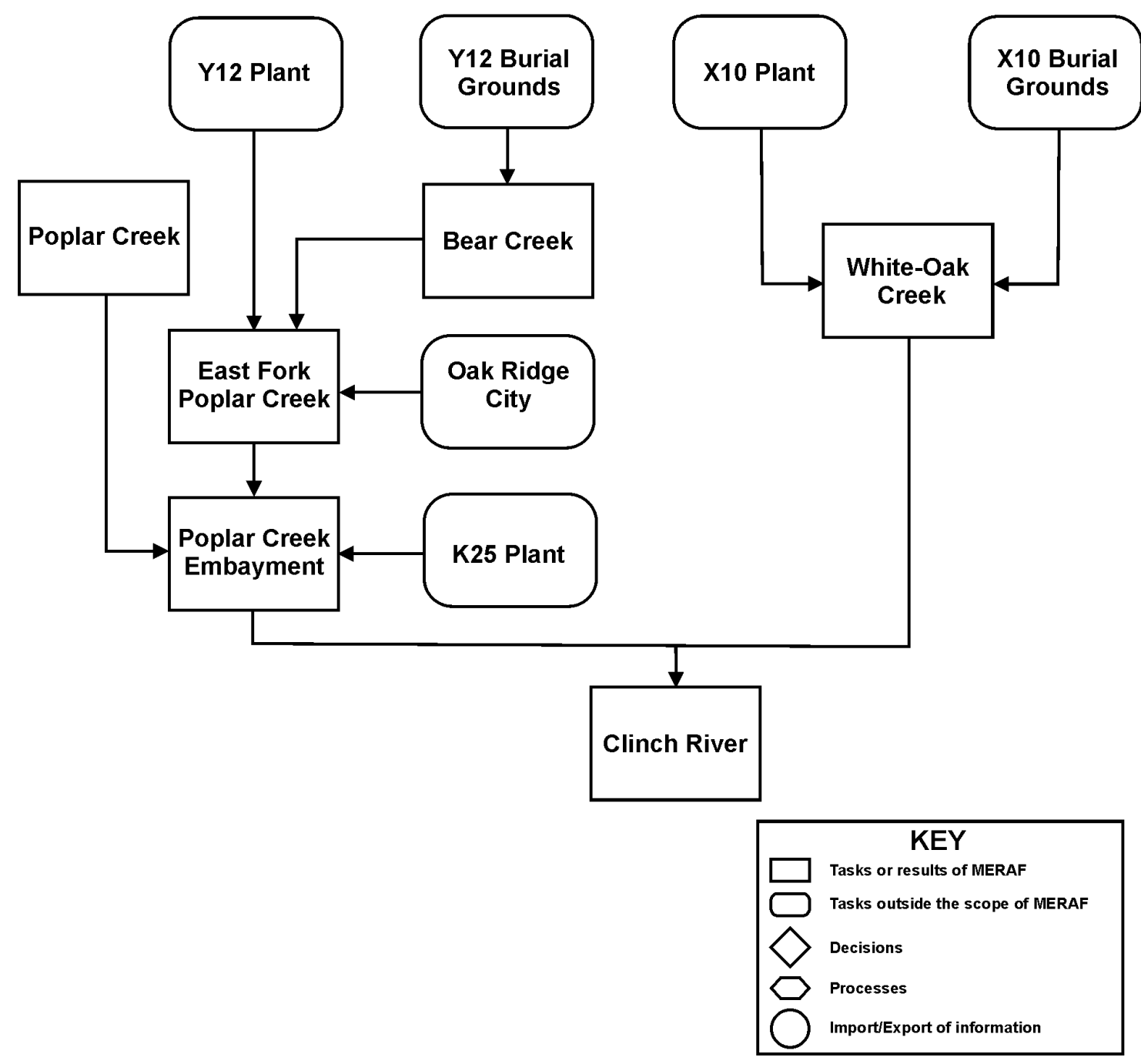

Figure 3.6-6. Example of a site conceptual model, the hydrology of the Oak Ridge Reservation.

Conceptual models of this sort, termed influence models, have been an important tool in ecology. Andrewartha and Birch (1984) provide an excellent discussion of these models and several examples. They recommend defining influences as resources, predators, malentities, and mates. Resources include food, water, nutrients, and habitat features, such as nest sites. Predators include hunters, pathogens, parasites, and conventional predators. Malentities include toxicants, extreme weather, fire, and tillage. Mates include both the presence of potential mates and the actual availability for reproduction that may be influenced by weather, habitat conditions, or human disturbance. Each of these categories should be considered when developing a receptor model. However, not all need to be included. For example, Andrewartha and Birch (1984) left mates out of most of their models because the availability of mates does not limit most animal populations. A simple example is presented in Figure 3.6-7.

This is a particularly important point at which to consider the mode and mechanisms of action. That is, given a definition of the endpoint (red-cockaded woodpecker abundance), define the proximate modes of action (e.g., survival and fecundity), and the mechanisms by which they may be altered (e.g., reduced habitat area, direct lethality, behavioral disturbance). This will facilitate linkage to the activityspecific conceptual model which defines the action mechanisms of the stressors (Sec. 4.1.4g). 


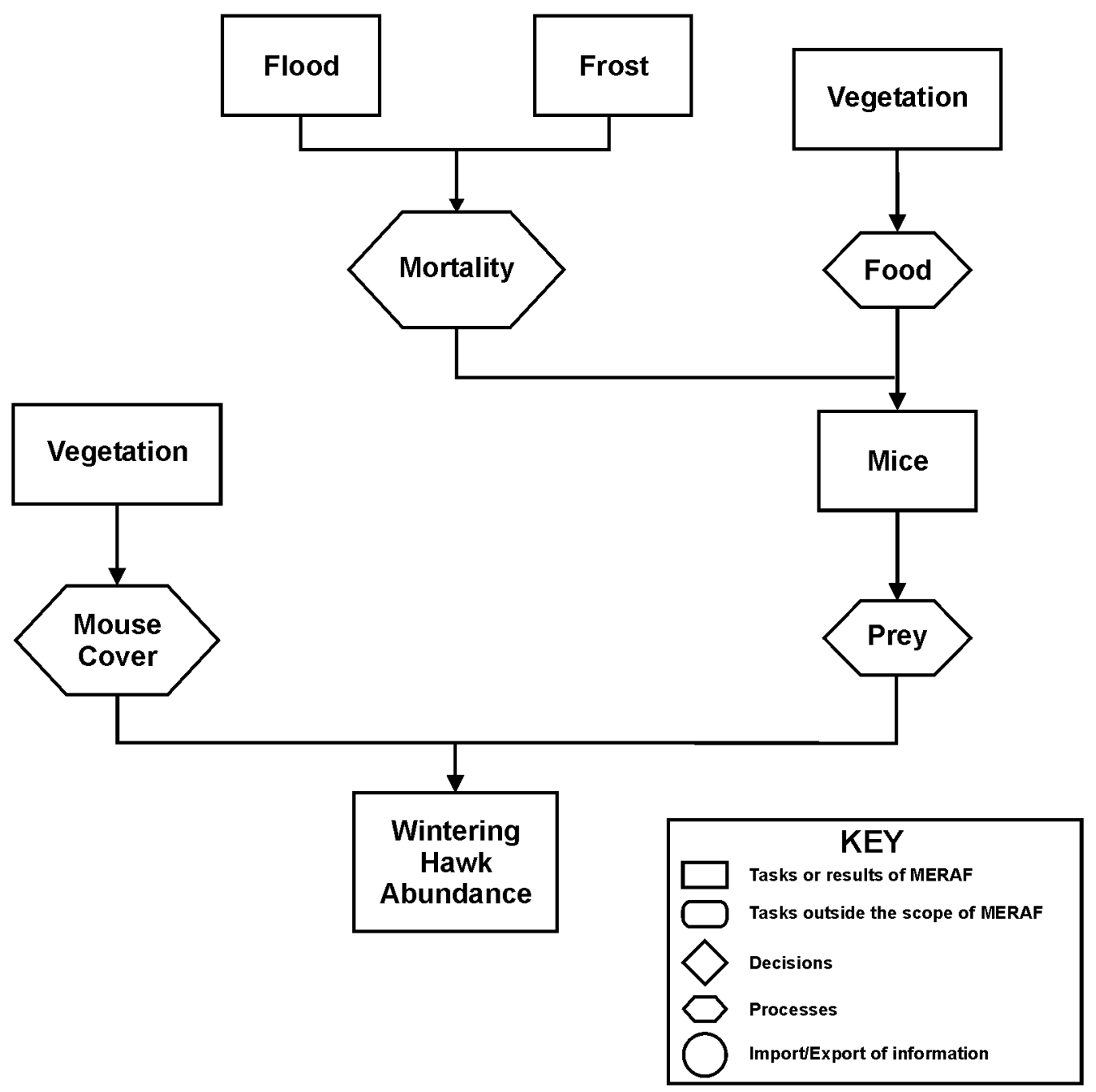

Figure 3.6-7. Example of a receptor conceptual model: wintering hawks in Michigan, based on research of Craighead and Craighead (1956) and modified from a diagram in Andrewartha and Birch (1984).

\subsubsection{Integrate the Programmatic Conceptual Model}

Ideally, this component of the problem formulation would consist of linking the site conceptual models, the receptor-based conceptual models, and the appropriate generic activity-specific conceptual models (see below and following).

\subsubsection{Assemble generic activity-specific conceptual models}

For each activity that will be performed as part of the training or testing program, obtain its generic activity-specific conceptual model, if available (Sec. 4.4g). In some cases, this may require some judgment to determine whether a proposed new activity is a specific case of a generic activity for which a conceptual model is available. In some cases, it will be necessary to modify existing conceptual models to incorporate changes in the way the activity is conducted, changes in the materials or equipment used, or site-specific and program-specific features of the activity (Sec. 4.4i). 


\subsubsection{Generate activity-specific conceptual models for other activities}

For those activities that are part of the program and do not have a generic conceptual model, develop a conceptual model that links the specific stressors associated with the activity to the assessment endpoints. Methods are described in $4.5 \mathrm{~g}$.

\subsubsection{Link to site-specific conceptual models}

The activity-specific conceptual models portray cause-effect linkages generically. To make them applicable to the programmatic assessment, they must be integrated with knowledge of the site.

\subsection{Link to site-specific hydrologic conceptual models}

The hydrologic conceptual model for the site should be obtained or generated. The following should be linked to this model:

- Any consequences of specific activities that could cause contaminants to enter aquatic systems directly or indirectly

- Any consequences of specific activities that could cause soil erosion

- Any consequences of specific activities that could cause significant changes in the hydrologic regime

- Any branches of the hydrologic model for the site that would not be altered by any of the specific activities should be deleted.

\subsection{Link to site-specific food web conceptual models}

The following should be linked to the food web conceptual model for the site:

- Any consequences of specific activities that could cause contaminants to enter food webs

- Any consequences of specific activities that could cause significant secondary effects due to loss or alteration of trophic resources of endpoint species

- Any branches of the food web conceptual model that are not involved in contaminant transfer or secondary effects should be deleted.

\subsubsection{Determine spatial co-occurrence with relevant communities}

Some consequences of activities depend on their occurrence in certain types of biotic communities. For example, disturbance of red-cockaded woodpeckers occurs only in the vicinity of mature pine stands.

- Any consequence that occurs in a relevant community should be retained in the conceptual model

- Any consequence that occurs in multiple communities to different degrees or in qualitatively different manners should be represented as a separate causal sequence for each community

- Any potential consequence that would not occur in a relevant community should be deleted.

\subsubsection{Determine temporal co-occurrence with endpoint receptors or intermediate entities}

Some consequences of activities depend on their occurrence at certain times of the year. For example, churning of soils by vehicle or foot traffic occurs only during periods when the soils are saturated or nearly saturated. Similarly, migratory species are not directly exposed to training or testing activities when they are absent from the military site.

- Any consequence that occurs in a relevant time period should be retained in the conceptual model

- Any consequence that occurs multiple times to different degrees during given time periods (e.g., different seasons or day and night) or in qualitatively different manners should be represented as a separate causal sequence for each time

- Any potential consequence that would not occur at relevant times should be deleted. 


\subsubsection{Link to receptor conceptual models}

Obtain any receptor conceptual models that are relevant to the site. Determine which direct or indirect effects of the activities, occurring in the linked activity-specific and site-specific conceptual models, are changes in the states of environmental characteristics that appear in the receptor's conceptual models and make the appropriate linkages.

\subsubsection{Link other endpoint receptors}

Endpoint entities that do not have existing receptor conceptual models should be linked based on their habitat requirements, food habits, and other environmental features that influence their endpoint properties.

\subsubsection{Review the Model for Completeness and Reasonableness}

For each combination of stressor and endpoint receptor, consider whether 1) all significant causal linkages are included and 2) all portrayed linkages are potentially significant.

Examine cause-effect linkages for redundancies and eliminate those that are detected. In particular, look for stressors that have common modes of activity (e.g., soil compaction), and combine their consequences in the conceptual model.

\section{6r CONCEPTUAL MODELS: RESOURCE MANAGEMENT}

Conceptual models for assessments of risks resulting from resource management activities are assembled from the same components as those for training and testing programs and for risks to one resource due to management of another. However, the conceptual priority is the opposite of that for programmatic assessments and, therefore, the order of assembly is reversed. That is, one begins with a receptor conceptual model for the managed resource and links it to the activities that may affect it through site-specific conceptual models. A second difference is in the pruning of unconnected branches. Influences on the resource that are not connected to a training or testing activity (e.g., poaching) should be retained if they are likely to significantly affect the resource at the facility.

\subsection{ASSESSMENT PLAN}

The assessment plan explains how the necessary components will be developed, assembled, and analyzed to complete a risk assessment that produces useful and credible results. It includes the following tasks:

Define data needs. Data needed to perform the assessment and their sources must be defined. If data must be generated by sampling, analysis, or measurement, the plan for obtaining those data must be developed and presented. In particular, it is necessary to define, for each combination of activity and assessment endpoint, what measures of exposure and effects will be used (Sec. 4.5 and 4.6).

Define model needs. Mathematical and statistical models needed to perform the assessment and their sources must be defined. If models must be generated ad hoc, the types of models and the plan for generating them must be defined and presented.

Define the organization. In complex assessments with multiple activities, multiple endpoints, and directly and indirectly induced effects, it is easy for the assessors to get lost and easier for the reader to get lost unless the assessment is clearly organized. In general, it is appropriate to complete the entire assessment for each endpoint. That is, after completing the programmatic problem formulation, assess the risks from all activities to the fish community, to bald eagles, etc. This is preferable to completing the assessment of all endpoints for activity $a$, then activity $b$, etc., or doing the exposure assessments, then the 
effects assessments, then the risk characterizations. The primary exception is when the risks result from a causal chain in which some of the intermediate effects are also endpoint effects. In such cases, it is likely to be clear and efficient to follow the entire causal chain.

Define the output. It is important to specify the form the results will take, including expression of uncertainty.

Define the schedule and effort levels. It is necessary to define the time available and needed to complete the assessment tasks and to assure sufficient money is available to fund personnel with the requisite expertise.

Plan, measurement, sampling, and analysis. Although many training and testing assessments must be completed using existing data, any ad hoc measurement, sampling, and analysis must be defined in the assessment plan. The plan should be sufficiently detailed to ensure the data collected will meet the needs of the assessment.

Quality assurance (QA) and quality control (QC). The assessment must be assured of having sufficient quality for the decision. This includes not only the conventional QA/QC for sampling and analysis, but also QA for the assessment itself. Assessment QA includes verification of mathematical models and their codes by checking results against hand calculations, and verifying that input data sets contain the appropriate data.

Summarize results of the problem formulation for review. Completion of the assessment plan is the last point at which decisionmakers and stakeholders should try to modify or redirect the process. After this point, to avoid potential biases, the scientific and technical processes of assessment should continue without policy review until the risks have been estimated. Therefore, the problem formulation should be summarized so that the basis for the assessment plan is clear and all parties agree with its logic. 


\section{ACTIVITY-SPECIFIC PROBLEM FORMULATION}

The activity-specific problem formulation defines the activity, assessment endpoints, spatial and temporal scales, the conceptual model, and appropriate measures of exposure and effects. The process is represented in Chart 4. Note that the text in the activity-specific sections (Sec. 4-8) primarily deals with the components of generic activity-specific frameworks, with only brief discussions of implementing the generic frameworks in a programmatic assessment. That is because this document is intended to guide the development of the generic frameworks, which should provide the specific guidance for implementation.

\subsection{CHARACTERIZATION OF THE ACTIVITY: GENERIC}

The generic characterization of the activity describes those characteristics and components of the activity that will be found in all, or nearly all, instances. In effect, it is a description of the characteristics of a generic activity that are relevant to performing an ecological risk assessment. Most importantly, it describes the stressors generated by the activity and their modes of action. In many cases, there will be a single significant stressor that has a single mode of action. When testing torpedoes, for example, the stressor is the explosion and the mode of action is concussion. However, some activities may be complex. For example, tank training generates noise, crushes plants, compacts soil, etc. Some judgment is required to determine what constitutes a distinct activity.

\subsection{1g Candidate Activities}

Activities associated with DoD training and testing, including resource management activities if appropriate, should be identified based on their relevance to the problems and issues of concern. Since the development of activity frameworks may require a fair degree of effort, the manager may wish to limit the number of activities to be studied, choosing only those with highest impact and sensitivity.

\subsection{2g List Component Actions}

This step involves listing the individual actions that are components of the activity that generate risks to natural and cultural resources. In training bomber crews, for example, flying the plane over the range and dropping the bombs are relevant components because they generate noise and impact. However, reviewing the flight list and navigating to the target are not relevant.

\subsection{3g Is the Action a Consistent Component?}

In general, an activity should consist of those actions that will always, or nearly always, be performed together. For example, tank training may or may not include firing live ammunition, so tank maneuvers and firing tank cannons would be separate activities within a tank training program.

\subsection{4g Define the Stressors and Modes of Action}

For each component action, it is necessary to define what is generated or released by the action that could constitute a stressor to a natural resource. Then for each stressor, the modes of action of the stressor should be as specific as possible. For example, the stressor generated by testing a torpedo is the explosion and the mode of action is concussion. For infantry maneuvers, one stressor is the presence of personnel and a mode of action is generation of flight responses.

\section{1i CHARACTERIZATION OF THE ACTIVITY: IMPLEMENTATION}

The implementation characterization of an activity describes those characteristics and components that are specific to the program being assessed. For example, if the activity is testing of 
artillery rounds, then the number and type of rounds to be tested, the dates and times of the testing, the locations of the testing, and other pertinent information must be provided.

\section{2g ACTIVITY-SPECIFIC ENDPOINT SELECTION: GENERIC}

The input to the programmatic endpoint selection process (Sec. 3.5) from the generic activityspecific problem formulation is a list of criteria to define susceptibility to the associated stressors. These criteria should be as specific as possible, but they obviously cannot address individual species and ecosystems. An example of such a criterion would be: wetland plants are susceptible to passage of vehicles. Susceptibility depends on both the potential magnitude of exposure and the inherent sensitivity of the receptor.

\subsection{1g Properties That Increase Exposure}

Organisms and other receptors that are likely to be highly exposed are more susceptible. Although exposure depends on the nature of the stressor and its release or use, some general principles can be applied when identifying susceptible entities. They include the following:

- More upright organisms are more likely to intercept shrapnel, bullets, and energy from explosions.

- Less mobile organisms are more susceptible to stressors that might be detected and avoided.

- Mature trees are less likely than herbaceous vegetation and small trees to be crushed by vehicles.

- Fully aquatic organisms are more exposed than terrestrial or semiaquatic organisms to aqueous contaminants.

- Earthworms are more exposed to soil contaminants than are soil arthropods or terrestrial animals that do not inhabit the soil.

- More active organisms have greater exposures to contaminants.

\subsection{2g Properties That Increase Sensitivity}

Although sensitivity depends on the nature of the stressor, some general principles can be applied when selecting entities. They include the following:

- Species closely related to sensitive species are also likely to be sensitive. The sensitivity of species on the site to contaminants is most likely to be predicted by the sensitivity of the most closely related, tested species (Suter et al. 1983; Fletcher et al. 1990; Suter 1993).

- There are no consistently most sensitive species or taxa, but daphnids are, on average, more sensitive to contaminants than other aquatic species (Host et al. 1991).

- Properties of higher organizational levels are less sensitive than properties of their components. Living systems cannot be more sensitive than their most sensitive component, and compensatory mechanisms typically make them less sensitive (O'Neill et al. 1986; Suter 1995a,b). For example, healing compensates for loss of cells, and increased food supplies and decreased crowding allow surviving members to compensate for losses by improved survival and reproduction.

- Long-lived species with low fecundities are especially susceptible to population declines due to adult mortality.

- Species with long maturation periods are slow to recover.

- Perennial plants with bulbs, rhizomes, or corms are less sensitive to the destruction of their leaves and stems.

- Less cohesive soils, on greater slopes, with less cover, are more susceptible to erosion.

\subsection{3g Classes of Potential Endpoint Entities}

This task is equivalent to the identification of candidate assessment endpoints (Sec. 3.5.1), except there is no particular site with real species and communities. Rather, the list for generic framework 
development must be composed of broad categories of species and communities likely to respond in a similar manner to species that have been important on military training and testing facilities.

\section{Species Categories}

- Ungulate mammals

- Small herbivorous mammals

- Small omnivorous mammals

- Ground invertebrate-feeding mammals

- Bats

- Predatory mammals

- Piscivorous mammals

- Aquatic herbivorous birds

- Arboreal insectivorous birds

- Flying insectivorous birds

- Ground invertebrate-feeding birds

- Predatory birds

- Piscivorous birds

- Herbivorous reptiles

- Insectivorous reptiles

- Predatory reptiles

- Salamanders and newts

- Frogs

- Toads

- Fishes

- Benthic invertebrates

- Soil invertebrates

Community/Ecosystem Classes

- Wetlands

- Riparian communities

- Forests

- Grasslands

- Shrub and Shrub-steppe

Species Important to DoD

- Red-cockaded woodpecker

- Desert tortoise

- Marine mammals

- Others.

\subsection{4g Criteria for Susceptibility}

Criteria for susceptibility to an activity are input to the programmatic endpoint selection process (Sec. 3.5). Criteria for susceptibility may be defined in terms of characteristics (e.g., inability to avoid oncoming vehicles), membership in a susceptible class (e.g., cetaceans for marine explosions), or a combination (i.e., members of a class with particular characteristics).

\subsection{5g Generic Activity-Specific Endpoints}

The development of a generic activity-specific framework requires identifying a limited number of generic endpoint entities and properties. These should be selected from the categories listed in Sec. 
4.2.3g, but modified as appropriate to capture the characteristics that make an endpoint susceptible to the activity (Sec. 4.2.1g and 4.2.2g). For example, if an activity may contaminate sediments with a persistent and bioaccumulative chemical, then flying insectivorous birds and mammals that forage over water would be appropriate endpoint entities.

\section{2i ACTIVITY-SPECIFIC ENDPOINT SELECTION: IMPLEMENTATION}

When implementing MERAF, this component consists of determining which programmatic assessment endpoints (Sec. 3.5) are relevant to this activity. That is, the assessment of an individual activity should include endpoints that are potentially significantly affected by the activity.

\section{3g DEFINITION OF SPATIAL AND TEMPORAL EXTENT: GENERIC}

In general, the spatial and temporal scales for an activity can be established as a core area or time and an influence area or time (Sec. 3.4). The core area and time for a training or testing program are the area and time in which the activities of the program are carried out. The area and time of influence are the areas that are affected by activities in the core area and the time over which effects occur after the activity ceases. For example, activities that increase erosion will affect streams and other aquatic systems downstream of the areas that are eroded, and those effects will continue to occur due to accumulation of sediment after the training or testing program ends. These extensions of the area of influence depend on the transport of contaminants (e.g., smokes), other materials (e.g., silt), and energy (e.g., concussion and noise). The area and time of influence are functions of the conceptual model (Sec. 4.4). That is, they depend on the assumptions about what routes of transport and what secondary effects are important to include.

The area affected is usually a function of time. That is, some areas will be affected only while the activity is being carried out, while others will be affected for some additional time by residual exposure or effects. In addition, residual exposures and effects diminish over time. If the intensity of residual exposure or effects varies over space, the area of influence will diminish over time. To the extent that they are relevant to the goals of the assessment, these relationships between area influenced and time should be defined.

\section{3r SPATIAL AND TEMPORAL BOUNDS: RESOURCE MANAGEMENT}

For assessing risks from resource management activities, the spatial scale is determined by the range of the species, the extent of the ecosystem, or the area occupied by the cultural resource, and by the core and influence areas in which activities might affect the resource. This area may extend off the facility if the population, ecosystem, or cultural resource being managed extends off the facility. No matter how the managed resource is defined operationally, it need not be completely isolated within the area assessed. For example, populations of animals on a facility receive immigrants from other populations off the facility, but those populations may be outside the scope of the management plan and, hence, of the assessment.

The core area for a resource is the area in which a management activity will be carried out. The area of influence is the area in which activities may indirectly affect the resource. There are no core and influence times for resources, however, since the management plans cover all seasons and stages in the life history of the resource. Since resources are managed for persistence in perpetuity, the time scale is simply the time horizon of the management plan. 


\section{3i SPATIAL AND TEMPORAL BOUNDS: IMPLEMENTATION}

When implementing an activity-specific framework, it is necessary first to define the core area and time period as specified in the assessment plan (Sec. 3.4). That is, define an actual area on the ground, in air space, or at sea. Then, using information concerning potential transport processes, indirect effects, site-specific transport processes (Sec. 3.6.1), and ecological relationships of the receptors (Sec. 3.6.2), define the influence area. Finally, using information concerning the degradation or loss rate of the stressors, and recovery rate of the system, define the influence time.

\section{4g ACTIVITY-SPECIFIC CONCEPTUAL MODEL: GENERIC}

The generic conceptual model for a specific activity describes the causal relationship between an activity and effects on categories of susceptible receptors. It is intended to provide a basis, along with site conceptual models and receptor conceptual models, for the development of programmatic conceptual models (Sec. 3.6). Therefore, the model should follow the standard structure of alternating processes and states (Fig. 3.6-2 and 3.6-3). Because it is generic, it should include all potentially important routes and induction mechanisms for effects that would occur at appropriate training or testing sites. Similarly, it should include all classes of receptors that could be significantly affected at any site. However, it should not define individual species or communities, except when they are known a priori to be important to the activity.

A hypothetical example of a generic model for tank maneuvers is presented in Fig. 4.4-1. This simple example was created to illustrate the discussions that follow. The narrative accompanying the model should address the following points in deriving the model.

\subsection{1g Determine the Nature of the Stressor}

The stressors associated with an activity are defined in Sec. 4.1. For conceptual model development, stressors should be divided into those that are releases of a contaminant chemical or material, those that are introduced organisms, and those that act on receptors through a physical mechanism. Conceptual models for contaminants are different from other stressors in that the fate and transport processes must be considered. Conceptual models for introduced organisms are also different in that the reproduction and activity of the organisms must be considered.

\subsection{2g Define the Mode of Propagation of Physical Effects}

Although some physical effects are generated by direct contact (e.g., tanks crushing plants), others result from propagation of energy or materials (e.g., propagation of energy from explosions; propagation of fires to combustible materials; radiation; and transmission of noise through air, water, or soil). These modes should be identified in the conceptual model along with factors that limit propagation.

\subsection{3g Define the Mechanisms of Direct Physical Effects}

Mechanisms of direct physical effects are those that cause effects when the receptor directly contacts or co-occurs with a physical stressor. They include direct contact (e.g., pressure on soil and associated organisms, as in tank maneuvers), direct exposure to released energy (e.g., concussion from explosions and burning from heat), and visual or auditory signals (e.g., alarm from sight or noise). To the extent possible, these mechanisms are specified in the characterization of the activity (Sec. $4.1 .4 \mathrm{~g}$ ). However, the process of generating a conceptual model often leads to a more complete description of the mechanisms by which physical effects are induced. 

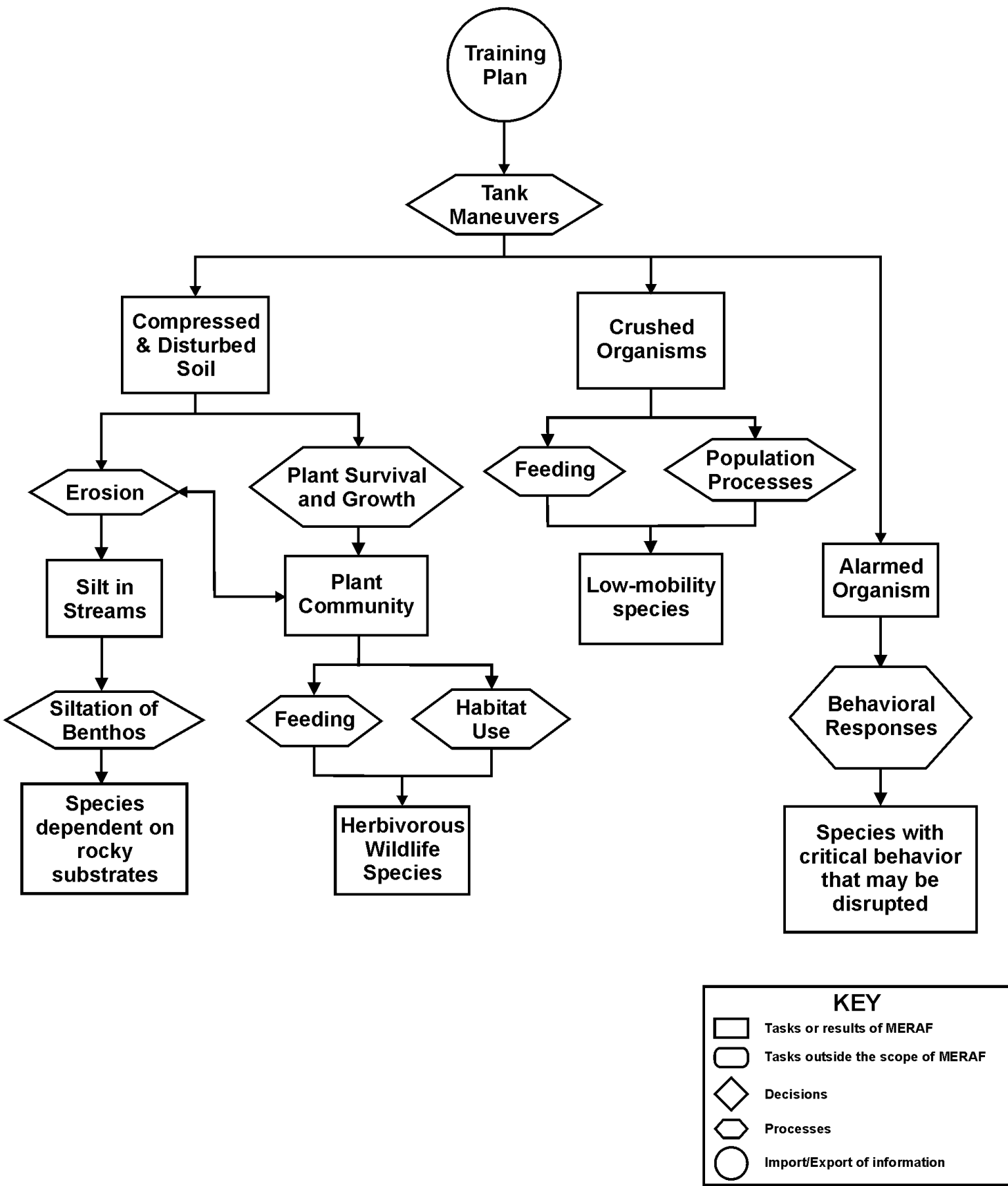

Figure 4.4-1. A hypothetical generic conceptual model for the activity "tank maneuvers." 


\subsection{4g Define the Mode of Reproduction and Spread of Introduced Organisms}

The conceptual model for an introduced organism must begin by considering how the organism reproduces and spreads. These processes determine the likelihood of a species arrival not yet on site, the rate and pattern of spread of an organism recently arrived or deliberately introduced, and the rate of reintroduction following control or eradication measures.

\subsection{5g Define the Factors Limiting Introduced Organisms}

Limiting factors determine what areas or waters may be inhabited by an organism and the rates of growth, reproduction, and other biological activities. Examples include soil moisture, water hardness, presence of prey or host species, and concentrations of nutrient elements. Those that are important to the conceptual model are likely to limit the organisms' effects on the endpoints (e.g., ability to control the pest species in the case of an introduced biocontrol agent).

\subsection{6g Define the Activity of Introduced Organisms}

The activity of the organism includes everything it does that might significantly alter the environment. Examples include consumption of weeds, pests, or native species; burrowing, overgrowing native species, competition with native species, and disease induction in weeds, pests, or native species. To the extent possible, these activities are specified as mechanisms of action in the characterization of the activity (Sec. $4.1 .4 \mathrm{~g}$ ). However, the process of generating a conceptual model often leads to a more complete description of the mechanisms by which effects are induced, so the issue must be reconsidered.

\subsection{7g Describe the Transport, Deposition, and Transformation of Materials}

This section describes the processes of transport, deposition, and transformation of chemicals and materials (e.g., fuels and smokes). It must take into consideration the properties of the chemical (e.g., water solubility) and properties of the environment (e.g., precipitation rates) that determine the relative rates of these processes. If the material is likely to be transformed into materials that could accumulate to significant levels, those products should be noted, and, if their fate or effects are different from that of the parent material, it should be added to the conceptual model. This includes not only partial breakdown products of organic materials, but also transformations of inorganic materials (e.g., formation of organomercury and organoselenium compounds from inorganic forms and oxidation and reduction of metal ions).

\subsection{8g List the Contaminated Media}

All media that would be directly contaminated by the released material should be noted, as well as media that are likely to be significantly contaminated by transported material.

\subsection{9g Define the Routes of Exposure}

Routes of exposure are the means by which a chemical or material is taken up or contacted by organisms. They may include the following:

- Ventilation of gills

- Ventilation of lungs

- Dermal (or equivalent) contact

- Ingestion of food

- Ingestion of water

- Ingestion of soil or sediment

- Grooming and preening 
- Root uptake

- Stomatal and cuticular uptake

- Atmospheric deposition

- All potentially significant routes of exposure should be included in the conceptual model.

\subsubsection{0g Define the Mechanisms of Indirect Effects}

Mechanisms of indirect effects result from the changed environmental state following direct effects of physical, biological, or chemical stressors. Examples include loss of habitat structure and loss of trophic resources (e.g., food plants in Fig. 4.4-1).

\subsubsection{1g More Stressors?}

The example (Fig. 4.4-1) illustrates an activity (tank maneuvers) that generates two stressors: 1) pressure of tank tracks on plants and animals they pass over, and 2) noise. If more than one stressor is associated with an activity, the process of generating the conceptual model must go down the branches of Chart 4.4g more than once. For example, completion of the conceptual model required completing 4.4.2, 4.4.3, and 4.4.10 for noise, as well as for pressure.

\subsubsection{2g Generate the Model}

The generation of the generic conceptual model for an activity requires linking the activity to the endpoint entities through the processes and resulting states defined in Sec. 4.4.2 through 4.4.10. The potential endpoint entities can be drawn from the process of generic endpoint selection (Sec. 4.2.5).

\section{4i ACTIVITY-SPECIFIC CONCEPTUAL MODEL: IMPLEMENTATION}

Implementation of an activity-specific conceptual model consists of performing the necessary steps of the programmatic conceptual model (Sec. 3.6). They include:

- ensuring that the planned activity will generate all the stressors indicated in the model and no more,

- determining whether changes in equipment, materials, or practices require changes in the routes and mechanisms,

- deleting routes and mechanisms irrelevant to the site, and

- linking the model to other activity, site, and receptor models.

An implementation of the hypothetical generic conceptual model for tank maneuvers shown in Fig. 4.4-1 is based on the conceptual model in Fig. 3.6-2. The simplification in this case results from implementation of the model for a desert site and the selection of desert tortoise abundance and plant diversity as assessment endpoints. For those endpoints, alarm due to noise is not a significant issue. The lack of streams in this site conceptual model eliminates siltation as a mode of action. The addition of exposure-response relationships to the conceptual model during implementation as shown in Fig. 3.6-2 clarifies the relationship of the model to the assessment process.

\section{5g SELECTION OF ACTIVITY-SPECIFIC MEASURES OF EXPOSURE: GENERIC}

This section defines how exposure will be quantified for activity assessments. Measures of exposure are the metrics used to define the degree of exposure of endpoint receptors to the stressors. These measures must be specific to the stressor and the receptor. For chemicals, measures of exposure are typically concentrations in an environmental medium (e.g., surface water or soil). Measures for physical stressors are much more diverse. For example, for explosions the measure may be maximum pressure, but for passage of vehicles it may be the number of vehicles traversing a particular plot of land. 
The measures of exposure must be identified during the problem formulation so that:

- they will correspond to the metric used in the exposure-response relationship (Sec. 4.6),

- necessary information concerning the activity is obtained from individuals planning the training, testing, or resource management program (Sec. 4.1), and

- necessary measurements or other data will be collected.

If the conceptual model indicates that risks result from indirect effects, then measures of exposure must be identified for each intermediate exposure, as well as the exposure of the final endpoint entity.

It is not sufficient to specify the measure of exposure in a general way. Rather, the following dimensions of exposure should be defined for each measure.

\subsection{1g Define Intensity Measures}

Intensity is the measure of magnitude of the stressor that renders it capable of inducing an effect. That is, it is the concentration, pressure, or other characteristic that determines how strongly the stressor acts on the receptor. It depends on the mode of action of the stressor.

\subsection{2g Define Temporal Measures}

The temporal dimensions of the measures may include duration, frequency, timing, or combinations of those dimensions. Temporal dimensions are discussed in more detail in Sec. 5.5.2.

The duration of exposure determines the magnitude of response to a particular intensity of a stressor. In the simple case of an activity that leaves no residue, the duration is the length of time the activity is performed. Examples include the duration of a noise or the passage of a pressure wave. In other cases, duration of exposure includes both the duration of the activity and the continued occurrence of significant residues of that activity in the environment.

The frequency of occurrence of a stressor at a particular location determines individual instances of exposure. If enough time elapses between instances, then recovery will occur and exposures are independent. If the time between instances is small relative to the rate of recovery, then exposures are fully additive. If the time between instances is intermediate, then exposures are partially additive.

The timing of the exposure is the actual time interval during which the exposure occurs. These may be simple dates and times, but it is often important to define the timing of exposure relative to seasonal events. Examples include the thawing of the soil, the presence of migratory species, or diurnal events, such as sunrise and sunset, or tides.

\subsection{3g Define Spatial Measures}

Spatial measures specify the area over which exposure occurs and any relevant shape considerations. For example, exposure may occur in corridors or be distributed over a broad area. The spatial extent of exposure is likely to be quite variable among sites, but the metrics should be common across sites and programs.

\section{5i SELECTION OF ACTIVITY-SPECIFIC MEASURES OF EXPOSURE: IMPLEMENTATION}

When implementing a risk assessment, the generic measures of exposure must be reconsidered to confirm their applicability to the specific case being assessed. If necessary, more appropriate measures should be selected and defined in terms of the dimensions discussed above (Sec. $4.5 \mathrm{~g}$ ). Whether generic or program-specific measures are used, they must be defined in terms of what existing data will be used, what additional information is needed from the program planner, what new measurements will be taken, and what models will be run. 


\section{6g SELECTION OF ACTIVITY-SPECIFIC MEASURES OF EFFECTS}

Measures of effects are the metrics used to define the effects of exposure. For toxicological effects, measures are either exposure-response relationships or benchmark exposure levels that correspond to some threshold effect. For physical agents, equivalent metrics must be selected, such as sound levels that result in auditory injury or relationships between the number of passages of a vehicle and plant mortality. Measures of effects may be derived from laboratory studies (e.g., toxicity tests with smokes), or field studies onsite or elsewhere (e.g., studies of the response and recovery of plant communities on artillery ranges). If the conceptual model indicates that risks result from indirect effects, each intermediate effect must be identified, as well as the final endpoint effect. The generic framework should indicate what measures are appropriate for assessing the activity.

\section{6i SELECTION OF ACTIVITY-SPECIFIC MEASURES OF EFFECTS: IMPLEMENTATION}

When implementing the activity-specific framework, one would choose measures of effect that are most appropriate to the site, the specifics of the activity, and the conceptual model. 


\section{5g. CHARACTERIZATION OF EXPOSURE}

Resources must be exposed to a stressor for an effect to occur. Characterization of exposure is the estimation of expected intensity, time, and extent of co-occurrence or contact with a chemical or other stressor by receptors. Along with characterization of effects, it constitutes the analysis phase of the assessment. In risk assessments, the analysis of exposure provides an appropriate exposure estimate to paramaterize the exposure-response model (Sec. 6) so that risk can be characterized (Sec. 8). The concordance between measures of exposure and effects should have been ensured during their selection (Sec. 4.5 and 4.6). The activities described in this section (Chart 5g) quantify the exposure measures using the problem formulation results, the measurement results, and, if the effects are indirectly induced, the intermediate risk characterization results (Sec. 7).

\section{1g CHOOSE THE EXPOSURE ESTIMATION METHOD}

For each endpoint and stressor, it is necessary to determine the most appropriate method for estimating the exposure. If the characterization of the activity provides a sufficient description of the exposure, then additional analysis is unnecessary. If exposure is not only a function of the activity, but also depends on environmental characteristics, then empirical exposure models are likely to be appropriate. Based on studies of the same activity occurring on the same site or similar sites, empirical models may be developed or may already exist. If development of empirical models is not practical or if the stressor is novel, mathematical exposure models are needed. They are also needed if the exposure is too complex to model empirically because of multiple mechanisms, routes of exposure, or multiple environmental factors that vary independently.

The principal input to the generic characterization of exposure is the results of the problem formulation (Chart 4), which specify the activity, conceptual model, endpoints, and measures of exposure. In addition, if the exposures are indirect, then the intermediate risk characterization replaces the activity description as the source of stressors (Chart $7 \mathrm{~g}$ ). Finally, the characterization of exposure must be performed along with the characterization of effects (Chart 6g) so that the exposure metrics and effects metrics can be combined to estimate risks.

\section{2g DESCRIPTION OF THE ACTIVITY}

In many cases, the characterization of the activity (Sec. 4.1) will provide a sufficient quantitative description of the exposure. For example, if the concern is that the presence of personnel will frighten individuals of an endangered species causing them to abandon habitat or modify their behavior, no model is needed to estimate that exposure. The number of individuals, the time that they will spend in the area, the number of visits, and the intervals between visits constitute a complete description of the exposure.

\section{3g EMPIRICAL MODELS OF EXPOSURE}

Empirical models are a generally useful, but underutilized, approach to exposure estimation. Many training and testing activities are repetitive. Therefore, it is often feasible to take measurements of exposure following activities, and use them to develop models of the intensity, duration, frequency, or area of exposure as a function of the intensity, duration, frequency, or area of the stressor. An example is the development of soil compaction models as a function of tracked vehicle traffic. In many cases, empirical models must include environmental variates if they are to have acceptable precision. For example, soil compaction is a function of soil texture as well as tracked vehicle traffic.

\section{4g MECHANISTIC MODELS OF EXPOSURE}

If there is a mechanistic understanding of the exposure process, and if it is sufficiently complex or variable that it cannot be simply described or empirically modeled, then the use of mathematical models 
is appropriate. The most common example in ecological risk assessment is the modeling of wildlife exposures to contaminants from consuming various food items, water, and soil. Another important example is noise attenuation. The physics of sound attenuation is sufficiently well known to make mechanistic modeling practical. Although a model with empirically derived site-specific absorption could be more accurate, the gain in accuracy is unlikely to be cost-effective, given the greater uncertainties in other components of the assessment.

\section{5g EXPOSURE PROFILE}

The exposure profile is the product of the characterization of exposure. It should describe the exposure of an endpoint receptor to each stressor in terms of the appropriate dimensions. Note that these dimensions often are not independent. For example, the intensity of exposure may decline over time (e.g., due to degradation of a contaminant) or over space (e.g., decline in sound over distance). Therefore, the exposure profile often must be a function. For example, the exposure to noise is a function of the energy at the source and the inverse square of the distance from the source. Variability in exposure must be identified both in terms of controlled variability among program plans (e.g., number and duration of smoke releases) and uncontrolled variability (e.g., wind speed and direction). Uncertainties in the exposure characterization must be noted, quantified as much as possible, and compiled for inclusion in the risk characterization. Note that the exposure profile should contain quantitative realizations of the measures of exposure (Sec. 4.5) generated by the chosen exposure estimation methods.

\subsection{1g Specify the Intensity of Exposure}

Intensity is the fundamental exposure variable. Whether it is concentration of a chemical, number of personnel in a bivouac, explosive force of a munition, number of tanks in a column, or the number of deer harvested in a public hunt, the intensity of exposure determines the potential for causing effects. That potential must be quantitatively defined in the appropriate units specified in the measures of exposure (Sec. 4.5.1g) and in terms of its distribution in space and time.

\subsection{2g Specify the Temporal Dimensions of Exposure}

The temporal dimension of exposure may be variously expressed as duration, frequency, timing, or some combination of those three. The appropriate dimensions should have been specified when defining the measures of exposure (Sec. $4.5 .2 \mathrm{~g}$ ).

\subsubsection{1g Specify the Duration of Exposure}

In the simplest cases, duration of exposure is the time period between beginning and ending of the exposure. If the duration is sufficiently short (e.g., the duration of an explosion or of the passage of a jet aircraft), the duration may be ignored because the exposure is essentially instantaneous. In more complex cases, duration is a categorical variable. That is, the intensity of exposure is constant within a particular time period, but there are multiple periods with different intensities, or the exposure durations differ among distinct areas. Finally, duration may vary continuously with intensity or across space.

\subsubsection{2g Specify the Frequency of Exposure}

Frequency may be expressed in different forms, depending on the stressor and receptor.

- Number of occurrences per unit time.

- Time since the last occurrence.

- Time until the next occurrence.

- Distribution of intervals between occurrences. 
For example, if the stressor is passage of tanks over a meadow, the frequency of tank passages is largely irrelevant because there is little recovery between tanks. However, the time since the last training exercise is relevant in determining whether recovery has occurred. Also, the time until the next occurrence is relevant in terms of predicting recovery. Distributions of occurrence intervals are appropriate for random occurrences, such as impacts of bombs or shells on a particular spot within a range. If an activity is not repeated on a particular site, there is no need to specify the frequency.

\subsubsection{3g Specify the Timing of Exposure}

Timing refers to the calendrical occurrence of events. In general, the most important aspect is the season in which activities occur. For example, in winter the soil may be frozen, plants may be in resting stages, and terrestrial animals may have migrated or entered hibernation, all of which would reduce exposure to training or testing activities. Timing may also need to be defined relative to other temporal cycles, such as tides, and rising and setting of the sun. In some cases, timing relative to other human activities may be important if those activities affect the susceptibility of the environment to the activity being assessed.

\subsection{3g Specify the Area of Exposure}

Space should be defined in relevant terms (Sec. 4.5.3g). For example, dietary exposures of wildlife should be averaged over areas corresponding to foraging ranges. In addition, the actual areas involved should be specified using GIS, hand-drawn maps, or defined boundaries.

\section{6g IS THIS EXPOSURE INTERMEDIATE?}

If the conceptual model indicates that risks result from indirect effects, an exposure profile must be generated for each intermediate exposure, as well as the final exposure. Therefore, unless the risks to an endpoint are entirely due to direct exposures, the characterization of exposure must be iterated for all exposures in the causal chain. The stressors that are input to subsequent exposure characterizations are changes in the environment estimated by the intermediate risk characterization (Sec. 7), as well as direct exposures to the training or testing activity (Sec. 4.1). For example, wildlife are directly exposed to tank traffic, and indirectly exposed to physically altered habitat and loss of plant food (Fig. 3.6-2). 


\section{5i. CHARACTERIZATION OF EXPOSURE: IMPLEMENTATION}

The components of exposure characterization are the same in the implementation phase as in the development of generic frameworks (Chart $5 \mathrm{~g}$ ). The principal difference is that in implementing an activity-specific framework, actual activity and environmental information are used to generate exposure estimates. The information that is available concerning the activity and environment determines what assessment methods are appropriate and feasible. Some exposure models identified by the generic framework may be inappropriate because the training or testing site is substantially different from the site for which the model was developed, needed parameters are not available for the model, or constraints on time to acquire environmental data, etc.

Another significant difference is that the exposure characterization should include any residual exposure from prior training and testing activities. If obscurants have been used previously on the site, for example, the exposure model should include residues from those prior uses, as well as the predicted residues from the planned activity. Cumulative exposures are more difficult to define for activities that do not involve the release of contaminants. It is important to distinguish cumulative exposures from cumulative effects. If effects are accumulating over time (e.g., there is a prescribed risk that tortoises are crushed by tanks during each training exercise), one may characterize the cumulative risks by combining effects during the risk characterization (Sec. 8i and 9). 


\section{6g. CHARACTERIZATION OF EFFECTS}

This section describes how to define a model of exposure effects by a particular stressor or set of stressors associated with an activity. Such models are termed exposure-response relationships. The concept is conventional in toxicology and in standard risk assessments. Examples include the linearized multistage model used by the EPA in cancer risk assessments or the probit models fit to acute lethality tests in aquatic toxicology. The concept is no less important when estimating risks of physical disturbance of other nonchemical stressors. That is, the level of expected effect increases with increasing exposure to the stressor. In general, for almost any stressor, there is some level of exposure below which effects are nonexistent or negligible. On the other hand, even little girls picking wildflowers could pose an unacceptable risk if there were enough girls picking for enough days. Hence, for most stressors and receptors, there is a threshold exposure level below which no consequential effects occur, an increasing level of effects with increasing exposure, and a level at which the maximum effects occur (e.g., extinction or complete devegetation).

The measures of effects should have been identified in the problem formulation (Sec. 4.6). If the conceptual model indicates that risks to an endpoint are indirectly induced, an effects characterization must be completed for each intermediate effect, as well as the final endpoint effect. If more than one measure of effects is used to characterize an intermediate or endpoint effect, an exposure-response relationship should be developed for each.

\section{1g CHOOSE EFFECTS MODELS}

The availability of appropriate effects models is often the most limiting factor in ecological risk assessment, particularly for nonchemical stressors. Exposure data and models are routinely generated for individual programs and sites based on program plans and site-specific information. However, toxicity tests, field studies of disturbance effects, and other effects studies are seldom performed ad hoc.

Therefore, the choice of effects models often reduces to a decision to use whatever is available. When multiple effects models or sources of effects data are available to generate models, one must decide which are useful, defensible, and potentially applicable to training and testing sites and activities. Of those that are acceptable, one must choose a set of models that represent best assessment practice and that will complement each other in the risk characterization. Models may be selected from the following categories.

\subsection{1g Empirical Models}

The fundamental concept of effects modeling in ecological risk assessment is empirical exposureresponse modeling. This approach is derived from toxicology in which groups of organisms are exposed to a series of concentrations of a chemical and a function is statistically fit to the resulting data. Such models should be used for any toxicological risks. They are applicable to any other stressors for which effects have been related to multiple levels of exposure. Empirical exposure-response models may be generated from observational, as well as experimental, data if a gradient of exposure or a graded series of exposure categories can be identified. If multiple exposure metrics have been related to effects (e.g., concentration and duration), or if environmental characteristics have been shown to interact with exposure to determine effects (e.g., water hardness and metal concentration), then multivariate regression techniques can be used to generate the exposure-response model.

\subsection{2g Mechanistic Models}

The principal advantage of mechanistic models is flexibility. That is, if one understands the mechanisms by which effects are induced, and has appropriately represented them in a mathematical model, that model can be used to estimate effects at various sites, in various seasons, and of various forms and levels of the stressor. In contrast, empirical models are limited to situations that are similar to those 
from which they were derived. This flexibility is particularly important when the processes controlling effects induction are complex and involve multiple variables. The major limitation of mechanistic modeling is the lack of mechanistic understanding (i.e., mechanisms are often unknown or incompletely known) and of models incorporating that understanding (i.e., mechanistic models are unavailable or do not fully or accurately incorporate the relevant mechanisms). Secondary disadvantages are distrust of mathematical models, lack of expertise in modeling, and lack of transparency to decisionmakers and stakeholders.

Mechanistic models are commonly used in resource management to estimate effects of harvesting on populations. Demographic population models developed for this purpose have been adapted to estimate risks from contaminants (Barnthouse et al. 1987; Barnthouse et al. 1990).

\subsection{3g Threshold Models}

Threshold models are used if the effects are dichotomous (i.e., either they occur or they do not), or, if the effects increase continuously with exposure, then a critical level of effects has been identified. In the latter case, the threshold level of effects should be identified during the problem formulation. Thresholds may be identified from empirical studies or mechanistic models. For example, when assessing risks of fish kills due to fuel spills in streams, death of $10 \%$ of fish may constitute a kill incident, and the effects model would be concentrations below the $\mathrm{LC}_{10}$ would not cause an effect, and those above the $\mathrm{LC}_{10}$ would cause an effect. As a simpler example, it may be assumed that, if diesel fuel is spilled on land, then all herbaceous vegetation is killed. When objective methods are unavailable, thresholds may be established by expert judgment. For example, experts on red-cockaded woodpeckers might judge that troops could approach within 100 meters of a woodpecker nest site without disrupting the behavior of the birds.

\section{2g RELATIONSHIP TO EXPOSURE}

The relationship between exposure and effects is largely determined by the effects model. That is, the selection of an effects model determines what exposure parameters are used. For example, most toxicity data for fish are expressed as concentration-response functions with constant duration. If tests of various durations are available for fish exposed to the contaminant of concern, then one can select the test with the most appropriate duration. However, because duration is fixed for toxicity values taken from the literature, there is not an option of expressing response as a function of duration or frequency of exposure, unless new toxicity tests are performed as part of the assessment. On the other hand, exposure is commonly determined for individual assessments and the assessor has considerable flexibility in how exposure is characterized. Due to this constraint, in most cases, the effects characterization must specify the form of the exposure characterization results.

When one is developing effects models rather than using existing models, the exposure metrics are chosen on the basis of their relationship to effects and their variability. That is, one chooses exposure metrics that are important determinants of the magnitude of effects, given the potential range of each metric. For example, if the atmospheric concentration of smokes is highly variable due to dispersion, but the durations are relatively constant, then the exposure of organisms should be modeled as a function of concentration. In this case, duration may be neglected because the concentration will determine nearly all of the variance in effects. In some cases, the multiple exposure metrics may be required for the exposureresponse model. For example, nest abandonment resulting from human disturbance may be a function of the number of troops involved, their distance from the nest, and the duration of their presence. Although the number of troops may not be used as an exposure metric because it is effectively constant (e.g., a platoon), the distance and duration are potentially highly variable and highly influential on the responses of the birds. Therefore, the effects model would need to include distance and duration. Hence, in developing exposure-response models, one should:

(1) estimate the range of variability in each potential exposure metric, 
(2) estimate how much the response is likely to change over each of those ranges, and

(3) include those metrics that are expected to significantly contribute to the variance.

\section{3g RELATIONSHIP TO ENDPOINTS}

The exposure-response relationship must generate either the endpoint response, or, if the effect is intermediate, the appropriate intermediate response. (From here on, this section will refer to endpoint responses for the sake of brevity, but intermediate responses are equivalent.) The response metrics in measures of effects seldom correspond exactly to the assessment endpoints. First, the entity often differs. For example, one may have toxicity test data for fathead minnows and the assessment endpoint entity may be an endangered fish population or an entire fish community in a receiving stream. Similarly, one may have data on responses of Alaskan peregrine falcons to jet overflights when assessing risks to another raptor species, or to the same species in a different place where the sensitivity to noise may be different (e.g., California peregrine falcons). Second, the endpoint property often differs. Continuing the fathead minnow example, the toxicity test may report survival and growth of individuals, while the endpoint properties may be persistence of the endangered population or species richness of the fish community. Extrapolation models are used to relate the measures of effects to the assessment endpoint. These models are relatively well developed in risk assessment for chemicals (Suter 1993), but additional models need to be developed for other stressors.

In developing exposure-response models, one should:

(1) List the potentially important differences between the measures of effects and the assessment endpoint (e.g., taxonomy, life stage, community type, severity, or level of organization),

(2) Determine which, if any, of those differences are important to the results of the assessment,

(3) Determine what models or data are available to perform the extrapolations for the potentially important differences,

(4) Select or develop models that account for the important differences.

Types of extrapolation methods are discussed in the following sections.

\subsection{1g Extrapolation by Expert Judgment}

The most common extrapolation technique is expert judgment, the most common expert is the scientist performing the assessment, and the most common result is that the measure of effects is judged to adequately represent the assessment endpoint. However, expert judgment may be used to derive factors to correct for differences or to derive model forms and parameters.

\subsection{2g Extrapolation Using Empirical Factors}

The most common quantitative approach to effects extrapolation is to apply a factor that is based on an analysis of cases in which the relevant difference has been quantified. For example, we may know the acute toxicity of a chemical to the endpoint species, but not the chronic toxicity. Therefore, we may use an acute/chronic factor derived from tests of the same chemical with another species, or the mean acute/chronic factor for many species exposed to related chemicals (Calabrese and Baldwin 1994).

\subsection{3g Extrapolation Using Empirical Models}

Another relatively common extrapolation approach is to develop empirical models of the relationships that characterize the extrapolation. For example, differences in sensitivity of mammalian and avian species to toxic chemicals are largely determined by their rate of elimination of the chemicals. Because elimination rates are proportional to metabolic rates (which are functions of weight), allometric scaling models have been used to extrapolate toxicity data between species (Pokras et al. 1993; Sample et al. 1996). 


\subsection{4g Extrapolation Using Mechanistic Models}

Mechanistic models used to characterize effects (Sec. 6.1.2) may also be the mechanisms that control the differences between the measured effects and the endpoint effects. For example, if the mechanisms of loss of vegetation due to vehicle passage are modeled (e.g., plant crushing, soil compaction, and soil churning), then measures of plant loss at one site can be used to estimate plant loss at other sites. In ecological risk assessment for chemicals, mechanistic models have been used to extrapolate toxic effects from individual fish to fish populations, and from various aquatic species to lake ecosystems (Barnthouse et al. 1990; Bartell et al. 1992 ).

\section{4g EFFECTS PROFILE}

For any stressor and endpoint receptor, multiple exposure-response relationships may be identified for inclusion in the generic framework. For example, a mechanistic model and an empirical model may have been developed for the same exposure-response relationship. In addition, different empirical models may have been developed at different sites, at different times, with somewhat different versions of the stressor (e.g., different obscurants), or with different receptors or responses. Each of these models should be included in the generic effects profile so that those applicable to a particular program can be selected. For each model, a guide must be provided which should include the following points:

- Form of the model

- Parameters of the model (i.e., constants), their units, and their sources

- Site-specific or program-specific parameters required to implement the model (i.e., variables including exposure parameters) and their units

- Methods for deriving those input parameters

- Output of the model, including form and units

- Relationship of the output to potential endpoints

- Situation from which the model was derived

- Relationship of that situation to the other situations where the model may be used

- Experience in use of the model in other situations (i.e., empirical validation)

- Implicit assumptions required to use the model

- Strengths relative to alternative models

- Weaknesses relative to alternative models

- Uncertainty in output of the model (Sec. 12)

\section{5g ARE EFFECTS INTERMEDIATE?}

If the conceptual model indicates that risks result from indirect exposure and effects, an effects profile must be generated for each intermediate effect, as well as the final effect. Hence, unless the risks to an endpoint are entirely due to effects of direct exposures, the characterization of effects must be iterated until all effects in the causal chain are characterized. For example, herbivorous wildlife are directly exposed to tank traffic and indirectly exposed to loss of plant food (Fig. 3.6-2). Hence, the first iteration would develop an exposure-response relationship for plant growth, and the second would develop an exposure-response relationship for abundance of the endpoint herbivore. 


\section{6i. CHARACTERIZATION OF EFFECTS: IMPLEMENTATION}

The components of the characterization of effects are the same in the implementation phase as in the development of generic frameworks (Chart 6g). The principal differences in implementing an activity-specific framework are that actual activity and environmental information are used to parameterize existing exposure-response models or to generate new ones, if none of the existing models are suitable. The information available concerning the activity and receiving environment determines what assessment methods are appropriate and feasible. Some types of models identified by the generic framework may be inappropriate because the site is substantially different from the site for which the model was developed, because needed parameters are not available for the model, because of constraints on time to acquire data, etc. The effects profile for framework implementation contains the same points as the profile in the generic framework (Sec. 6.4), except the questions must be answered relative to the actual implementation, as follows.

- Form of the model including any site-specific changes in the form

- Parameters of the model (i.e., constants), their units, and their sources, highlighting any changes from the generic parameters

- Parameters required to implement the model (i.e., variables including exposure parameters) and their units

- Methods used to derive those input parameters

- Output of the model including form and units

- Relationship of the output to the chosen endpoints

- Situation from which the model was derived

- Relationship of that situation to the situation being assessed

- Experience in use of the model in other situations, and the degree to which it validates use of the model in this situation

- Implicit assumptions required to use the model

- Strengths relative to alternative models

- Weaknesses relative to alternative models

- Uncertainty in output of the model 


\section{7g. INTERMEDIATE RISK CHARACTERIZATION}

\section{1g INTERMEDIATE RISK ESTIMATION}

If an effect is intermediate in the conceptual model (Sec. 6.5), then it is necessary to estimate those effects that serve as exposures for effects that are downstream in the conceptual model. As discussed more fully in Sec. 8, risk estimation uses the content of the exposure profile to parameterize the exposure-response models in the effects profile. This generates an estimate of the risk of the prescribed effect. The intermediate risk characterization step is different from those for the assessment endpoints (Sec. 8 and 9) because the properties to be estimated may not be inherently important or valued (Sec. 3.5). They may simply be important to the indirectly induced response of some endpoint property. Another difference is that an intermediate risk characterization must serve as the input to the characterization of exposure for the next iteration of the analysis phase. That is, rather than basing the characterization of exposure (Sec. 5) on the characterization of the activity (Sec. 4.1), it is based on the estimate of intermediate effects from this intermediate risk characterization.

\section{2g IS THE AFFECTED ENTITY AN ENDPOINT?}

If the intermediate response is also an endpoint response, then the results of the intermediate risk characterization are passed on to the activity-specific risk characterization (Sec. 8), as well as the analysis of exposure for the indirectly induced effects (Sec. 5). For example, in an assessment of effects of tank training, the effects of tank traffic on plants may be both an endpoint and an intermediate effect for endpoint wildlife species (Fig. 3.6-2). Note that the relevant risk characterization results may be different because the valued property of the endpoint entity (e.g., diversity of plants) may be different from the property that determines the effects on other endpoints (e.g., productivity of plants). 


\section{8g. ACTIVITY-SPECIFIC RISK CHARACTERIZATION}

This section describes how to characterize the risks posed by a particular activity to an endpoint receptor. It includes integration of exposure estimates into exposure-response relationships, integration of lines of evidence if multiple lines of evidence are available, combining multiple modes of action, and estimation of uncertainty. Note that the steps described in this section must be carried out for each endpoint.

\section{1g ESTIMATION OF RISKS}

Estimation of risks requires implementing the exposure-response models using the exposure estimates. If the activity generates multiple stressors or actions, they must be screened and those that may significantly contribute to the risks to an endpoint must be combined.

\subsection{1g Implementing Exposure-Response Models}

Risk estimation involves integrating the results of exposure and effects characterizations (Sec. 5 and Sec. 6, respectively). This requires that these characterizations be performed with concordant spatial, temporal, and intensity dimensions.

\subsubsection{1g Empirical models}

In classic risk assessments of chemicals, risk estimation involves plugging the estimated dose into the empirical dose-response model and estimating the magnitude of the response/risk. Other risks should be estimated analogously except when the units of exposure are not dose and the relationship between exposure and response is not a probit model relating dose to the proportion dying. For example, plant cover in native pasture has been empirically related to number of hikers trampling over a point by the polynomial model:

$$
y=-7.5 x^{2}+74 x-83
$$

where:

$\mathrm{y}=$ relative cover

$\mathrm{x}=$ number of walking passages (Liddle 1975).

Therefore, for a site similar to that studied by Liddle, estimation of cover relative to reference can be estimated by simply plugging in the number of troops.

\subsubsection{2g Mechanistic models}

Mechanistic models (also termed "process models") need not be more complex than empirical models. They can be implemented just as simply by plugging an exposure metric into an exposureresponse equation. For example, the risks from strafing might be assumed to result from being hit by a round. Exposure to strafing would be expressed as the number of rounds per meter square and the exposure-response model would be a function of the endpoint organism density in the strafed habitat and the area of the organism's silhouette. Many mechanistic models are more complex than this, but the concept is the same. That is, the exposure characteristics are parameters of the mechanistic exposureresponse model, and the model may be implemented after each of those parameters is assigned a value based on the exposure characterization (Sec. 5.5). 


\subsubsection{3g Threshold models}

Threshold exposure-response models are the simplest to implement. If the exposure level exceeds the threshold for effects, then the prescribed effect is expected to occur. If the exposure level does not exceed this threshold, then the prescribed effect is not expected to occur.

\subsection{2g Weight of Evidence}

In most cases of predictive assessments, such as NEPA documents, only one line of evidence will be available for risk characterization. However, if the characterization of effects has identified multiple exposure-response models for the activity, or if there are multiple defensible methods to derive the exposure estimates, then risks should be estimated by each method. The generic framework must present a logical basis for weighing those risk estimates to derive a best estimate.

This situation is different from the weighing of evidence for contaminated sites and other retrospective assessments that may have logically distinct lines of evidence, such as biological surveys, biomarkers, ambient media toxicity tests, and measurements of contaminant concentrations. Those lines of evidence can be used in an ecoepidemiological analysis that works from the effect (as revealed by the state of the receptors) back to the cause (specific contaminants in ambient media) (Suter 1996b). In contrast, if the alternative lines of evidence are simply different predictive models, none are logically distinct. They simply have different sources, different levels of validation, different uncertainties, etc.

\subsubsection{1g Are estimates effectively the same?}

Ideally, multiple lines of evidence will produce results that are effectively the same. That is, if the risk estimates are dichotomous variables, then the results are the same, and if they are continuous variables, then the numerical results are within their limits of uncertainty.

\subsubsection{2g Are the lines of evidence effectively the same quality?}

If the results of the alternative lines of evidence are not the same, then it is necessary to consider their relative quality. The quality of a line of evidence depends on the inherent quality of the models and on the quality and quantity of data used. The inherent quality of the models can be addressed during the generic framework development. The issue of data quality and quantity can be evaluated in a general way (e.g., a model has few data requirements and the data would be available for most sites).

\subsubsection{3g Choose the highest quality estimate}

If the results are different, and if the lines of evidence are of significantly different quality, then the risk estimate from the highest quality line of evidence should be used.

\subsubsection{4g Must multiple modes of action be integrated?}

If the results are effectively the same, or if they are different, but are of the same quality, integrating the results across modes of action of the activity should be considered. That is, it may be important to choose the model that best integrates with other models used to characterize risks from other actions.

\subsubsection{5g Choose the best estimate for integration}

If multiple actions must be integrated, one should choose the method that incorporates mechanisms in a way that best allows integration of the modes of action to estimate the combined risk (Sec. 8.1.3). 


\subsubsection{6g Equivalent estimates}

If the results are effectively the same, or if they are different, but are of the same quality, and if combining multiple actions is not an issue, then one may simply average, weight and average, or in some other appropriate way, combine the results. If the results are so different that averaging is not appropriate, then one of the alternative estimates should be chosen by the DoD manager.

\subsection{3g Multiple Modes of Action}

If the conceptual model for induction of effects contains multiple modes of action, it is necessary to determine whether a combined model is necessary, and, if so, to implement it.

\subsubsection{1g Are there multiple modes of action?}

The conceptual model should indicate whether there are multiple, potentially significant modes of action. For example, in the simple conceptual model for tank maneuvers (Fig. 3.6-2), there are two modes of action for risks to tortoises: 1) direct lethality by crushing, and 2) loss of food due to reduced plant production.

\subsubsection{2g Screen modes of action}

If the effect of only one mode of action is significant to the decision, it is not necessary to consider the combined effects of multiple modes. This may occur for two reasons.

First, if the effects of one mode of action are much greater than the others, the others may be ignored. In nearly all cases, if the expected effects of any mode of action are less than $10 \%$ of those of the most effective mode of action, the former effects may be considered negligible. The increment in risk obtained by including the negligible mode of action would be smaller than the uncertainty concerning the significant stressor. For example, in the case of tank maneuvers and terrestrial herbivores, the effects of food loss may be negligible relative to the direct effects of crushing for a rare species, such as the desert tortoise, which is not trophically limited (Fig. 3.6-2). However, for a species that is limited by food and is able to avoid being crushed, the direct effect may be negligible.

Second, if the effects of one or more individual modes of action are clearly so large as to be unacceptable, then the combined effects need not be estimated. For example, if planned tank maneuvers will kill half of the population of desert tortoises, there is no need to consider the additional effects of food loss.

This decision must be made on the basis of screening assessments, which are assessments performed using simple assumptions or models to determine whether it is appropriate to continue a line of assessment. Conventional methods are available for screening assessments of chemical contaminants (Suter 1995a). Screening methods are not available for most nonchemical stressors, but they will not be needed in most cases because the risk models are not highly complex, and the number of modes of action to be screened is seldom large. Therefore, the screening assessments may be performed by characterizing the risks for each mode of action independently, and then comparing the results to the screening criteria.

\subsubsection{3g Are multiple modes of action significant?}

The results of the screening assessment must be used to determine whether multiple modes of action are potentially significant. This is done by asking:

- Are multiple modes of action potentially significant?

- Is any one mode of action clearly significant acting alone?

If the answer to the first question is "yes" and the second is "no," a combined effects model must be developed. 


\subsubsection{4g Combined Risk Models}

If risks to a particular endpoint from an activity are a product of multiple modes of action, those modes of action must be combined in a common model that estimates the combined risks. This requires analysis and understanding of the modes of action, their underlying mechanisms, and the ways in which they affect the endpoint. It is important to understand the distinction between modes of action and mechanisms of action. A mode of action is a phenomenological description of how an effect is induced. A mechanism of action is a process by which an effect is induced. The mechanism of action is more specific than the mode of action. For example, the mode of action of an agent on a population may be lethality, and its mechanism of action may be crushing, acute narcosis, burning, or starvation. Note that this distinction is a matter of perspective. For example, if the risk estimation is based on reductions in observed abundance following an activity, the phenomenological mode of action is reduction in abundance, and the proximate mechanisms might be mortality, emigration, or reduced reproduction. On the other hand, the phenomenon may be more specific. For example, the observation treated as the mode of action may be of burning (e.g., $x \mathrm{~m}^{2}$ of burned vegetation), and the mechanisms might be wildfire or direct exposure to a pyrophoric munition.

In these cases, the recommended strategy is to define the mode of action and associated mechanisms at as high a level as appropriately possible, given the definition of the endpoint and the available evidence (i.e., exposure-response models). For example, if an endpoint is survival of individuals of an endangered species, then the highest-level relevant mode of action is mortality. One could simply add the number of individuals killed by each mechanism or, if anthropogenic mortality rates are high, calculate the expected number given the probability for each mechanism (allowing for the fact that an organism killed by one mechanism is not available to be killed by others). In this way, risks from different mechanisms can be estimated by a single model by treating them as one mode of action.

Even when multiple modes of action are involved, aggregation of mechanisms to generate higher level modes of action is an important first step. For example, if the endpoint is population abundance of an animal species, aggregation of the various mechanisms of lethality is appropriate. If other modes of action, such as reduced fecundity or increased emigration, were potentially significant, the resulting mortality would be used with those modes in a demographic model to estimate population-level effects. For an example, refer to the combination of toxic effects on survival and fecundity of fish with the effects of harvesting on survival (Barnthouse et al. 1990).

Once the mechanisms of action have been properly aggregated into common modes of action, the combined risk model may be employed. For example, if tank maneuvers kill some tortoises, cause others to emigrate, and reduce fecundity of the remainder, then a simple projection matrix or other demographic model could be used to estimate population reductions.

\section{2g ESTIMATE UNCERTAINTY AND VARIABILITY}

In addition to estimating risks, risk characterizations must estimate uncertainty. The uncertainties associated with exposure and effects characterizations should have been described and estimated in the exposure and effects profiles (Sec. 5.5 and 6.4). If uncertainties are defined for only one of those components of risk, then the uncertainty in the risk estimate is simply the uncertainty in that component. For example, the EPA conventionally estimates uncertainties in exposure, but treats effects models as fully determined (Hansen 1997). If uncertainties are quantified for both exposure and effects, probabilities must be estimated by applying appropriate techniques to the risk estimation model. Uncertainty analysis is discussed in Sec. 12. 


\section{3g RISK DESCRIPTION}

The description of the risk characterization results provides the basis for communicating the risks to decisionmakers and, as appropriate, to other stakeholders and the public. It is contained in the "risk characterization report" that serves as a decision support document and basic risk communication tool (EPA 1998). It should address the following points:

- Nature of effects

- Intensity of effects

- Spatial scale

- Temporal scale

- Rates and results of recovery

- Causes of the effects

- Probability of the effects

- Nature and concordance of alternative lines of evidence

- Degree of confidence in the estimates

- Other issues known to be important to decisionmakers or stakeholders.

In a generic framework, this section should provide the assessor with a format for the document. The document should be designed to stand alone as an expression of the risk. It is not a substitute, however, for actually documenting the full methods and results of the activity assessment. A user or reviewer of the assessment must have the option of digging deeply into the assessment in order to judge its quality and validity. 


\section{8i. ACTIVITY-SPECIFIC RISK CHARACTERIZATION: IMPLEMENTATION}

When implementing the risk characterization for a training or testing program, the assessor must begin by deciding which of the alternative lines of evidence should be implemented. The potential alternative lines of evidence should have been evaluated during the activity-specific problem formulation. Implementation of the exposure and effects characterizations for the activity, however, may have revealed that some lines of evidence that appeared appropriate and feasible during the activity-specific problem formulation were, in fact, not feasible, or that the data for implementation were not available, or were weak. The assessor must then use the exposure and effects characterization results to estimate risks for each selected line of evidence. If more than one line of evidence is used, a weight of evidence analysis must be performed to generate a final risk estimate. If there are multiple modes of action for the activity and endpoint, the risk estimates for each mode of action must be combined. A model for the combined effects should be specified in the generic framework for the activity. If the effects of the activity are an increment to the effects of prior occurrences of the training or testing activity, both the incremental effects and the cumulative effects should be reported. This may be done by simply adding the effects, if the cumulative effects are additive, or by estimating risks using a cumulative exposure estimate (Sec. 5i). This process should result in a risk estimate to be reported, along with its uncertainties, in a risk description. Even if the activity is part of a multi-activity training or testing program, the risk description should be generated for each activity, so that decisionmakers and stakeholders can understand what the individual activities, acting alone, contribute to the risk. 


\section{INTEGRATED RISK CHARACTERIZATION}

This section describes how to characterize the risks posed by all activities comprising a training, testing, or resource management program. This risk characterization integrates the results for component activities (Sec. 8) to estimate the risk to an endpoint receptor, based on the combined effects of all stressors generated by all activities. The results could be used to determine the acceptability of a program or to compare alternative program plans.

\subsection{MORE THAN ONE ACTIVITY?}

Some training or testing programs consist of only one activity. If that is the case, and if there are no resource management activities with which the training or testing activity must be integrated, the result of the activity-specific risk characterization serves as the risk characterization for the program.

\subsection{PROGRAMMATIC RISK ESTIMATION}

Programmatic risk estimation integrates the effects of all activities within a training or testing program on an endpoint to provide an estimate of the combined risk. This may, depending on the circumstances, be a simple matter of adding up the risks or it may require a complex analysis. The logic of this section is based on a process of attempting to combine the risks of each activity with the minimum of additional analysis. That is, first determine if the risks are independent and can be simply summarized; if not, can they be added or otherwise combined; if not, can their exposures be added and the risks recalculated or, if not, one must re-estimate the risks of the combined activities. This depends on the spatial and temporal relationships among the activities and the modes of action of the stressors. Hence, the complexity of the programmatic risk estimation depends on the extent to which the environmental effects of the component activities are independent or interactive.

\subsubsection{Spatial and Temporal Overlap?}

If there is no spatial and temporal overlap of the activities, the risks of the individual activities need not be integrated. The spatial and temporal extent of the program should have been defined in the programmatic problem formulation (Sec. 3.4). However, the temporal and spatial extent estimated for planning purposes may be inaccurate by subsequent assessment activities. The spatial and temporal extent of each activity should have been defined in the activity-specific problem formulation in terms of a core area and influence area (Sec. 4.3). At this step in the process, these areas must be combined across activities to determine whether the activities are spatially or temporally independent. Independence may be determined by asking the following questions.

- Are the activities performed in the same or overlapping areas?

- If not, is there significant interaction between the endpoint populations or ecosystems in the areas?

- If the areas are the same or overlap, are the activities performed at different times?

- If so, is there sufficient time for ecological recovery between activities?

\subsubsection{Summarize Independent Effects}

If the activities are spatially or temporally independent, the programmatic risk characterization consists of a summary of the risks from all component activities.

\subsubsection{Multiple Activities Significant?}

If the effect of only one activity is significant to the decision, it is not necessary to consider the combined effects of multiple activities. This simplification may occur for two reasons. 
First, if the effects of one activity are much greater than the others, the others may be ignored. In nearly all cases, if the expected effects of any activity are less than $10 \%$ of those of the most effective activity, it may be considered negligible. Since ecological risks cannot be estimated with a precision of $10 \%$ or less, the increment in risk obtained by including the negligible activity would be smaller than the uncertainty concerning the significant activity. For example, in the case of a training program involving vehicles and foot troops, the effects of trampling are likely to be negligible relative to crushing by vehicles, especially for a rare species such as desert tortoise, which is not limited by food or space.

Second, if the effects of one or more individual modes of action are clearly so large as to be unacceptable, then the combined effects need not be estimated. For example, if vehicles are expected to kill half of the population of desert tortoises, there is no need to consider the additional effects of foot troops.

\subsubsection{Report Risk of Major Effect}

If all but one activity have insignificant effects, the risks from the one significant activity should be reported as the risks of the program (Sec. 8.1).

\subsubsection{Are Effects Additive?}

The effects of activities on an endpoint are additive if they have the same mode of action (Sec. 8.1.3) and if they are linear with respect to the endpoint within the range of exposure. To return to the tortoise example, driving tracked vehicles, driving wheeled vehicles, and firing live artillery rounds all have acute lethality as a mode of action. If the number of mortalities is a small proportion of the population, the proportional reduction in the population size is a linear function of the number killed. However, if a significant proportion of the population is killed, nonlinearities due to factors such as reduced ability of survivors to find mates must be considered.

Similarly, for the destruction of food resources as a mode of action, effects may be linear within limited ranges of exposure. There may be three ranges: (1) from zero loss of food plants to the point at which food resources become limiting, (2) from the initiation of food limitations to the point at which the area is abandoned or the tortoise starves, and (3) the range in which abandonment or starvation occur. However, if combined exposures to stressors with that mode of action are in a different range from the exposures for individual activities, the effects are not additive.

Effects additivity models are also used for chemical mixtures when each chemical is lethal to some proportion of the exposed population (or has some other binary effect), but their mechanisms of action are independent.

\subsubsection{Sum Effects}

If effects are additive, the programmatic risk estimation is performed by adding the effects estimated for each individual activity and reporting that estimate as the effect of the combined activities. In some cases, it will be necessary to consider that you cannot cause the same effect twice. For example, a tortoise killed by one activity is not available to be killed by others. Therefore, if there are two activities $a$ and $b$, the number of tortoises killed by both is:

$$
N_{k}=p_{a} N+p_{b} N-p_{a b} N,
$$

where

$N_{k}$ is the number killed

$p_{a}$ is the probability of being killed by activity $a$

$N$ is the number of individuals in the exposed population 
$p_{b}$ is the probability of being killed by activity $b$

$p_{a b}$ is the probability of being killed by activities $a$ and $b$.

\subsubsection{Are Exposures Additive?}

If effects are not additive, exposures may still be additive if the mode or mechanism of action is the same, so that the same exposure-response model is applicable. This may be the case if, as discussed above, the mode of action is the same, but the exposure-response model is nonlinear. It is also the case if exposures of the agents acting independently are insufficient to cause the effect of concern through the specified mechanism of action, but the combined exposure is sufficient. One such case is combined exposures to chemicals with a common mechanism of action. For example, multiple narcotic chemicals, occurring together at sublethal doses, may be fatal. The models for these combined toxic effects are termed "concentration additivity models." In either case, one would add the exposures and run the exposure-response model for that mode or mechanism of action using the total exposure. If exposures are not additive, then they must be modeled separately.

\subsubsection{Add Exposures and Estimate Effects}

If the mode of action is the same, but the effects are not additive due to nonlinearities, add the exposures and run the exposure-response model for that mode of action using the total exposure. Report the estimate as the effect of the combined activities.

\subsubsection{Mechanistically Model Combined Effects}

If there are multiple modes of action among the activities, a model must be selected that incorporates all modes of action. As discussed in Sec. 8.1.3.4, this requires a consideration of the mechanisms underlying the mode of action and selection of an appropriate mechanistic level for the model. In general, selection of higher levels results in simpler models that are more easily implemented. However, interactions among the exposure and response processes generally require a deeper level of mechanistic description. For example, combined effects of contaminants and harvesting on fish populations have been modeled using relatively simple demographic models by assuming that the contaminants only reduce survivorship and fecundity, and harvesting only effects survivorship (Barnthouse et al. 1990). However, if one wishes to estimate the effects of compensatory processes, such as reduced interference among survivors and increased food supply, one must incorporate mechanisms that affect individual fish (Rose et al. 1993). In many cases, the extent to which these more deeply mechanistic models can be applied is limited by both scientific knowledge and by lack of needed sitespecific information. Note that simple threshold models or empirical models that may have provided the best estimate of risks of an individual activity are likely to be inappropriate for the estimation of combined effects. Hence, in cases of multiple lines of evidence for a given activity, the logic of selecting a best line of evidence will need to be reapplied (Sec. 8.1.2).

\subsection{COMBINED UNCERTAINTY AND VARIABILITY}

In addition to estimating risks, risk characterizations must estimate the uncertainty. The uncertainties associated with each activity should have been described and estimated in the activityspecific risk characterizations (Sec. 8.2). Component uncertainties must be appropriately combined, however, and new uncertainties will be introduced by the integration of risks from multiple activities (Sec. 9.2). Uncertainty analysis is discussed in Sec. 12.

\subsection{COMBINED RISK DESCRIPTION}

The description of the risk characterization results provides the basis for communicating the risks to decisionmakers and, as appropriate, to other stakeholders and the public. It is contained in the "risk 
characterization report" that serves as a decision support document and basic risk communication tool (EPA 1998). It should address the following points:

- Nature of effects

- Intensity of effects

- Spatial scale

- Temporal scale

- Rates and results of recovery

- Causes of the effects

- Probability of the effects

- Nature and concordance of alternative lines of evidence

- Degree of confidence in the estimates

- Other issues known to be important to decisionmakers or stakeholders

This document should stand alone as an expression of the risk. However, it is not a substitute for actually documenting the full methods and results of the activity assessment. A user or reviewer of the assessment must have the option of digging deeply into the assessment in order to judge its quality and validity. 


\section{RISKS TO THE MISSION}

Implementing decisions tends to create opportunities in some directions while limiting opportunities in others. Management of natural resources on military training ranges may do both, for the military mission. Proper management helps maintain long-term sustainable ranges, promote realism and safety, and fulfill DoD responsibilities as a steward of the nation's resources. Restrictions and risks to the military mission can also occur as a result of good management and, in different ways, as a result of lack of management. Ongoing interactions between military activities and natural environments will always generate certain risks to the military mission and to the environment. The output of ecological risk assessment and its role in supporting management decisions is important as input to an assessment of risks to the military mission due to features of the natural environment, management, policies, and requirements relating to that environment. The output of the ecological risk assessment (Sec. 8) can be linked to an assessment of risks to the military mission and incorporated into military planning for longterm mission sustainability.

Military mission risk assessment per se is not currently a part of this ecological risk assessment framework. However, this section discusses how output from this framework could be incorporated into assessment of risk to the military mission.

\subsection{CATEGORIES OF INFORMATION NEEDED}

To estimate risks to the military mission due to environmental impacts, direct and indirect, on the training and testing ranges - the DoD's "environmental infrastructure" - several categories of information are needed.

\subsubsection{Environmental Characteristics Needed for the Military Mission}

One category of information needed concerns specific knowledge about what kinds of environmental characteristics and functions must or should be available, to support high-quality training or testing of a specific military activity. (And alternatively, what characteristics should be absent in order to prevent adverse impact on the military mission.) Some aspects of this kind of information may be extrapolated from military documents, such as field training manuals and tactical doctrine, as well as a few existing scientific studies. But data and understanding about military needs for specific environmental characteristics and functions are very far from complete, at least on the scientific side of this partnership in understanding. Here is an example list of desirable environmental characteristics for land ranges:

- good trafficability and accessibility

- desirable tactical features, such as camouflage and concealment

- absence of safety hazards

- absence of uncontrolled dust and erosion damage

- sufficient contiguous and accessible space to conduct the necessary military activities

- low risk of flooding and ground saturation

- minimal restrictions on military use

- realistic environment to prepare for real-world engagements.

\subsubsection{Obtaining data about military environmental needs}

There is an overall lack of comprehensive sources for understanding military environmental training and testing needs. Trafficability models have been developed by the U.S. Army Engineer Waterways Experiment Station in Mississippi. Aspects of training land design, such as tactical concealment islands, have been studied and implemented by the U.S. Army Construction Engineering Research Laboratory in Illinois. A prioritized list of conservation "User Requirements" for each of the 
military services is compiled in the Andrulis Report (Feige and Strauss 1994) and in subsequent updates. Some information and models are being developed under the Army's Integrated Training Area Management (ITAM) program, in particular, models for carrying capacity of land for military training. Land use pattern analysis, using remotely sensed data and analytical capabilities of a GIS, could provide some indications of preferred landscape features for training. The most essential source of information is likely to be the expert judgment of military operations and logistics personnel, range managers, and installation natural resource managers and engineers.

\subsubsection{Risk to Environmental Variables}

A second category of information needed is the set of risk estimations or assessments regarding impacts on the environmental variables due to military activity. We must obtain a picture of risk to the needed or useful aspects of the environment for military purposes, before estimating the risk to the mission that could be caused by damage to these aspects. This process of ecological risk assessment has been discussed in detail in the preceding sections.

\subsubsection{Risks to Mission Due to Social Valuation of Environmental Characteristics}

In addition to environmental characteristics that support the military mission directly, there are also issues of risk to the military mission due to the social values placed on certain environmental characteristics, such as endangered species and their habitats. The presence of an endangered species may have no direct effect on training or testing. Legislation, regulations, and the demands created by social values related to that species, however, can severely constrain the execution of the military training and testing mission. Thus, the estimation of present or future risks to the military mission, due to the need to protect socially valued characteristics of environments impacted by DoD activities, is an important component of risk estimation from the military mission perspective.

This information is best obtained through the ecological risk assessment process previously described in this report. By evaluating, assessing, and predicting risk to socially valued environmental characteristics and functions, it should be possible to extrapolate the risk posed to the military mission due to DoD's stewardship responsibilities to the nation.

\subsection{RISK MITIGATION FACTORS}

It is at this stage of the process that mitigation, prevention, and proactive management measures should also be factored in. Such measures can be applied from the environmental management side, the military operations and logistics side, or the coordinated application of both. The most efficient way of analyzing risk mitigation effects is to use current and under-development decision support tools for managing the natural environment, operations, and logistics relating to training and testing activities. Risk to valued environmental variables can be estimated, using risk assessment approaches and tools, both with and without the application of mitigation and proactive management measures. From this analysis, the relative risks to the military mission can be derived and compared for different scenarios of mitigation due to ecological management and to military operations management.

\subsection{INCORPORATING ECOLOGICAL RISKS INTO MILITARY PLANNING}

In the long term, conjunctions will be necessary between the tools and approaches for estimating and managing environmental risk, and for managing the military mission and risk to the mission. This trend is already beginning to take place, as military operations personnel and land managers become familiar with and make use of similar tools and information sources, such as GISs, remotely sensed data, and decision support systems. Newer versions of some decision support systems for military purposes incorporate environmental factors, such as the Army's Range Facility Management and Support System (RFMSS), though they do not handle risk per se. The Army's ITAM program has, as one of its functions, 
the identification of natural resource maintenance needs to support optimal long-term military use of training areas. However, this is not based on a formal risk assessment process. These are movements in the right direction and provide potential opportunities for more explicit linkages between ecological risk assessment and military planning in the future.

Creating and implementing such a conjunction is beyond the scope of the current project. This project will conceptualize how such conjunctions need to function, and will identify and discuss potential linkages with selected military decision support tools currently used or under development. This project will also provide recommendations that could be applied to DoD policy development directed toward risk-based management of the environment founded upon an understanding of risk to the military mission. 


\section{INTERFACE WITH RISK MANAGEMENT}

The risk assessment task is not complete when the risks have been characterized. In cases where a substantial portion of the assessment has been performed by contractors, the assessors must assure that the results are understood by the DoD manager, and may assist in incorporating the results into any decision support system the DoD manager uses. This section describes how to perform that activity. In particular, it briefly describes existing decision support systems and their links to risk assessment.

\subsection{PRESENTING RESULTS TO THE DoD RISK MANAGER}

The ecological risk assessment framework must be designed to present the output and results from the assessment process in a clear, comprehensible and useful format for use by the DoD risk manager. The results must be described in terms that are familiar to risk managers and that are comparable to outputs from other, related processes and systems that the risk manager may be relying upon.

\subsection{USING THE RESULTS IN DECISIONMAKING}

The risk manager must then take the risk assessment results and apply them to the decision to be made, using whatever decisionmaking processes are considered appropriate. These results must be formatted in a way to make them as useful as possible with the current and planned decision support processes and tools in DoD.

Decisionmaking processes are currently in place in DoD. For example, the test ranges have a procedure for decisionmaking and documentation for NEPA requirements. This procedure will be examined for developing a compatible format of the results from the MERAF risk assessment with the test range procedure.

\subsection{USING THE RESULTS AS INPUT TO DECISION SUPPORT SYSTEMS}

A decisionmaking process also may include use of computerized decision-support tools. For example, the U.S. Army Construction Engineering Research Laboratory is developing the Land Management System (LMS), which includes a set of modeling and decision-support tools applicable for land management of land-based military installations. One existing decision-support tool is PRISM (Planning and Resource Integration Stewardship Modules), developed at the U.S. Army Construction Engineering Research Laboratory. PRISM uses a series of GIS overlays to display natural resources data to assist in decisionmaking regarding specific installations. The Integrated Dynamic Landscape Analysis and Modeling System (IDLAMS) is a decision support system that includes a module to examine risks to threatened and endangered species.

Most of the decision support models or tools contain default values. These default values may be particularly useful for estimating results when specific information is incomplete.

The outputs from the MERAF must be formatted to be compatible as input to existing and planned decision support systems in use by DoD. 


\section{TREATMENT OF UNCERTAINTY ${ }^{1}$}

The explicit consideration of uncertainty is a hallmark of risk assessment. Guidance for environmental impact assessments also requires that uncertainties be described (CEQ 1986). However, formal uncertainty analyses are seldom performed for NEPA documents. The analysis of uncertainty has several benefits:

- It permits the estimation of risks that effects will be greater than expected.

- It eliminates the need for conservative assumptions.

- It permits the proper propagation of multiple uncertainties through an assessment model.

- It identifies the major sources of uncertainty for prioritization of monitoring and research.

- It makes decisionmakers and others aware of the potential limitations of the assessment results.

The EPA has recently issued guidance for analysis of uncertainty in human and ecological risk assessments. That guidance, reproduced in Box 1, contains conditions for acceptance of uncertainty analyses in risk assessments.

\section{Box 1. Conditions for Acceptance (Hansen 1997)}

When risk assessments using probabilistic analysis techniques (including Monte Carlo analysis) are submitted to the Agency for review and evaluation, the following conditions are to be satisfied to ensure high quality science. These conditions, related to the good scientific practices of transparency, reproducibility, and the use of sound methods, are summarized here and explained more fully in the Attachment, "Guiding Principles for Monte Carlo Analysis."

1. The purpose and scope of the assessment should be clearly articulated in a "problem formulation" section that includes a full discussion of any highly exposed or highly susceptible subpopulations evaluated (e.g., children, the elderly). The questions the assessment attempts to answer are to be discussed and the assessment endpoints are to be well defined.

2. The methods used for the analysis (including all models used, all data upon which the assessment is based, and all assumptions that have a significant impact upon the results) are to be documented and easily located in the report. This documentation is to include a discussion of the degree to which the data are representative of the population under study. Also, this documentation is to include the names of the models and software used to generate the analysis. Sufficient information is to be provided to allow the results of the analysis to be independently reproduced.

3. The results of sensitivity analyses are to be presented and discussed in the report. Probabilistic techniques should be applied to the compounds, pathways, and factors of importance to the assessment, as determined by sensitivity analyses or other basic requirements of the assessment.

4. The presence or absence of moderate to strong correlations or dependencies between the input variables is to be discussed and accounted for in the analysis, along with the effects these have on the output distribution. (Cont'd)

1 This section is adapted and condensed from Suter 1998. 


\section{Box 1. Conditions for Acceptance (Cont'd).}

5. Information for each input and output distribution is to be provided in the report. This includes tabular and graphical representations of the distributions (e.g., probability density function and cumulative distribution function plots) that indicate the location of any point estimates of interest (e.g., mean, median, 95th percentile). The selection of distributions is to be explained and justified. For both the input and output distributions, variability and uncertainty are to be differentiated where possible.

6. The numerical stability of the central tendency and the higher end (i.e., tail) of the output distributions are to be presented and discussed.

7. Calculations of exposures and risks using deterministic (e.g., point estimate) methods are to be reported if possible. Providing these values will allow comparisons between the probabilistic analysis and past or screening level risk assessments. Further, deterministic estimates may be used to answer scenario specific questions and to facilitate risk communication. When comparisons are made, it is important to explain the similarities and differences in the underlying data, assumptions, and models.

8. Since fixed exposure assumptions (e.g., exposure duration, body weight) are sometimes embedded in the toxicity metrics (e.g., Reference Doses, Reference Concentrations, unit cancer risk factors), the exposure estimates from the probabilistic output distribution are to be aligned with the toxicity metric.

The following are commentaries on the conditions relative to ecological risk assessment of training and testing, and several additional points to consider in performing uncertainty analyses.

1. The purpose of the assessment should be clearly defined in the problem formulation for the training or testing program as discussed in Sec. 3.1.

2. The disclosures called for in this condition are good practice whether or not probabilistic methods are used. Note that the term "population" should be taken to mean the population of entities to be represented by the data (i.e., a statistical population, not a biological population). For example, the population may be a population of soil samples. Also, the documentation called for in this and other conditions should be presented in appendices or in some other manner that does not diminish the readability of the risk characterization report.

3. The results of sensitivity analyses are only one of the "basic requirements of the assessment" that should determine which of the parameters should be defined as probability distributions.

- If a probabilistic analysis is replacing an analysis that included uncertainty factors or conservative assumptions, the parameters to which those factors or assumptions were applied should be treated as uncertain.

- If the regulators, resource managers, or other stakeholders have expressed concern that an uncertain parameter may affect the outcome of the assessment, treat the parameter as uncertain in the analysis.

- If the probabilistic analysis is performed in support of a planning decision, the parameters relevant to the decision must be treated as uncertain. For example, if the analysis is performed to aid development of a sampling and analysis plan, those parameters that may be measured must be treated as uncertain.

- The EPA's statement that parameters determined to be influential by the sensitivity analysis should be treated as uncertain, should be applied when other more relevant criteria are not applicable. If this requirement were given primacy, it could cause one to select parameters, such as the explosive force of a bomb, that are not significantly uncertain or variable and are not relevant to the decision. 
4. Moderate to strong correlations among parameters are common in risk models, and, if ignored, they inflate the output distribution. Body weights, feeding rates, and water consumption rates, for example, are all highly correlated. If correlations are believed to occur but cannot be estimated from available data, perform Monte Carlo simulations with correlations set to zero and set to high, but plausible, values to determine their importance and presence (Burmaster and Anderson 1994).

5. These requirements for disclosure of the nature of the distributions are appropriate. The demand for plots of both probability density and cumulative density functions for each input and output parameter seems excessive, but they do provide different views of the functions that give a more complete understanding than either alone. The tabular presentation should include the following:

- Name of the parameter

- Units of the parameter

- If variable, with respect to what does it vary?

- Formula for the distribution of variability

- Basis for the distribution of variability

- If uncertain, what sources of uncertainty are considered?

- Formula for the uncertainty distribution

- Basis for the distribution of uncertainty.

Distributions that are developed ad hoc may require considerable explanation of their bases. These may include the data from which they are derived or the elicitation techniques for expert judgments, plus an explanation of how the data or judgments relate to the assumed sources of the variability or uncertainty. If the expert judgments of individuals are used to derive distributions, any information or logic that went into the judgment should be described as fully as possible. Burmaster and Anderson (1994) indicate that a typical justification for a distribution would require five to ten pages.

6. This condition refers to the changes in the variability of the moments of the output distribution as the number of iterations of a Monte Carlo analysis increases. Most software packages provide criteria for termination of the analysis based on the stability of the output distribution. This condition specifies that the stability of both central tendency and an extreme should be noted and recorded. The specific mention of "high end" is based on the EPA's implicit assumption that only exposure will be probabilistically analyzed. For effects distributions, the lower extreme is of greater interest.

7. In general, one should perform a deterministic analysis using realistic or best estimate values for the parameters, and a conservative deterministic analysis may be performed using conservative assumptions and parameters, such as those favored by a regulatory agency. In some cases, discrepancies among conservative point estimates, best point estimates, regulatory estimates, and medians of probabilistic results will be quite large. The causes of these differences should be explained.

8. Reference doses, slope factors, or equivalent have not been provided by the EPA for ecological risk assessment. Rather, ecological risk assessors must assure themselves and others that the expressions of exposure and effects are concordant and make sense on an individual basis, given the site conditions and assessment endpoint. Note that for distributions, this requirement goes beyond simple checking of units. One must consider the element that is distributed, as well as to what it is distributed.

The following additional considerations are not among the EPA conditions, but are also important to assure the quality of probabilistic ecological risk assessments.

9. To the extent possible, use empirical information in the derivation of distributions (Burmaster and Anderson 1994). 
10. The correlation matrix must have a feasible structure. For example, if parameters $a$ and $b$ are both strongly positively correlated with $c$, then they cannot be strongly negatively correlated with each other (Ferson 1996).

11. Multiple instances of the same variable in a model must be assigned the same value in an iteration of a Monte Carlo analysis (Ferson 1996). This is a particular problem in stepwise analyses in which different components of risk are estimated by separate simulations.

12. Care must be taken to avoid nonsensical values of input and output distributions. For example, negative values should be prevented for parameters such as concentrations or body weights, herbivore consumption rates should not exceed plant production rates, and contaminant concentrations should not exceed a million parts per million. This can be prevented by truncation or, in some cases, by the appropriate selection of the distribution.

13. In general, for parameter uncertainty, it is more important to treat the parameters correctly (e.g., do not treat variables as constant), next most important is to treat the magnitude of variability or uncertainty correctly, and least important (but still not trivial) is to treat the form of the distribution correctly (e.g., triangular versus normal).

14. For species sensitivity distributions, distributions of exposure measures, and similar parametric distribution functions, report goodness of fit statistics and prediction intervals as minimum estimates of model uncertainty.

15. In general, model uncertainty cannot be well or reliably estimated, but it should be acknowledged. The acknowledgment should list specific issues in model selection or design that are potentially important sources of error. That list should include issues regarding initial disagreement among the parties, or issues in which there is no consensus in ecological risk assessment practice.

16. To the extent possible, specify for model assumptions whether they introduce an identifiable bias. Examples include:

- Assuming $100 \%$ bioavailability of contaminants introduces a conservative bias

- Assuming independent toxic effects introduces an anticonservative bias

- Assuming additive toxic effects introduces a conservative bias

- Assuming the chemical occurs entirely in its most toxic form introduces a conservative bias

- Assuming the most sensitive species of a small number of test species is representative of sensitive species in the field introduces a conservative bias.

Note that a bias does not mean that there is a consistent direction of error in every case. Cases of synergistic effects, for example, would not have the conservative bias associated with assuming additive toxicity. The bias is real, however, because such synergistic effects are much less common than less-than-additive effects.

17. Quantification of uncertainties due to model assumptions is difficult, but should be performed to the extent possible. For example, the uncertainty from assuming the chemical occurs entirely in its most toxic form can be bounded by presenting results for the least toxic form. 


\section{GLOSSARY}

The following terms and definitions are common to ecological risk assessment, but may not be familiar to the reader. Those that were developed for this report are underlined. All others are from EPA 1998.

Activity - An activity is defined as a standard procedure for accomplishing a discrete task. The procedure is repeated over time and at different places to accomplish the training or testing task with different military units. Activities comprise elements described in field manuals and training circulars, and may be components of mission essential task lists (METLs). Alternatively, an assessment may be conducted on a nonmilitary program, such as soil erosion control. An erosion control program could include activities such as leveling, terracing, constructing checkdams, revegetation, and constructing sediment traps or settling ponds.

Agent - Any physical, chemical, or biological entity that can induce an adverse response (synonymous with stressor).

Assessment endpoint - An explicit expression of the environmental value that is to be protected. An assessment endpoint includes both an ecological entity and specific attributes of that entity. For example, salmon are a valued ecological entity; reproduction and population maintenance of salmon form an assessment endpoint.

Characterization of ecological effects - A portion of the analysis phase of ecological risk assessment that evaluates the ability of a stressor to cause adverse effects under a particular set of circumstances.

Characterization of exposure - A portion of the analysis phase of ecological risk assessment that evaluates the interaction of the stressor with one or more ecological entities. Exposure can be expressed as co-occurrence or contact, depending on the stressor and ecological component involved.

Combined risk - Risks that result from the multiple activities or multiple stressors generated by an activity jointly acting at a location or within the range of an endpoint receptor. (See cumulative risk.)

Community - An assemblage of populations of different species within a specified location in space and time.

Comparative risk assessment - A process that generally uses an expert judgment approach to evaluate the relative magnitude of effects, and sets priorities among a wide range of environmental problems. Some applications of this process are similar to the problem formulation portion of an ecological risk assessment in that the outcome may help select topics for further evaluation and help focus limited resources on areas having the greatest risk reduction potential. In other situations, a comparative risk assessment is conducted more like a preliminary risk assessment. For example, EPA's Science Advisory Board used expert judgment and an ecological risk assessment approach to analyze future ecological risk scenarios and risk management alternatives.

Concentration additivity models - Models in which the exposures of the agents acting independently are insufficient to cause the effect of concern through the specified mechanism of action, but the combined exposure is sufficient. One would add the exposures and run the exposure-response model for that mode or mechanism of action using the total exposure. As an example, multiple narcotic chemicals, occurring together at sublethal doses, may be fatal. If exposures are not additive, then they must be modeled separately.

Conceptual model - The conceptual model describes a series of working hypotheses of how the stressor might affect ecological entities. The conceptual model also describes the ecosystem potentially at risk, the relationship between measures of effect and assessment endpoints, and exposure scenarios. 
Core area/time - Bounds on the areas and time periods in which direct exposure to training and testing activities occur.

Cumulative distribution function (CDF) - Cumulative distribution functions are particularly useful for describing the likelihood that a variable will fall within different ranges of $\mathrm{x}$. F(x) (i.e., the value of $\mathrm{y}$ at $\mathrm{x}$ in a CDF plot) is the probability that a variable will have a value less than or equal to $\mathrm{x}$.

Cumulative risk - Risks that result from the repeated occurrence of an activity at a location or within the range of an endpoint receptor. (See combined risk.)

Direct effect - An effect where the stressor acts on the ecological component of interest itself, not through effects on other components of the ecosystem (synonymous with primary effect; compare with definition for indirect effect).

Disturbance - Any event or series of events that disrupts ecosystem, community, or population structure and changes resources, substrate availability, or the physical environment.

Ecoepidemiology - The process of determining the magnitude and causes of effects on nonhuman organisms and communities observed in the field.

Ecological entity - A general term that may refer to a species, a group of species, an ecosystem function or characteristic, or a specific habitat. An ecological entity can be one component of an assessment endpoint.

Ecological risk assessment - The process that evaluates the likelihood that adverse ecological effects may occur or are occurring as a result of exposure to one or more stressors.

Ecosystem - The biotic community and abiotic environment within a specified location in space and time.

Endpoint area/time - Bounds on the area and time periods in which the endpoint species or communities are present.

Environmental Impact Statement (EIS) - Assessments are required under the National Environmental Policy Act (NEPA) to fully evaluate environmental effects associated with proposed major Federal actions. Like ecological risk assessments, EIS typically require a "scoping process" analogous to problem formulation, an analysis by multidisciplinary teams, and a presentation of uncertainties (CEQ 1986).

Exposure - The contact or co-occurrence of a stressor with a receptor.

Exposure profile - The product of characterization of exposure in the analysis phase of ecological risk assessment. The exposure profile summarizes the magnitude and spatial and temporal patterns of exposure for the scenarios described in the conceptual model.

Exposure scenario - A set of assumptions concerning how an exposure may take place, including assumptions about the exposure setting, stressor characteristics, and activities that may lead to exposure.

Functional groups - Functional groups may be defined in two ways (Catovsky 1998). First, they may be defined in terms of the functional response of the group to a prior process. For example, highly mobile animals are functionally distinct in their response to wildfires from less mobile organisms that cannot avoid being burned. Second, they may be defined in terms of their functional effect on a subsequent process. For example, herbaceous plants, but not trees or algae, function as input to herbivory by voles. These types of functional groups have been termed functional response groups and functional effects groups, respectively (Catovsky 1998). 
Hazard assessment - This term has been used to mean either (1) evaluating the intrinsic effects of a stressor or (2) defining a margin of safety or quotient by comparing a toxicologic effects concentration with an exposure estimate.

Indirect effect - An effect where the stressor acts on supposing components of the ecosystem, which in turn have an effect on the ecological component of interest (synonymous with secondary effect; compare with definition for direct effect).

Influence area/time - Bounds on the areas and time periods in which the indirect consequences of training and testing activities occur.

Influence models - Conceptual models that show environmental influences on the properties of endpoint receptors. Andrewartha and Birch (1984) provide an excellent discussion of these models and several examples. They recommend defining influences as resources, predators, malentities, and mates. Resources include food, water, nutrients, and habitat features such as nest sites. Predators include hunters, pathogens, parasites, and conventional predators. Malentities include toxicants, extreme weather, fire, and tillage. Mates include both the presence of potential mates and the actual availability for reproduction that may be influenced by weather, habitat conditions or human disturbance.

Intensity - The measure of magnitude of the stressor that renders it capable of inducing an effect. That is, it is the concentration, pressure, or other characteristic that determines how strongly the stressor acts on the receptor.

Lines of evidence - Information derived from different sources or by different techniques that can be used to interpret and compare risk estimates. While this term is similar to the term "weight of evidence," it does not necessarily imply assignment of quantitative weightings to information.

Lowest observed adverse effect level (LOAEL) - The lowest level of a stressor evaluated in a test that causes statistically significant differences from the controls.

Maximum acceptable toxic concentration (MATC) - For a particular ecological effects test, this term is used to mean either the range between the NOAEL and the LOAEL or the geometric mean of the NOAEL and the LOAEL for a particular test. The geometric mean is also known as the chronic value (CV).

Measure of ecosystem and receptor characteristics - A measurable characteristic of the ecosystem or receptor that is used in support of exposure or effects analysis.

Measure of effect - A measurable ecological characteristic that is related to the valued characteristic chosen as the assessment endpoint.

Measure of exposure - A measurable stressor characteristic that is used to help quantify exposure.

Measurement endpoint - See "measure of effect."

Mechanism of Action - The process by which an effect is induced. It is more specific than the mode of action. For example, the mode of action of an agent on a population may be lethality and its mechanism of action may be crushing, acute narcosis, burning, or starvation.

Median lethal concentration (LC50) - A statistically or graphically estimated concentration that is expected to be lethal to $50 \%$ of a group of organisms under specified conditions.

Mode of Action - A phenomenological description of how an effect is induced. See Mechanism of Action.

No observed adverse effect level (NOAEL) - The highest level of a stressor evaluated in a test that does not cause statistically significant differences from the controls. 
Population - An aggregate of individuals of a species within a specified location in space and time.

Primary effect - An effect where the stressor acts on the ecological component of interest itself, not through effects on other components of the ecosystem (synonymous with direct effect; compare with definition for secondary effect).

Probability density function (PDF) - Probability density functions are particularly useful in describing the relative likelihood that a variable will have different particular values of $\mathrm{x}$. The probability that a variable will have a value within a small interval around $\mathrm{x}$ can be approximated by multiplying $\mathrm{f}(\mathrm{x})$ (i.e., the value of $y$ at $x$ in a PDF plot) by the width of the interval.

Receptor - The ecological entity exposed to the stressor.

Recovery - The rate and extent of return of a population or community to a condition that existed before the introduction of a stressor. Due to the dynamic nature of ecological systems, the attributes of a "recovered" system must be carefully defined.

Relative risk assessment - A process similar to comparative risk assessment. It involves estimating the risks associated with different stressors or management actions. To some, relative risk connotes the use of quantitative risk techniques, while comparative risk approaches more often rely on expert judgment. Others do not make this distinction.

Risk characterization - A phase of ecological risk assessment that integrates the exposure and stressor response profiles to evaluate the likelihood of adverse ecological effects associated with exposure to a stressor. The adversity of effects is discussed, including consideration of the nature and intensity of the effects, the spatial and temporal scales, and the potential for recovery.

Risk characterization report - A report that serves as a decision support document and basic risk communication tool. It should address the following points: nature and intensity of effects, spatial and temporal scales, rates and results of recovery, causes and probabilities of effects, nature and concordance of alternative lines of evidence, degree of confidence in the estimates, and other issues known to be important to parties involved in decision and implementation.

Secondary effect - An effect where the stressor acts on supporting components of the ecosystem, which in turn have an effect on the ecological component of interest (synonymous with indirect effects; compare with definition for primary effect).

Source - An entity or action that releases to the environment or imposes on the environment a chemical, physical, or biological stressor or stressors.

Source term - As applied to chemical stressors, the type, magnitude, and patterns of chemical(s) released.

Stressor - Any physical, chemical, or biological entity that can induce an adverse response (synonymous with agent).

Stressor-response profile - The product of characterization of ecological effects in the analysis phase of ecological risk assessment. The stressor response profile summarizes the data on the effects of a stressor and the relationship of the data to the assessment endpoint.

Trophic levels - A functional classification of taxa within a community that is based on feeding relationships (e.g., aquatic and terrestrial green plants comprise the first trophic level and herbivores comprise the second). 


\section{REFERENCES}

40 CFR 1500, National Environmental Policy Act (NEPA) 1969, Pub. L. 91-190, 42 U.S.C. 4321-4347, January 1, 1970, as amended by Pub. L. 94-52, July 3, 1975, Pub. L. 94-83, August 9, 1975, and Pub. L. 97-258, § 4(b), Sept. 13, 1982.

Andrewartha, H.G. and L.C. Birch. 1984. The Ecological Web. University of Chicago Press, Chicago.

Barnthouse, L.W. and J. Brown. 1994. Conceptual model development: Ch. 3. Ecological Risk Assessment Issue Papers. EPA/630/R-94/009. U.S. Environmental Protection Agency (EPA), Washington, D.C.

Barnthouse, L.W. and G.W. Suter, II. 1986. User's manual for ecological risk assessment. ORNL-6251. Oak Ridge National Laboratory, Oak Ridge, TN.

Barnthouse, L.W., G.W. Suter II, and A.E. Rosen. 1990. "Risks of toxic contaminants to exploited fish populations: Influence of life history, data uncertainty, and exploitation intensity." Environ. Toxicol. Chem. 9:297-311.

Barnthouse, L.W., G.W. Suter II, A.E. Rosen, and J.J. Beauchamp. 1987. "Estimating responses of fish populations to toxic contaminants." Environ. Toxicol. Chem. 6:811-824.

Bartell, S.M., R.H. Gardner, and R.V. O'Neill. 1992 . Ecological Risk Estimation. Lewis Publishers, Ann Arbor, Michigan.

Blacker, S. and D. Goodman. 1994a. Risk-based decision making: An integrated approach for efficient site cleanup. Environ. Sci. Technol. 28:466A-470A.

Blacker, S. and D. Goodman. 1994b. "Risk-based decision making: Case study: application at a superfund cleanup." Environ. Sci. Technol. 28:471A-477A.

Burmaster, D.E. and P.D. Anderson. 1994. "Principles of good practice for the use of Monte Carlo techniques in human health and ecological risk assessment." Risk Analysis 14(4):477-481.

Calabrese, E.J. and L. A. Baldwin. 1994. "A toxicological basis to derive a generic interspecies uncertainty factor." Environ. Health Perspect. 102:14-17.

Canter, L. and B. Sadler. 1997. A tool kit for effective EIA practice - review of methods and perspectives on their application. Environmental and Groundwater Institute, Norman, OK.

Carpenter, R.A. 1995. "Risk assessment.” Impact Assessment 13:153-187.

Catovsky, S. 1998. "Functional groups: clarifying our use of the term." Bulletin of the Ecological Society of America 79(1):126-127.

CEQ (Council on Environmental Quality). 1986. "Regulations for implementing the procedural provisions of the National Environmental Policy Act." 40 CFR Parts 1500-1508. U.S. Government Printing Office, Washington, D.C.

Craighead, J.J. and F.C. Craighead. 1956. Hawks, Owls and Wildlife. Dover Pub., Inc., New York.

Deputy Undersecretary of Defense (Environmental Security) [DUSD(ES)] 1994. Memorandum, subj: Implementation of Ecosystem Management in the DoD, 08 Aug 1994, DUSD(ES)/EQ-CO, Washington, D.C.

Efroymson, R.A., W.W. Hargrove, M.J. Peterson, D.S. Jones, W.H. Rose, L. Pater, K. Reinbold, and G.W. Suter II. 2001b. "Demonstration of the Military Ecological Risk Assessment Framework (MERAF): Apache-Longbow-Hellfire Missile Test at Yuma Proving Ground.” ORNL/TM- 
2001/211. Oak Ridge National Laboratory, Oak Ridge, TN.

Efroymson, R.A., W.H. Rose, S. Nemeth, G.W. Suter II. 2000. "Ecological risk assessment framework for low-altitude overflights by fixed-wing and rotary-wing military aircraft." ORNL/TM-2000/289. Oak Ridge National Laboratory, Oak Ridge, TN.

Efroymson, R.A., and G.W. Suter II. 2001. "Ecological risk assessment framework for low-altitude aircraft overflights: II. Estimating effects on wildlife." Risk Analysis 21:263-274.

Efroymson, R.A., G.W. Suter II, W.H. Rose, and S. Nemeth. 2001a. "Ecological risk assessment framework for low-altitude aircraft overflights: I. Planning the analysis and estimating exposure." Risk Analysis 21:251-262.

EPA. 1992. "Framework for ecological risk assessment." EPA/630/R-92/001. Risk Assessment Forum, Washington, D.C.

EPA. 1994. "Guidance for the data quality objectives process." EPA QA/G-4. Quality Assurance Management Staff, U.S. EPA, Washington, D.C.

EPA. 1998. "Guidelines for ecological risk assessment.” EPA/630/R-95/002F. Risk Assessment Forum, Washington, D.C.

Feige, E. and T. Strauss. 1994. U.S. Army Environmental Research and Development Requirements: Final Report. Andrulis Research Corporation, Arlington, VA.

Ferson, S. 1996. "Automated quality assurance checks on model structure in ecological risk assessment." Human \& Ecological Risk Analysis 2(3):558-569.

Fletcher, J.S., F.L. Johnson and J.C. McFarlane. 1990. "Influence of greenhouse versus field testing and taxonomic differences on plant sensitivity to chemical treatment." Environ. Toxicol. Chem. 9:769776.

Gell-Mann, M. 1994. The Quark and the Jaguar: Adventures in the Simple and the Complex. W.H. Freeman, New York.

Hansen, F. 1997. "Policy for use of probabilistic analysis in risk assessment at the U.S. Environmental Protection Agency.” http://www.epa.gov/ncea/mcpolicy.htm. U.S. EPA, Washington, D.C.

Health Council of the Netherlands. 1991. "Quality parameters for terrestrial ecosystems and sediments: a selection of practical ecotoxicologic assessment methods." 1991/17E.

Host, G.E., R.R. Regal and C. E. Stephan. 1991. "Analysis of acute and chronic data for aquatic life." PB93-154748. U.S. EPA Report, Duluth, MN.

Jorgensen, S.E. 1994. Fundamentals of Ecological Modeling. Elsevier, Amsterdam.

Karr, J.R., K.D. Fausch, P.L. Angermeier, P.R. Yant, and I.J. Schlosser. 1986. Assessing biological integrity in running waters; a method and its rationale. Illinois Natural History Survey, Special Pub. 5. Champaign, Illinois.

Liddle, M.J. 1975. "A selective review of the ecological effects of human trampling on natural ecosystems.” Biol. Conserv. 7:17-36.

NRC (National Research Council). 1983. Risk assessment in the federal government: Managing the process. National Academy Press, Washington, D.C.

NRC (National Research Council). 1993. Issues in Risk Assessment. National Academy Press, Washington, D.C. 
O'Neill, R.V., D.L. DeAngelis, J.B. Waide, and T.F.H. Allen. 1986. A Hierarchical Concept of Ecosystems. Princeton University Press, New Jersey.

Plafkin, J.L., M.T. Barbour, K.D. Porter, S.K. Gross, and R.M. Hughes. 1989. "Rapid bioassessment protocols for use in streams and rivers: benthic macroinvertebrates and fish." EPA/444/4-89-001. U.S. EPA, Washington, D.C.

Pokras, M.A., A.M. Karas, J.K. Kirkwood, and C.J. Sedgewick. 1993. "An introduction to allometric scaling and its uses in raptor medicine": pp. 211-224. P.T. Redig, J.E. Cooper, J.D. Remple and D.B. Hunter (eds.), Raptor Biomedecine. U. Minnesota Press, Minneapolis.

Reinbold, K., W. Hodge, H. Balbach, M. Chawla, R. Sundell, T. Kvitek, D. Schaeffer, M. Day, K. Paige, A. Krzysik, B. Dickson, M. Stees, and L. Pater. 1997 draft. Impact Assessment for Management of Department of Defense Natural and Cultural Resources: A Survey of Current Methods, Data, Policies, and Practices with Recommendations for Future Development. U.S. Army Construction Engineering Research Laboratories, Champaign, IL.

Rose, K.A., J.H. Cowan, E.D. Houde, and C.C. Coutant. 1993. "Individual-based modeling of environmental quality effects on early life-stages of fishes: a case study using striped bass." American Fisheries Society Symposium 14:125-145.

Sample, B.E., D.M. Opresko, and G.W. Suter II. 1996. "Toxicological benchmarks for wildlife." ES/ER/TM-86/R3. Oak Ridge National Laboratory, Oak Ridge, TN.

Science Policy Council. 1997. "Guidance on Cumulative Risk Assessment. Part 1. Planning and Scoping." http://www.epa.gov/ord/spc/cumrisk2.htm. U.S. EPA, Washington, DC.

Sigal, L.L. 1993. "Sourcebook for the environmental assessment (EA) process." EPA 300-B-93-007. U.S. EPA, Washington, D. C.

Suter, G.W. II. 1989. "Ecological endpoints": pp 2-1-2-28. W. Warren-Hicks, B.R. Parkhurst and J.S. Baker (eds.), Ecological Assessment of Hazardous Waste Sites: A Field and Laboratory Reference Document. EPA 600/3-89/013. Corvallis Environmental Research Laboratory, Corvallis, OR.

Suter, G.W. II. 1993. Ecological Risk Assessment. Lewis Publishers, Boca Raton, FL.

Suter, G.W. II. 1995a. "Guide for performing screening ecological risk assessments at DOE facilities." ES/ER/TM-153. Lockheed Martin Energy Systems, Inc., Oak Ridge, TN.

Suter, G.W. II. 1995b. "Endpoints of interest at different levels of biological organization": pp 35-48. J. Cairns, Jr., and B.R. Niederlehner (eds.), Ecological Toxicity Testing: Scale, Complexity, and Relevance. Lewis, Boca Raton, FL.

Suter, G.W. II. 1996a. "Guide for developing conceptual models for ecological risk assessment." ES/ER/TM-186. Oak Ridge National Laboratory, Oak Ridge, TN.

Suter, G.W. II. 1996b. "Risk characterization for ecological risk assessment of contaminated sites." ES/ER/TM-200. Oak Ridge National Laboratory, Oak Ridge, TN.

Suter, G.W. II. 1998. "Guidance for treatment of variability and uncertainty in ecological risk assessment.” ES/ER/TM-228. Oak Ridge National Laboratory, Oak Ridge, TN.

Suter, G.W. II, J.W. Gillett, and S. Norton. 1994. "Characterization of exposure": Ch. 3. Ecological Risk Assessment Issue Papers. EPA/630/R-94/009. U.S. Environmental Protection Agency, Washington, D.C. 
Suter, G.W. II, D.S. Vaughan, and R.H. Gardner. 1983. "Risk assessment by analysis of extrapolation error, a demonstration for effects of pollutants on fish." Environmental Toxicology and Chemistry 2:369-378.

Troyer, M.E. and M.S. Brody. 1994. "Managing ecological risks at EPA: issues and recommendations for progress.” EPA/600/R-94/183. U.S. EPA, Washington, D.C.

Van Straalen, N.M. and C.A.J. Denneman. 1989. "Ecotoxicological evaluation of criteria." Ecotoxicology and Environmental Safety. 18:241-251.

Wentsel, R.S., T.W. La Point, M. Simini, R.T. Checkai, D. Ludwig, and L.W. Brewer. 1996. "Tri-service procedural guidelines for ecological risk assessments.” 19960924 088. U.S. Army, Edgewood Research, Development, and Engineering Center, Aberdeen Proving Ground, MD. 


\section{CHARTS}

Numbers of/in the charts correspond to section numbers in the text.

Numbers followed by "g" indicate a generic process (i.e., it does not contain site-specific or program-specific information).

Numbers followed by "i” indicate implementation of a generic process (i.e., it contains site-specific and program-specific information).

Numbers followed by " $r$ " indicate activity-specific framework tasks for resource management purposes. 


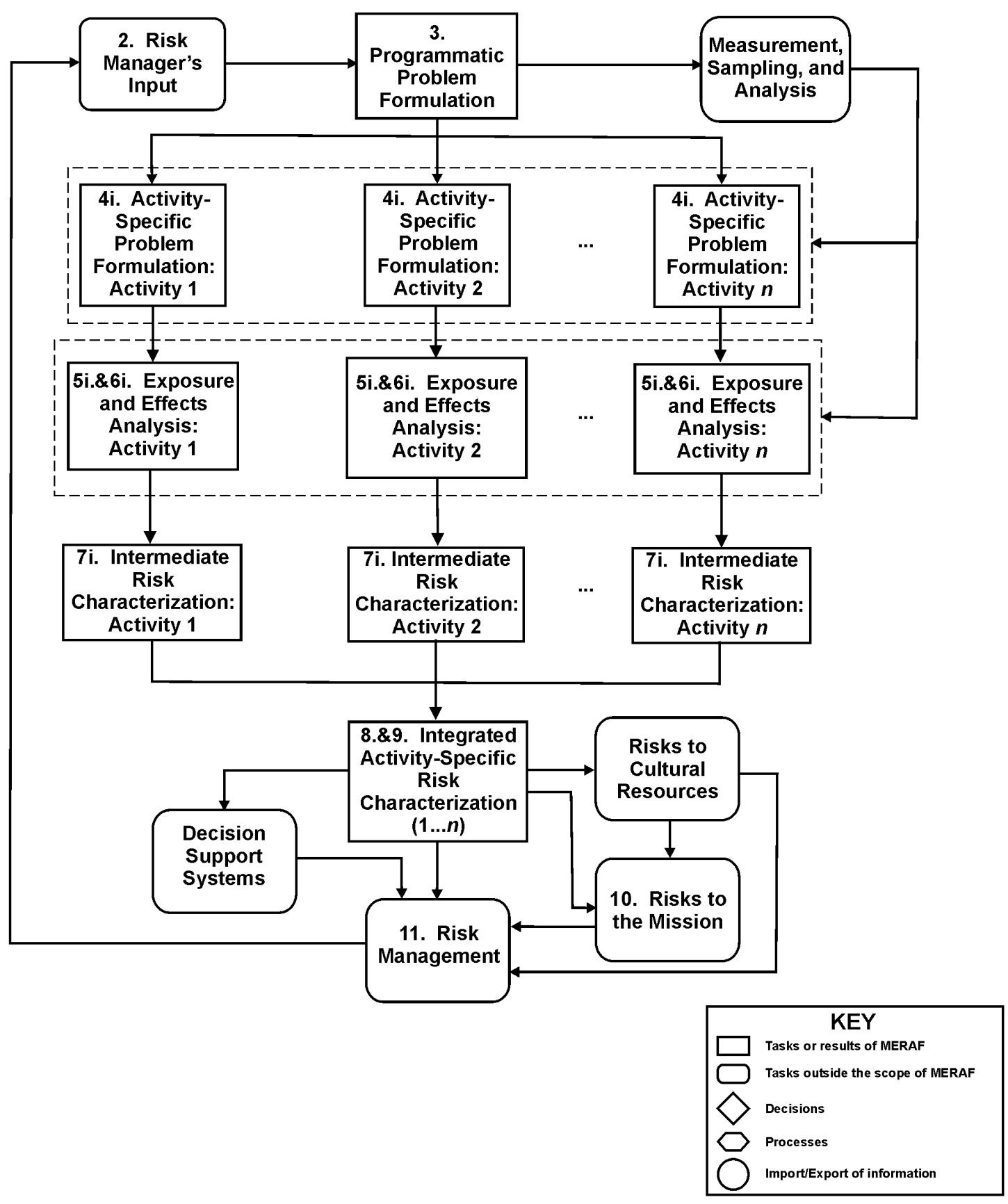

Chart 1. Military Ecological Risk Assessment Framework (MERAF). 


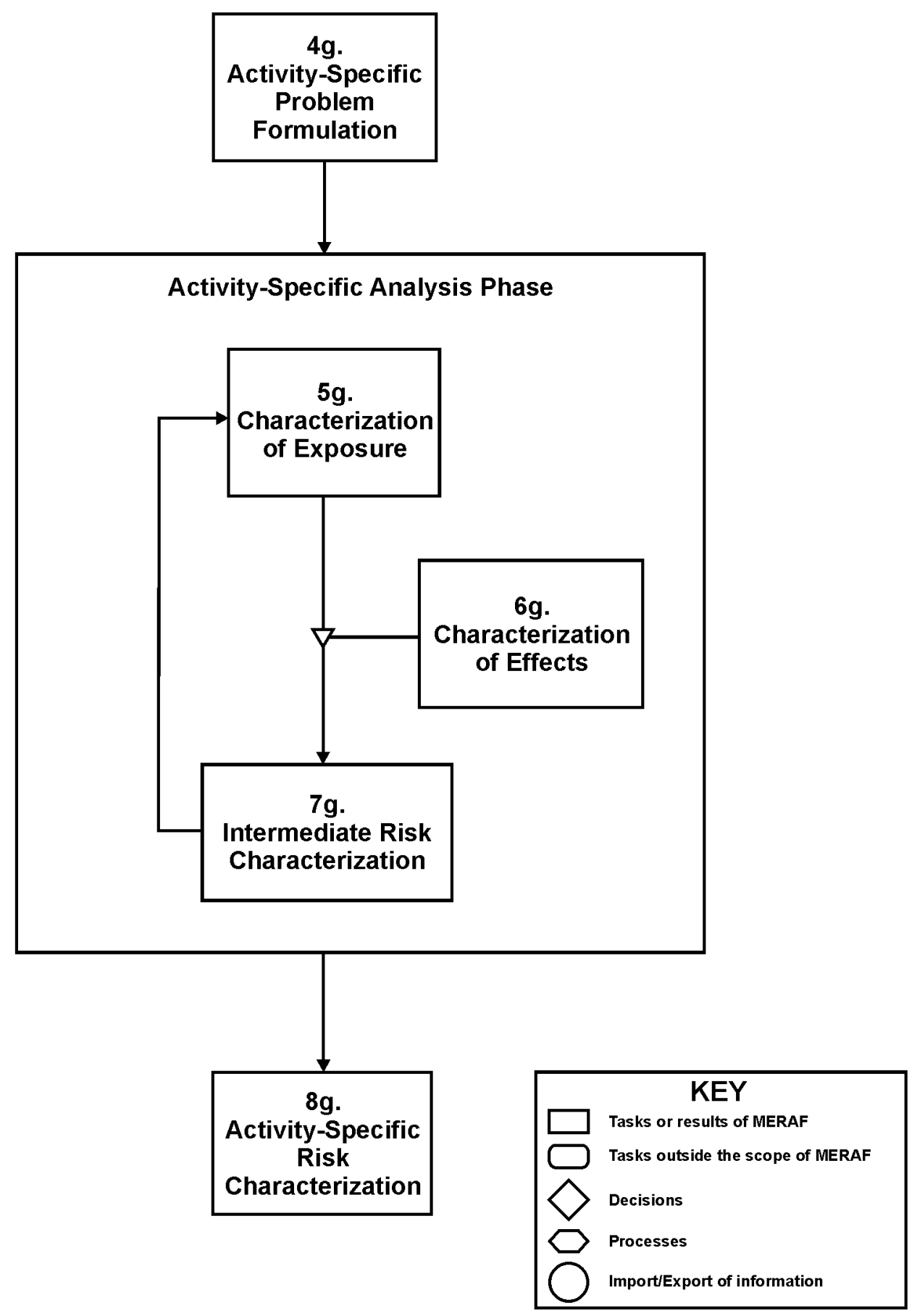

Chart 1g. Generic Activity-Specific Framework Structure. 


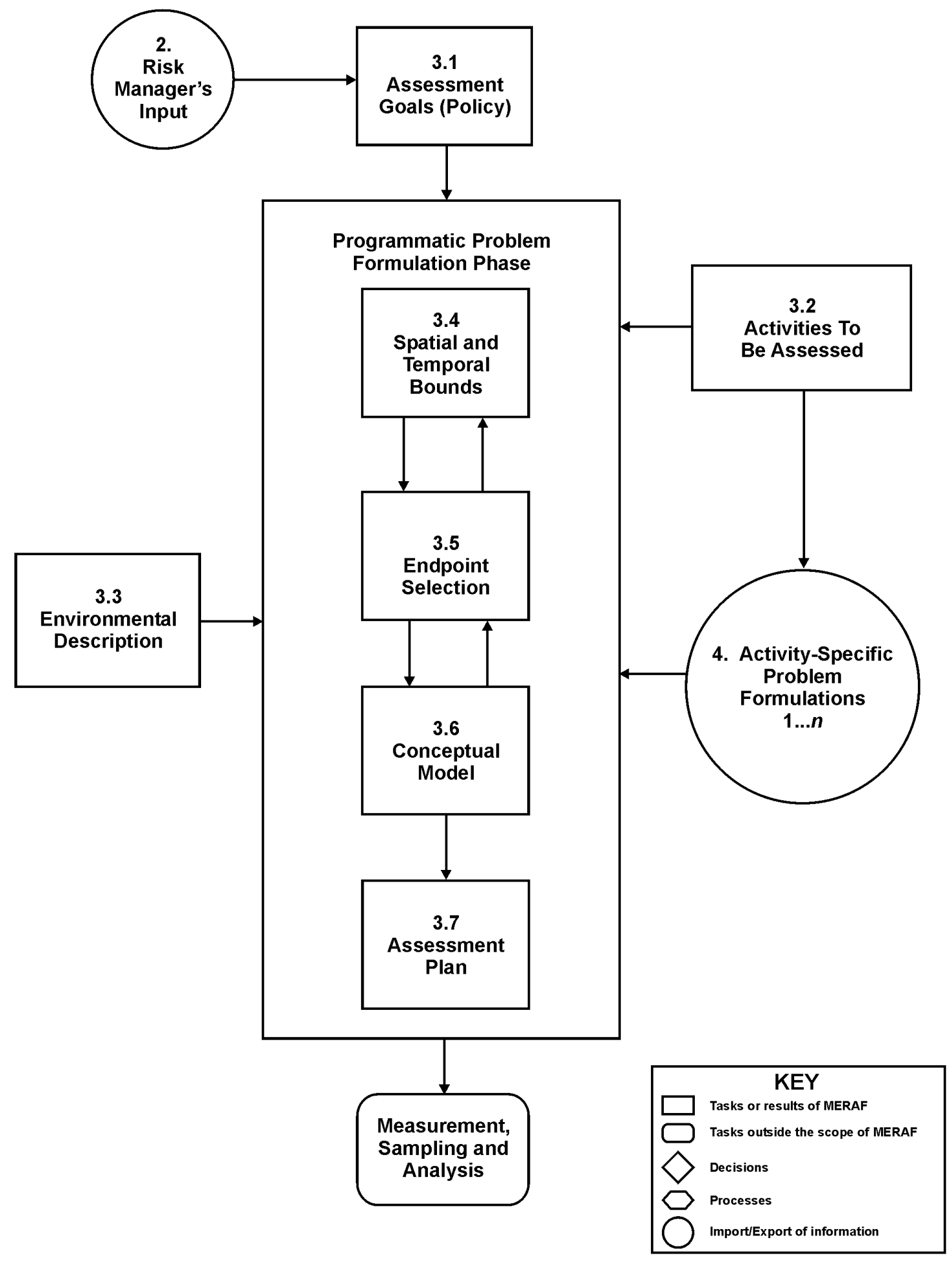

Chart 3. Programmatic Problem Formulation. 


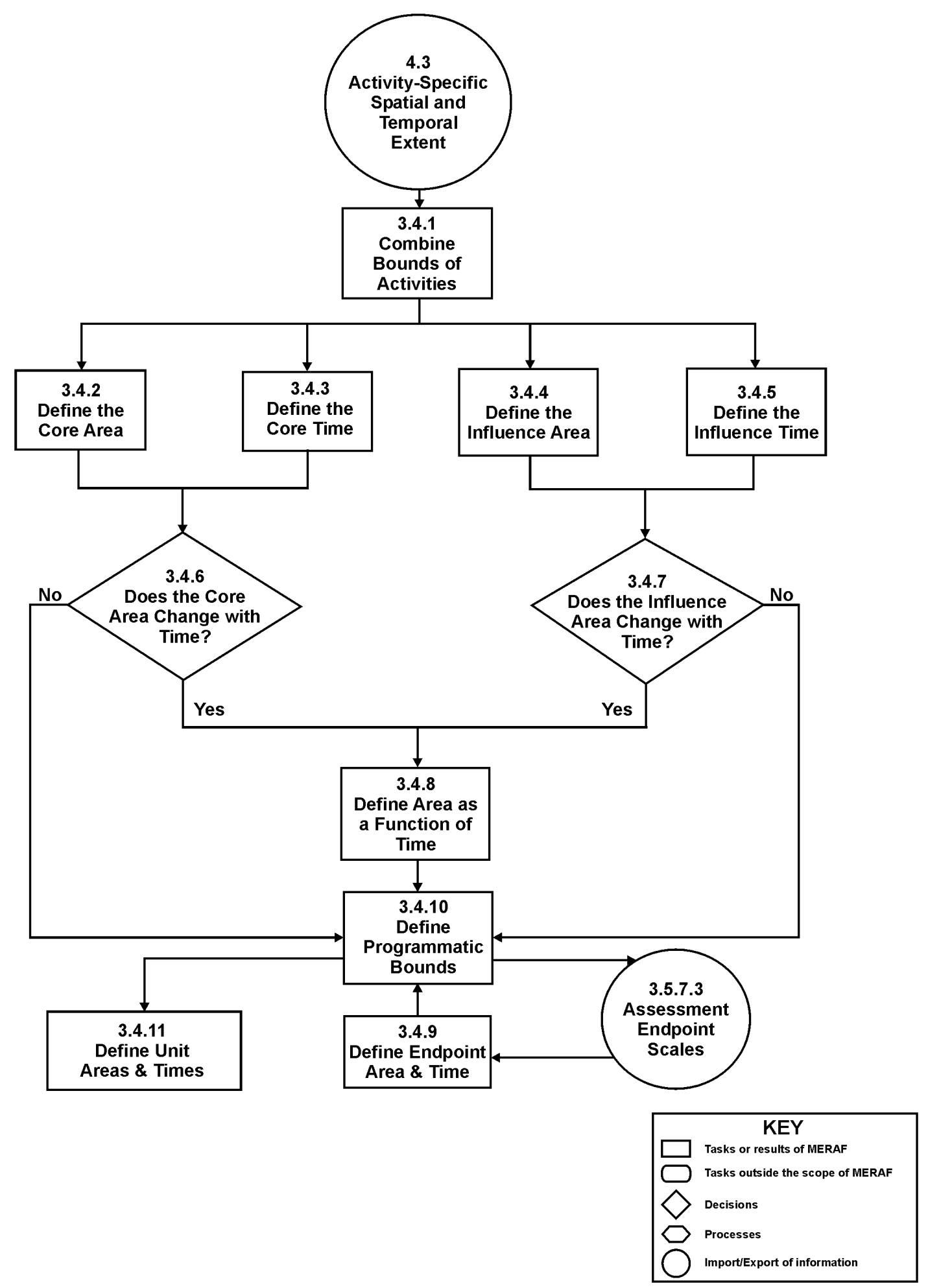

Chart 3.4. Programmatic Spatial and Temporal Bounds. 


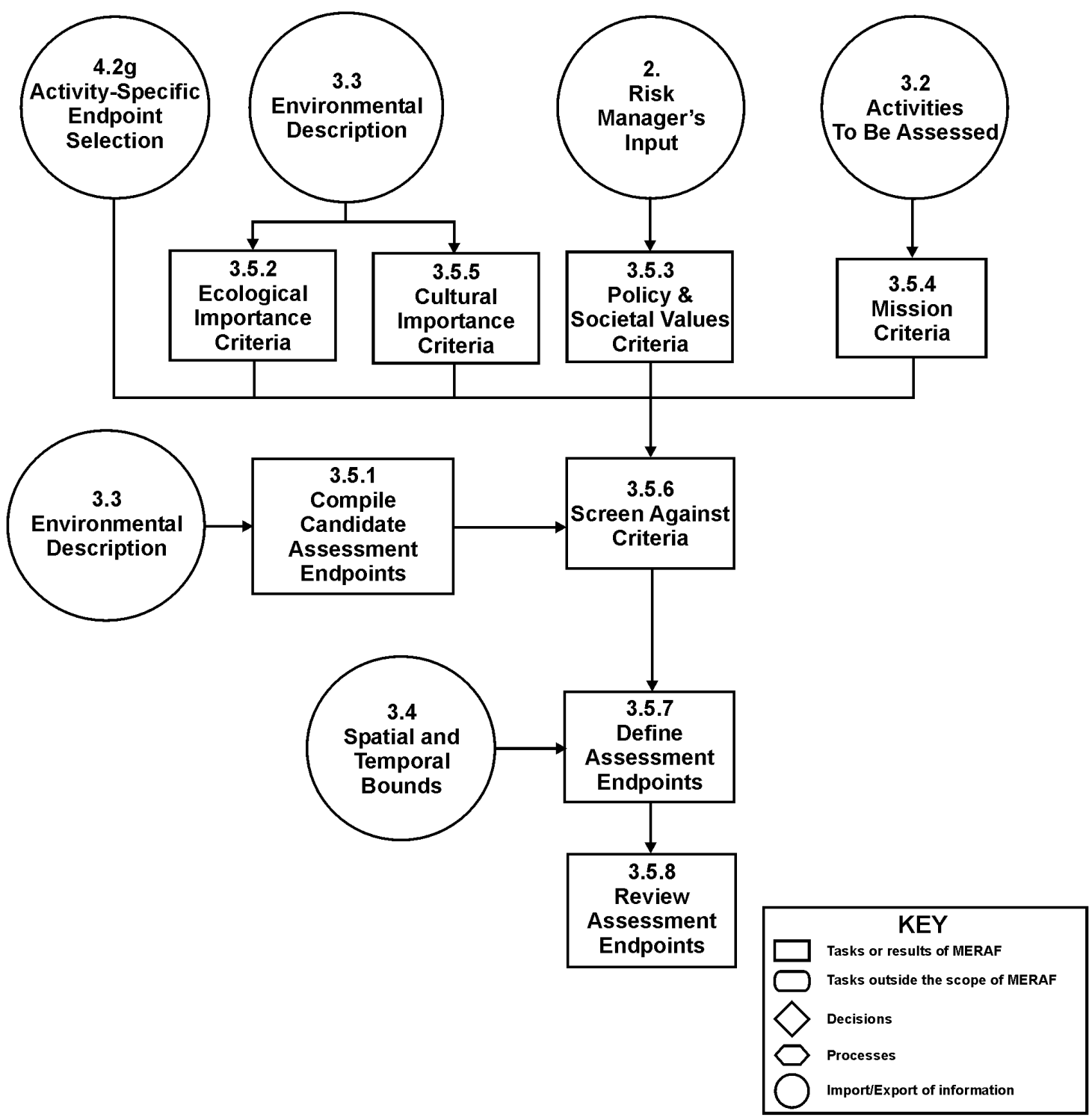

Chart 3.5. Programmatic Assessment Endpoint Selection. 


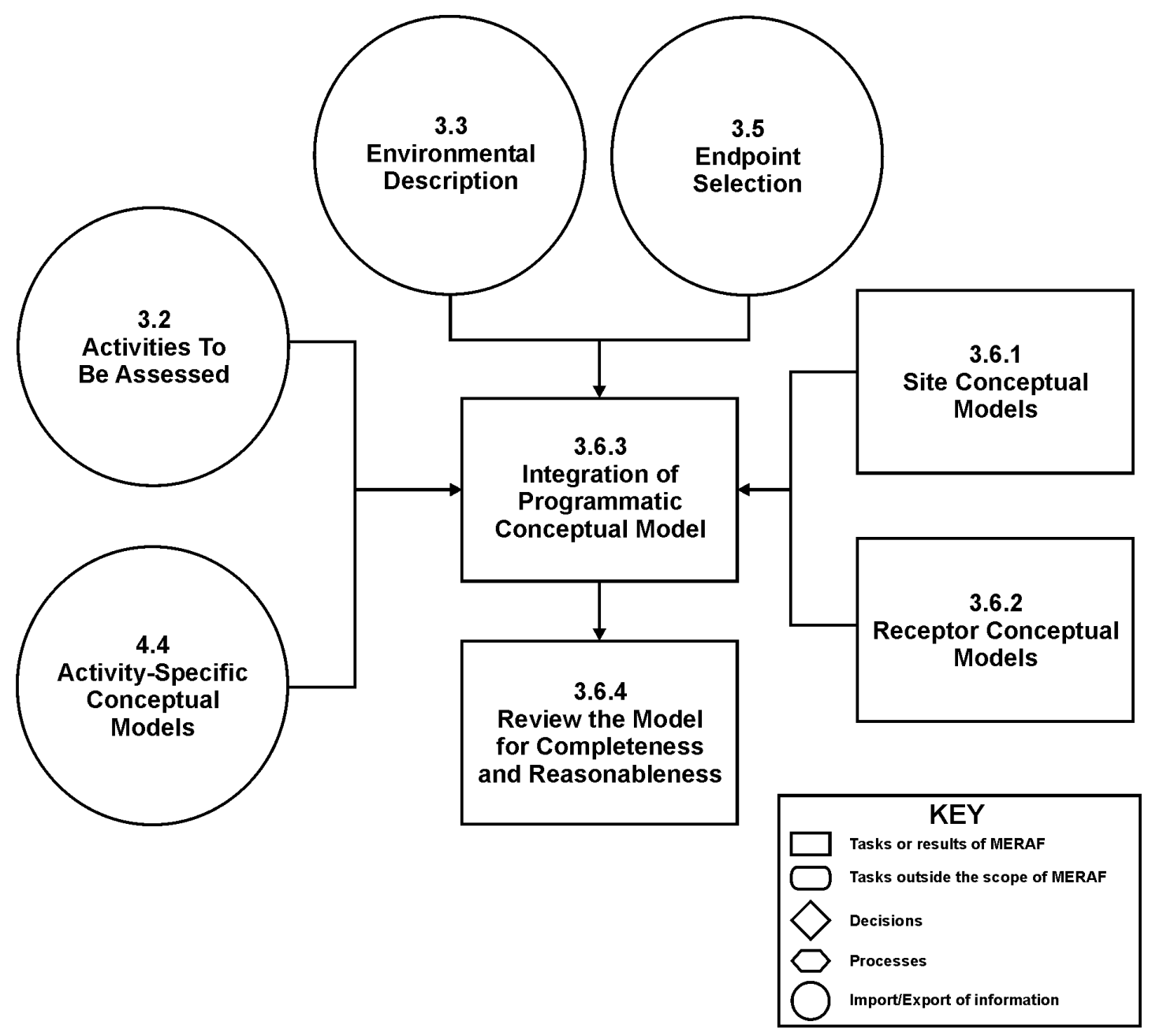

Chart 3.6. Programmatic Conceptual Model Development. 


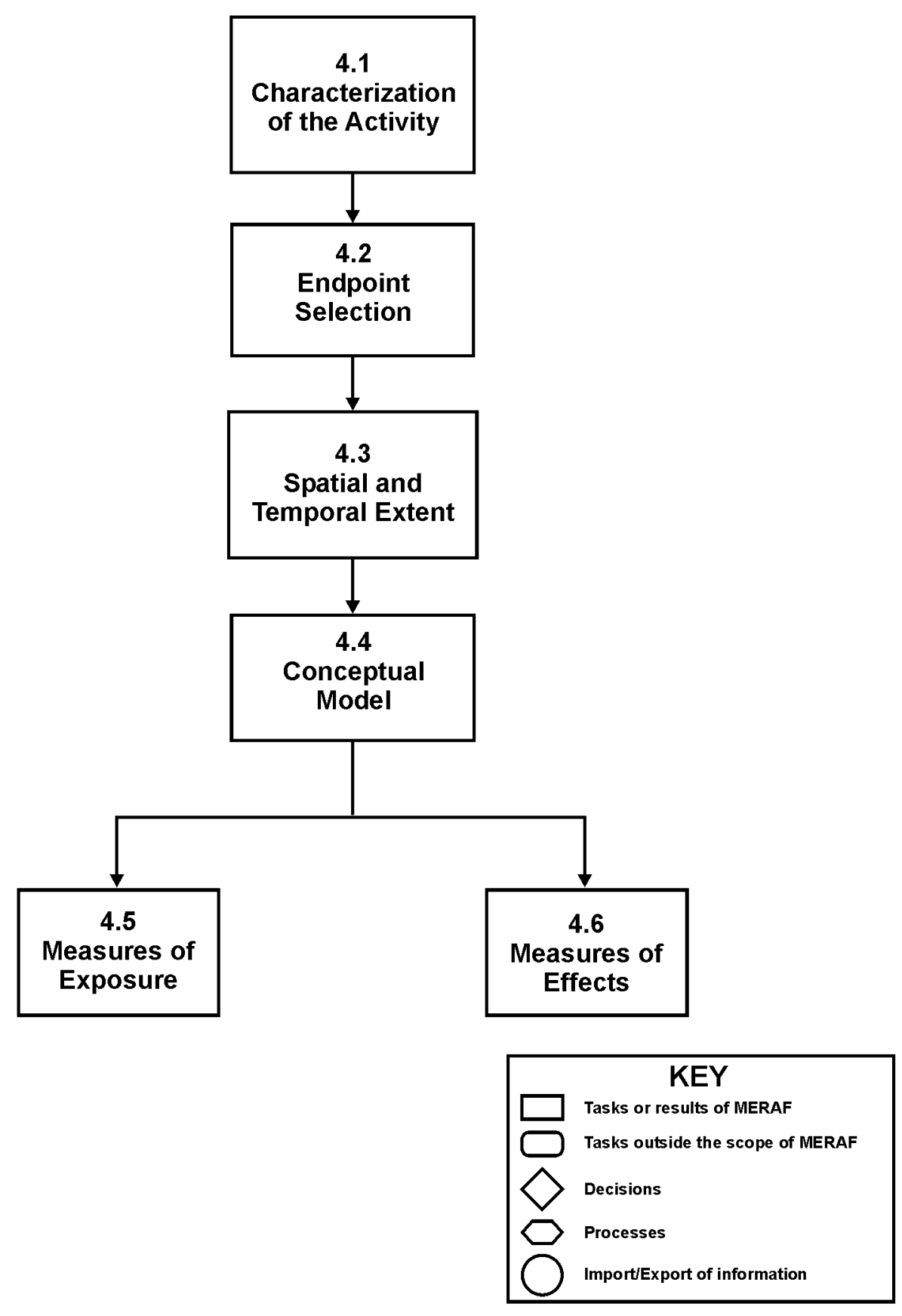

Chart 4. Activity-Specific Problem Formulation. 


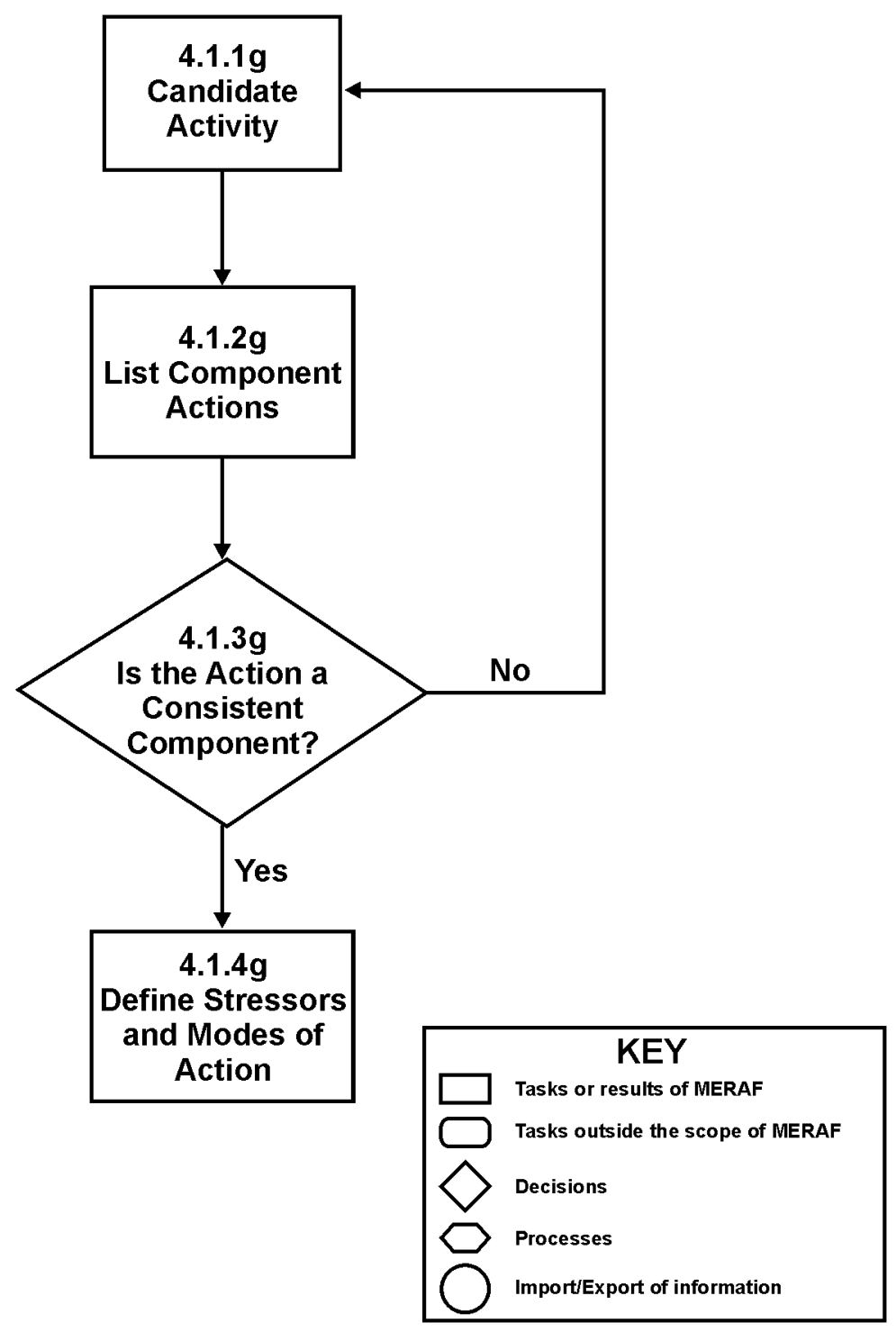

Chart 4.1g. Characterization of the Activity: Generic. 


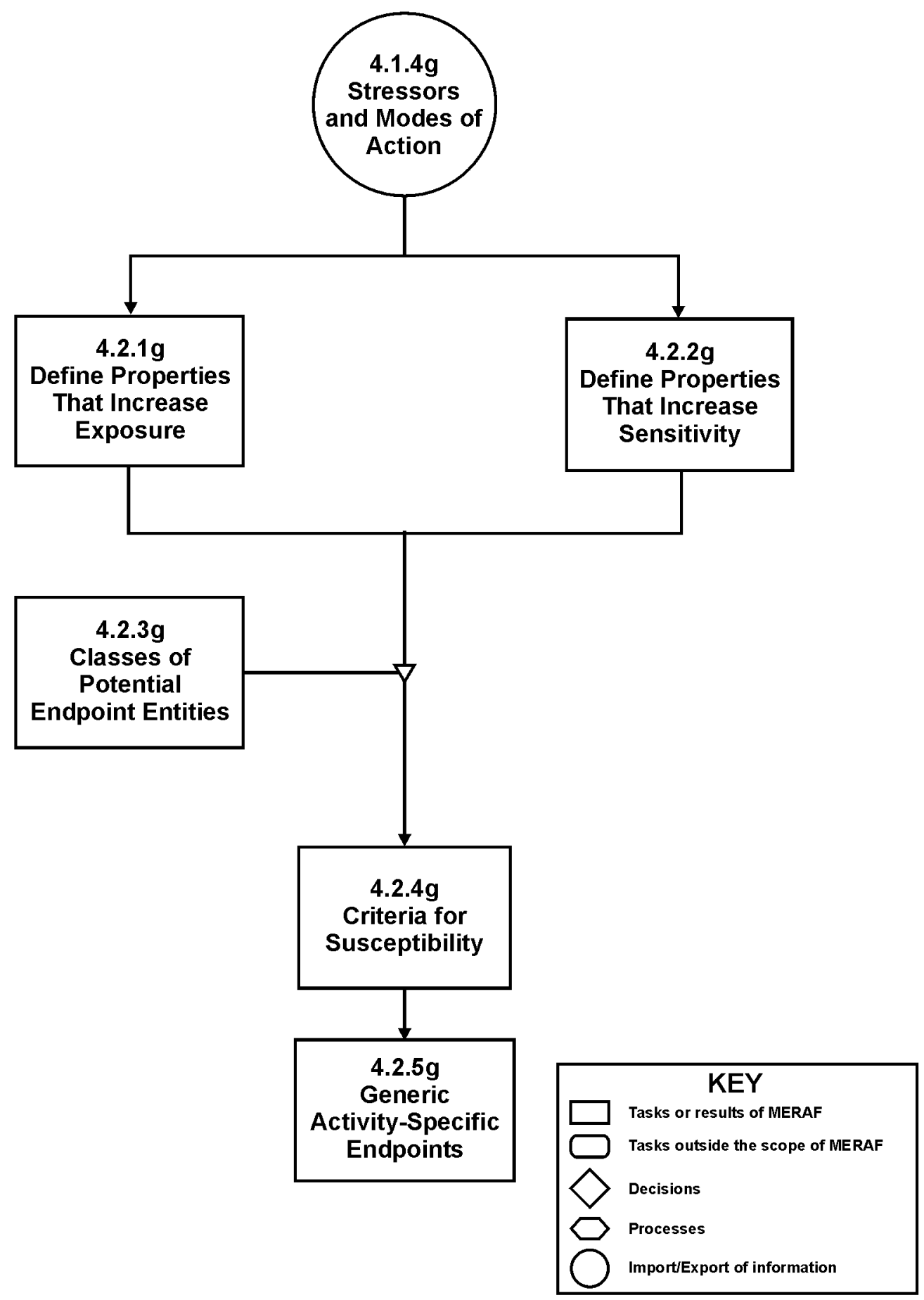

Chart 4.2g. Generic Activity-Specific Endpoint Selection. 


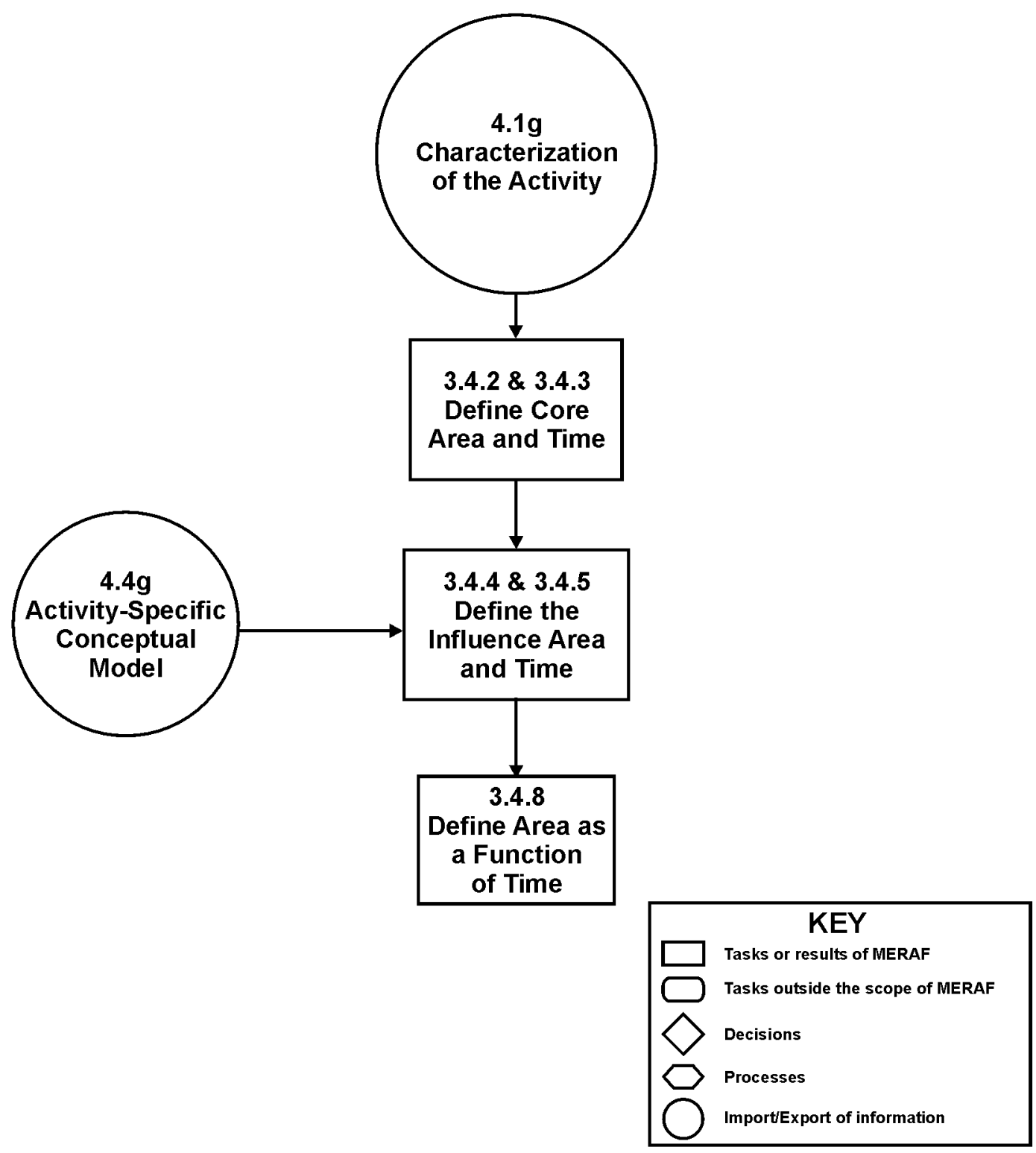

Chart 4.3g. Definition of Spatial and Temporal Extent. 


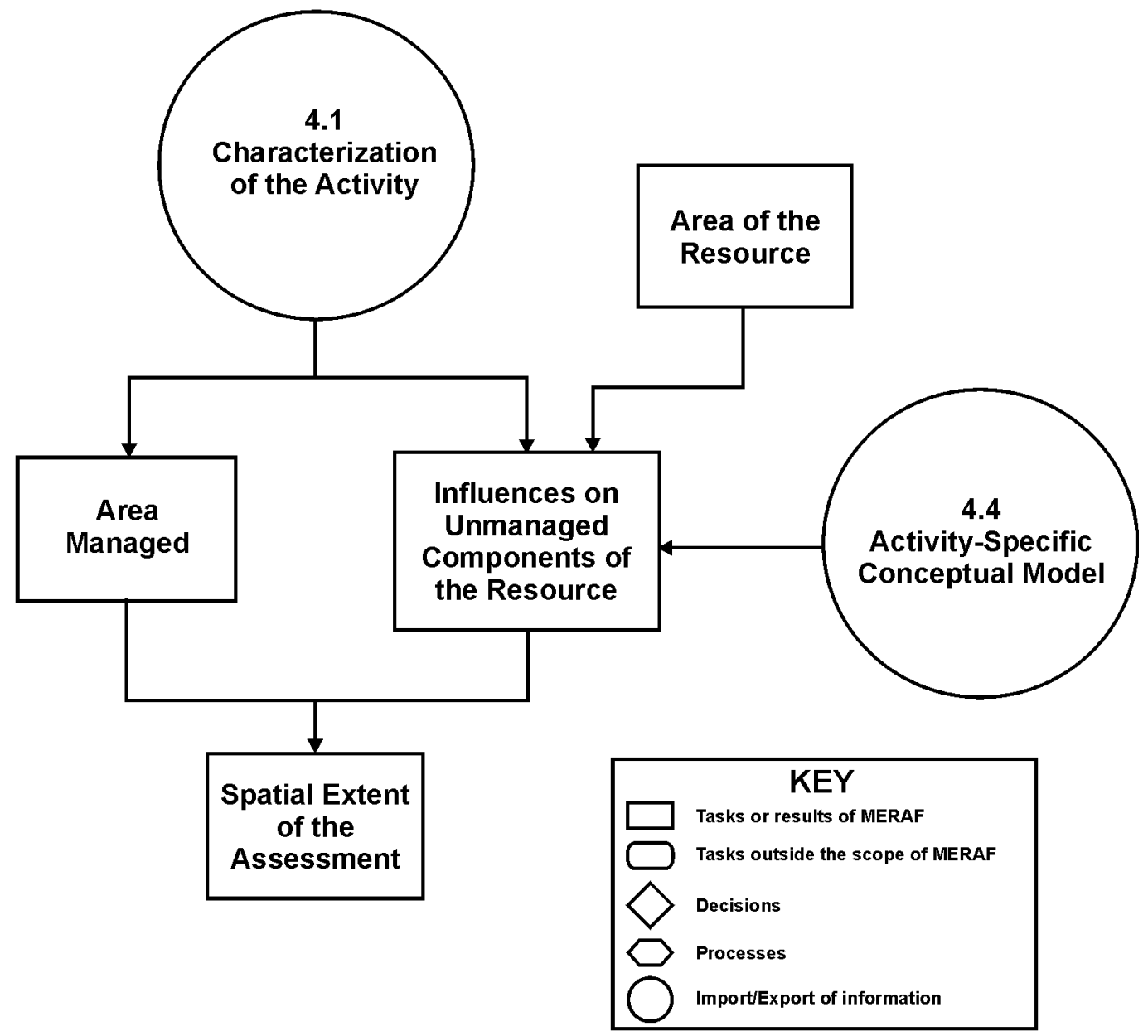

Chart 4.3r. Definition of Spatial Extent: Resource Management Activity. 


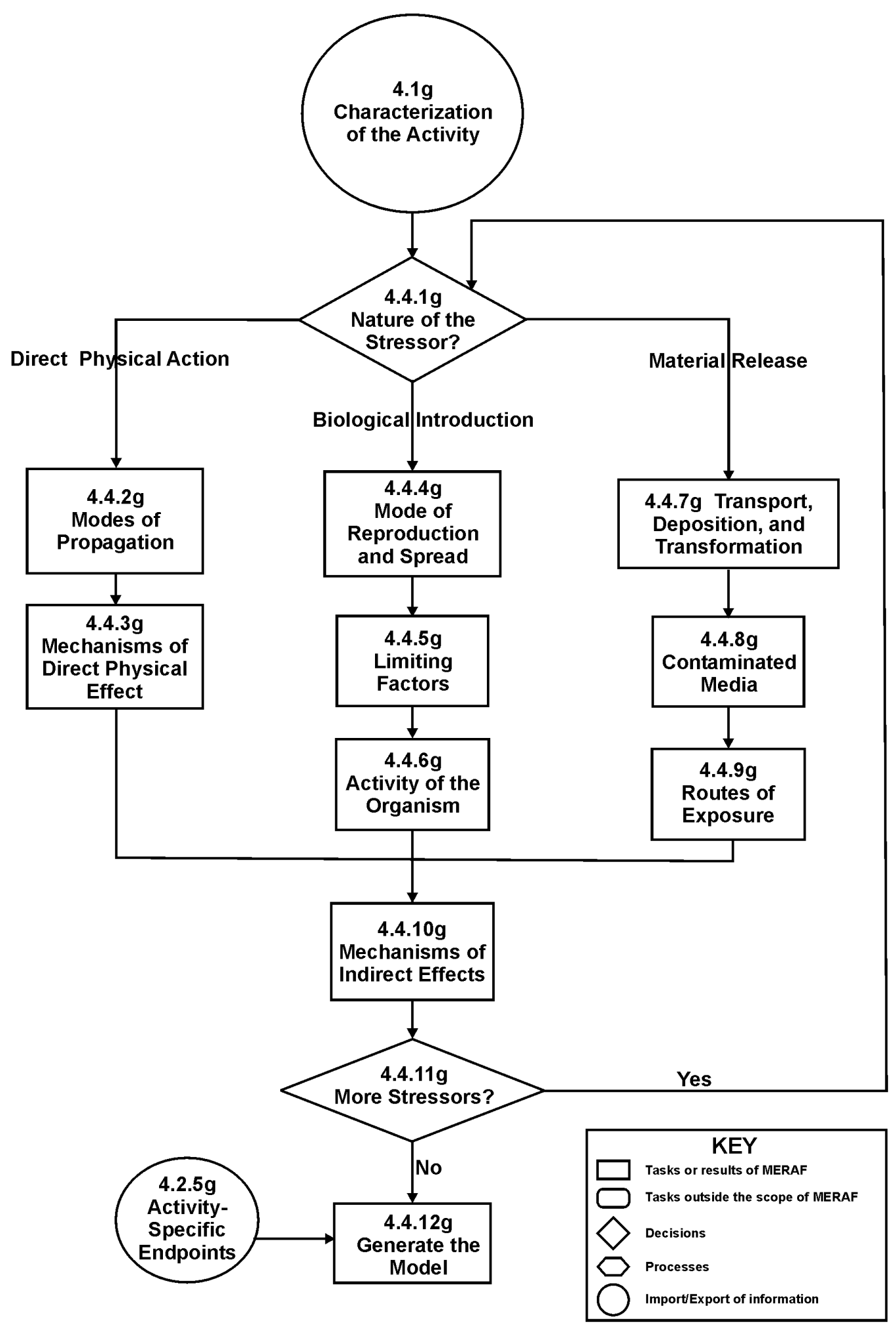

Chart 4.4g. Activity-Specific Conceptual Model: Generic. 


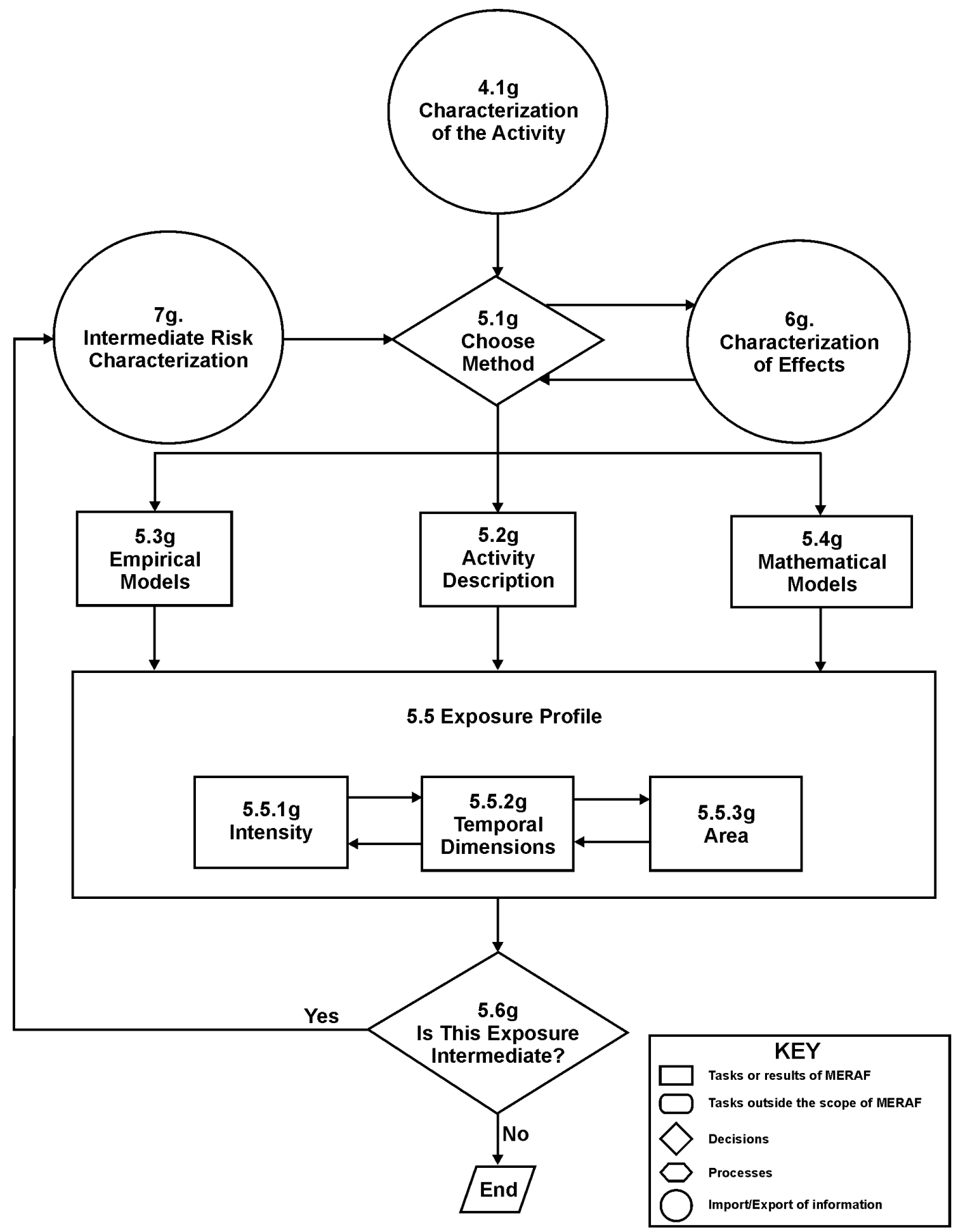

Chart 5g. Characterization of Exposure. 


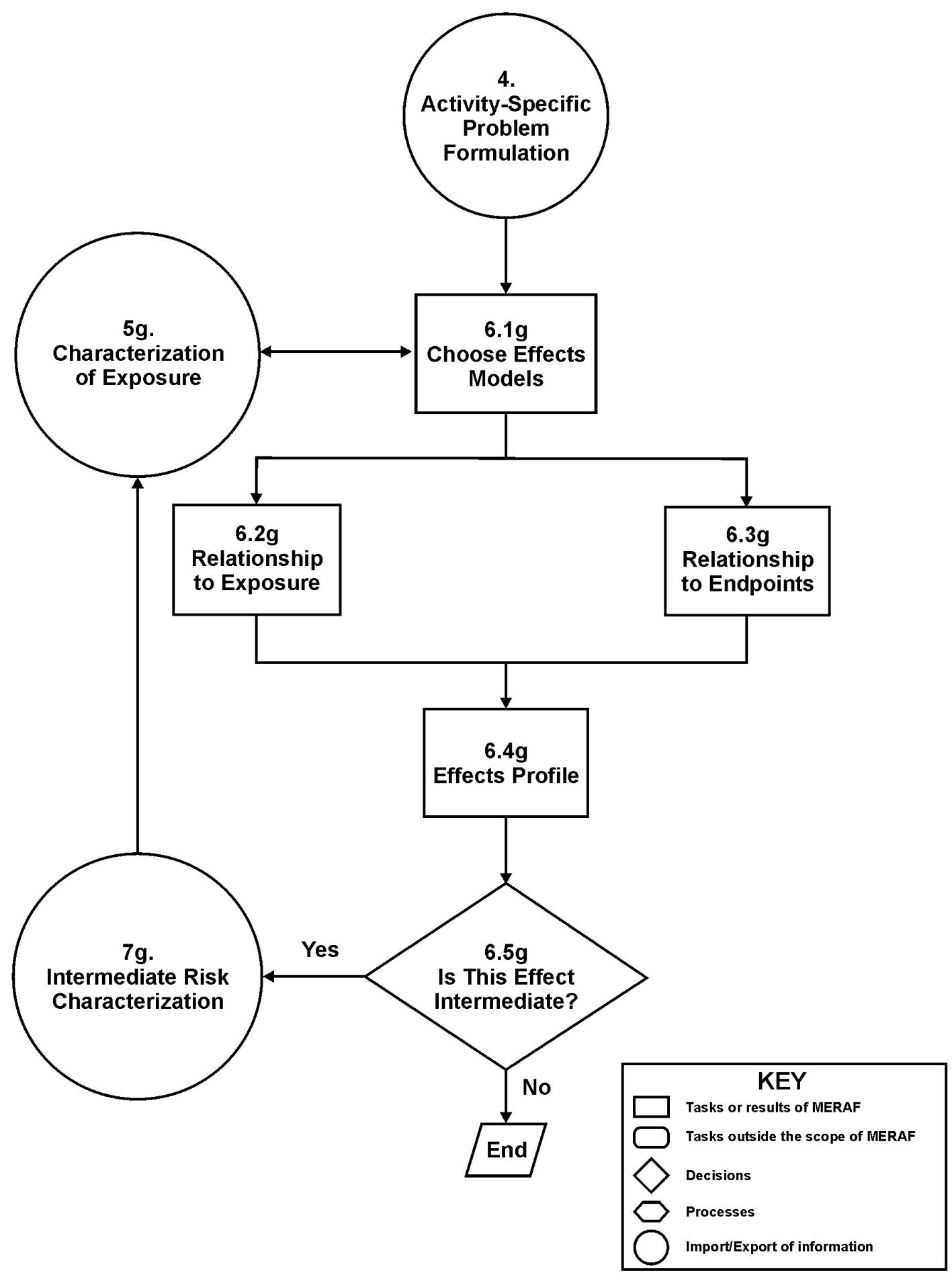

Chart 6g. Characterization of Effects. 


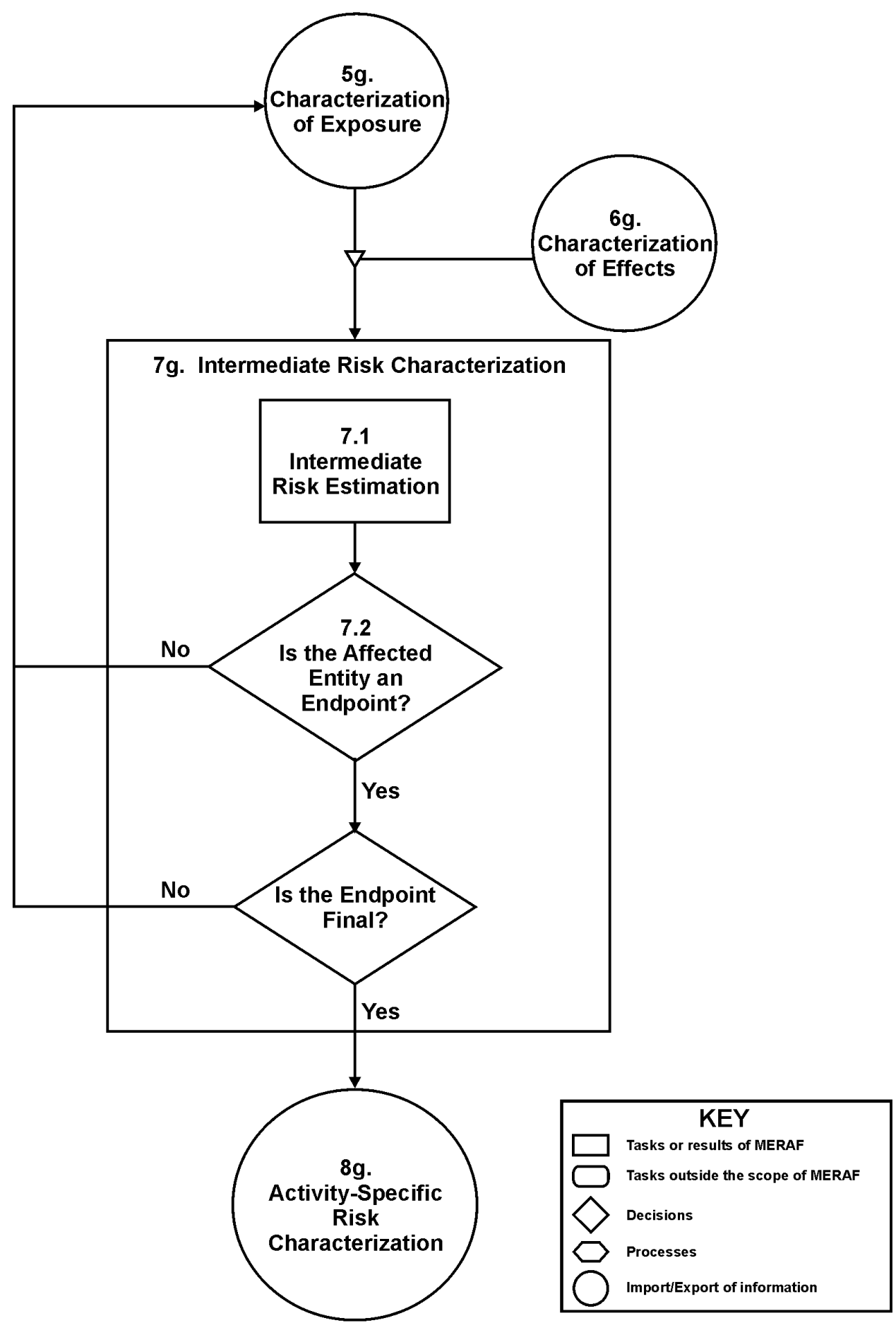

Chart 7g. Intermediate Risk Characterization. 


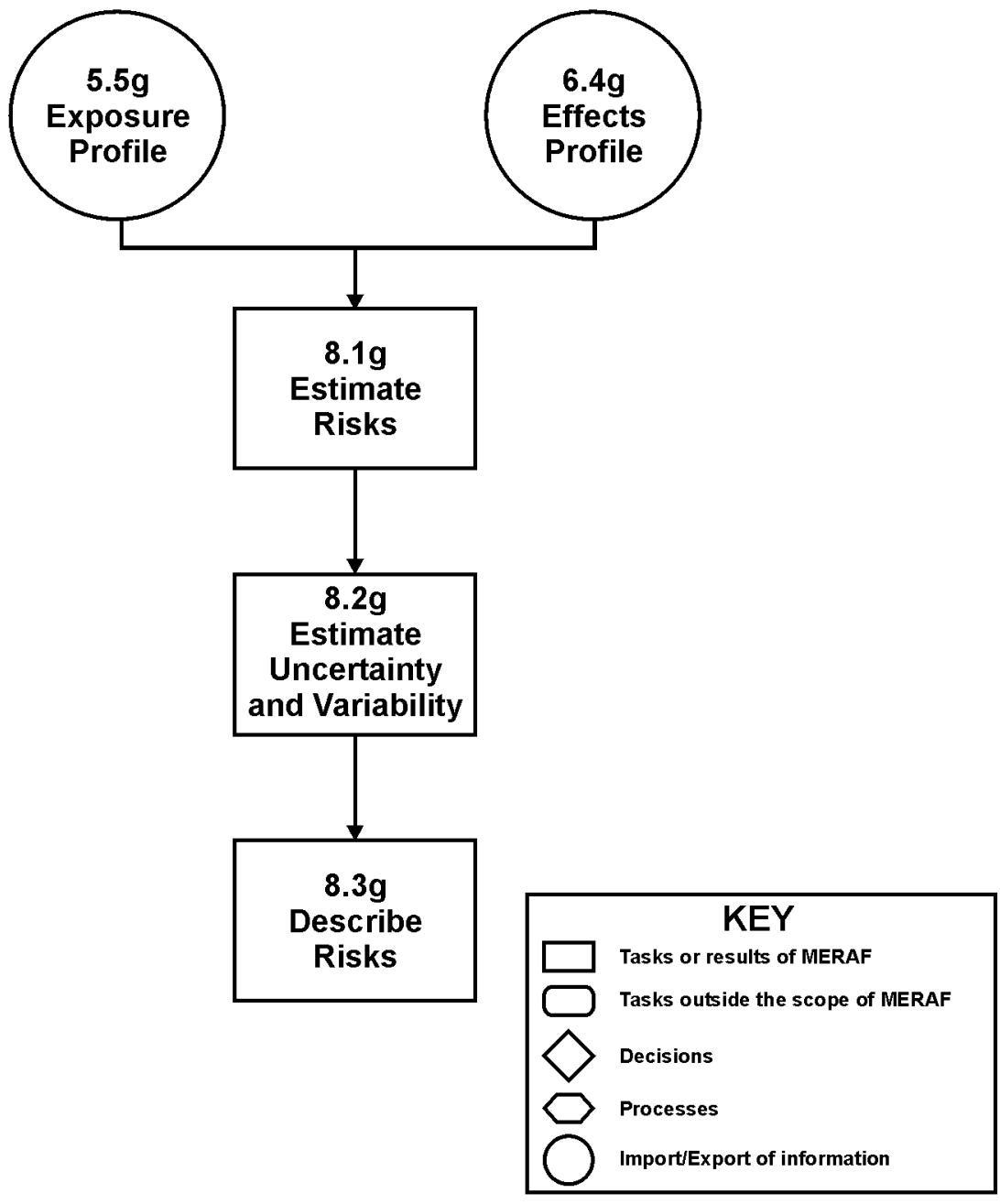

Chart 8g. Activity-Specific Risk Characterization. 


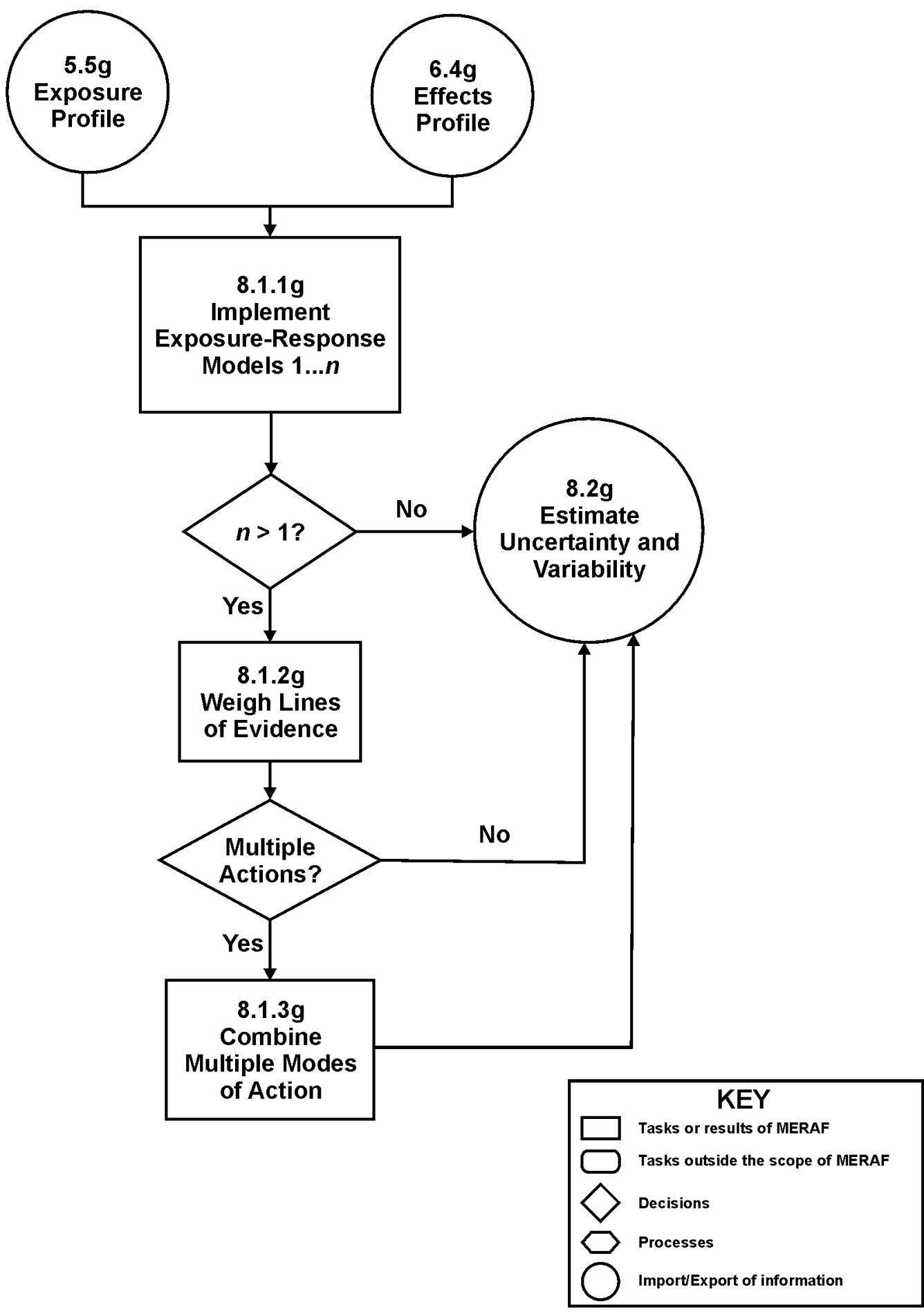

Chart 8.1g. Estimation of Risks. 


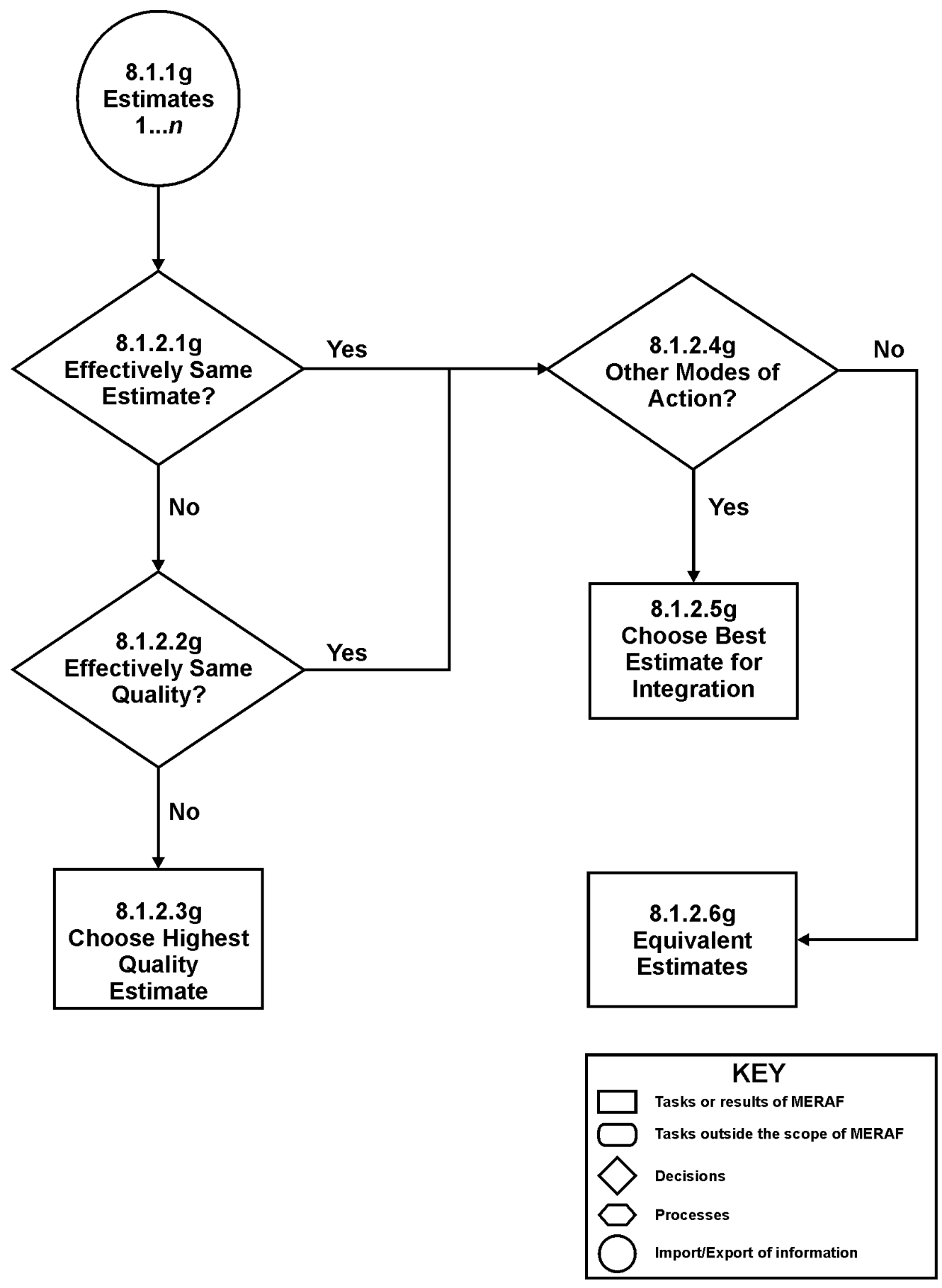

Chart 8.1.2g. Weight-of-Evidence. 


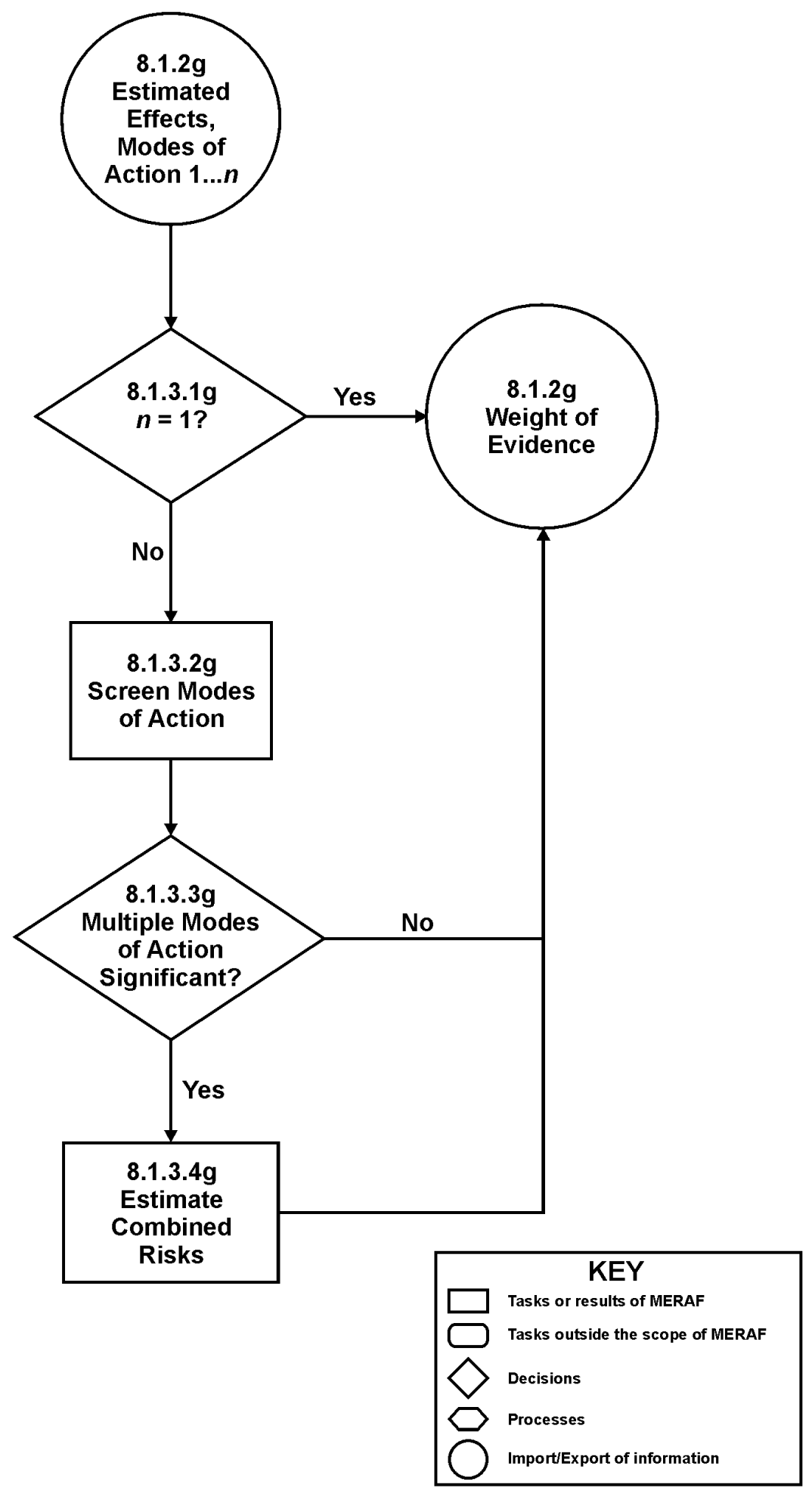

Chart 8.1.3g. Multiple Modes of Action. 


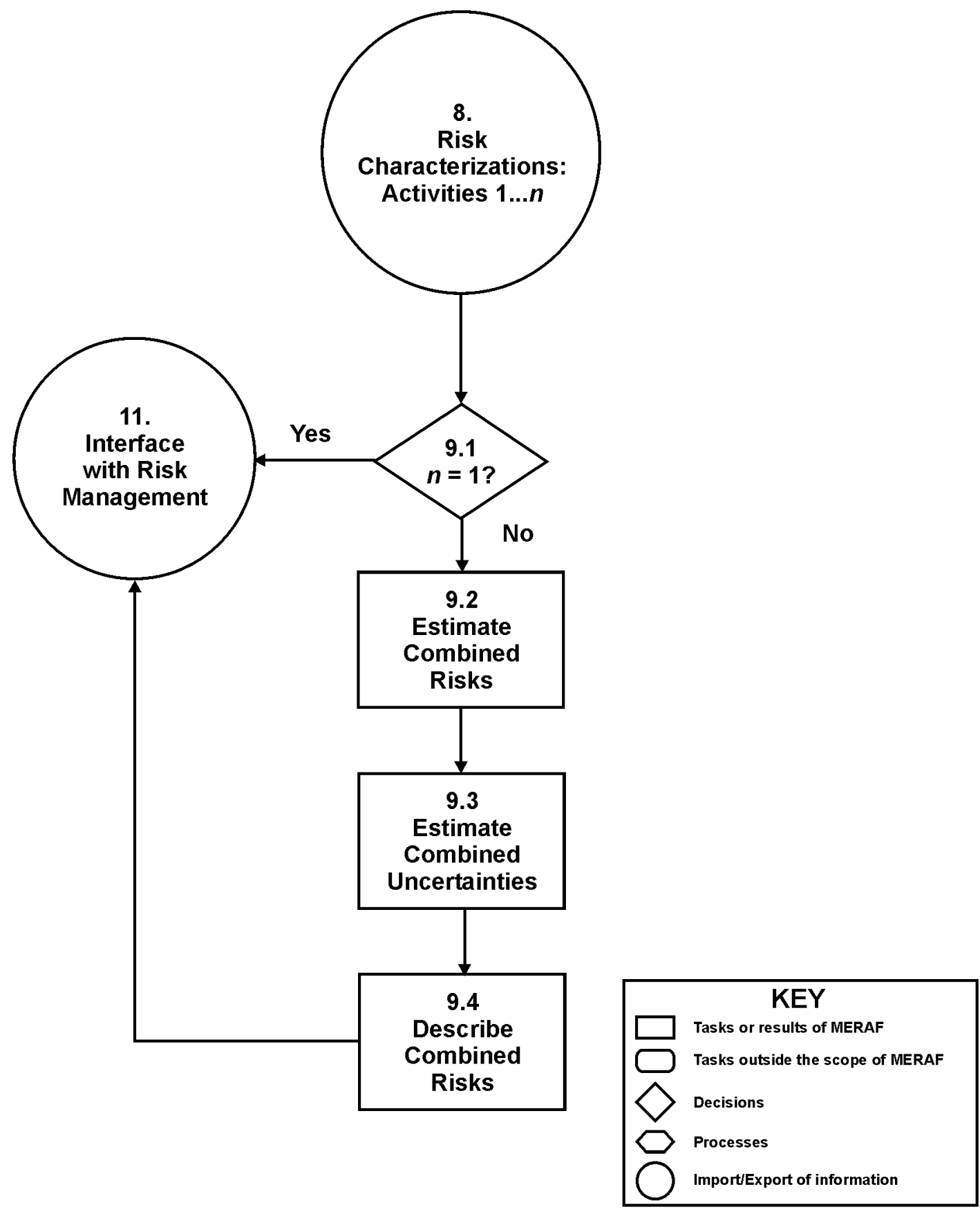

Chart 9. Integrated Risk Characterization. 


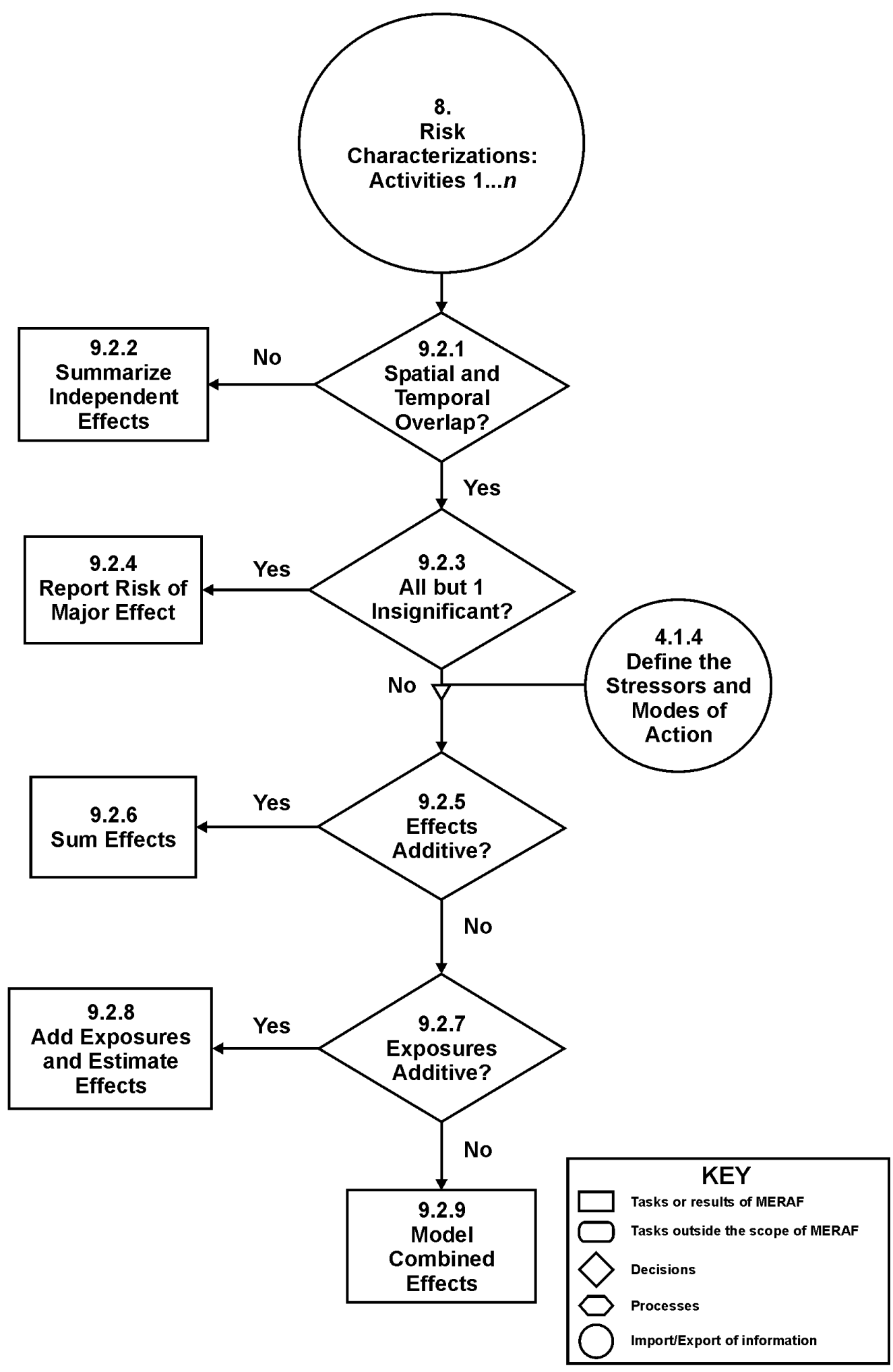

Chart 9.2. Estimate Combined Risk. 
ORNL/TM-2002/295

\section{INTERNAL DISTRIBUTION}

1. R. A. Efroymson

2. S. G. Hildebrand

3-5. ESD Library
6. ORNL Central Research Library

7. ORNL Laboratory Records-RC

\section{EXTERNAL DISTRIBUTION}

8. Dick Gebhart, Commander, ERDC-CERL, CN-C (Dick Gebhart, Ph.D.), P.O. Box 9005, Champaign, IL 61826-9005

9. Robert W. Holst, SERDP Program Office, 901 Stuart Street, Suite 303, Arlington, VA 22203

10. Glenn W. Suter II, National Center for Environmental Assessment, U.S. Environmental Protection Agency, MS 117, 26 Martin Luther King Drive, Cincinnati, OH 45268

11. Susan Walsh, SERDP Support Office, c/o HydroGeoLogic Inc., 1155 Herndon Parkway, Suite 900, Herndon, VA 20170 

